\title{
HetHetNets: \\ Heterogeneous Wireless Cellular Networks with Heterogeneous Traffic
}

\author{
by \\ Meisam Mirahsan, M.Sc. \\ A thesis submitted to the \\ Faculty of Graduate and Postdoctoral Affairs \\ in partial fulfillment of the requirements for the degree of
}

Doctor of Philosophy in Electrical and Computer Engineering
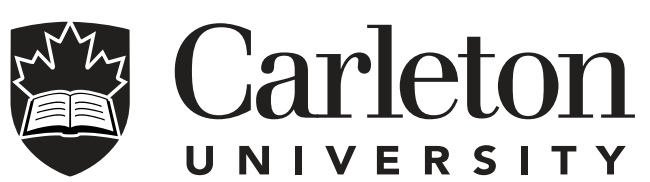

Ottawa-Carleton Institute for Electrical and Computer Engineering

Department of Systems and Computer Engineering

$$
\begin{gathered}
\text { Carleton University } \\
\text { Ottawa, Ontario }
\end{gathered}
$$

July, 2017

(C) Copyright

Meisam Mirahsan, 2017 


\section{Abstract}

One of the main expected characteristics of the envisioned $5 \mathrm{G}$ wireless cellular networks is heterogeneity. Heterogeneity is expected in both supply and demand. In the supply side, the network access part will be comprised of heterogeneous base stations (BSs) with different transmit powers, antenna heights, and radio technologies including macro-BSs, pico-BSs, femto-BSs, and wi-fi access points (HetNets). The spatial distribution of BSs is also heterogeneous (non-uniform) since the deployment of BSs is not carefully planned anymore and follows the customer requirements. In the demand side, the distribution of user equipments (UEs) is heterogeneous in the time domain as well as in the space domain due to the emergence of various applications with different rate requirements such as machine type communications (MTC) and also the heterogeneity of population density specially in municipal areas.

Nevertheless, an enormous majority of the existing literature on traffic modeling in wireless cellular networks consider only homogeneous (uniform) traffic scenarios. In particular, two independent Poisson point processes (PPPs) are excessively used to model the spatial distribution of UEs and BSs. PPP might be a fitting process for BSs but it is not an accurate model for the UE distributions. The assumption of independence between BSs and UEs is also not realistic since BSs (specially small-cell BSs) are usually deployed in UE hotspots.

In this thesis, we propose an accurate, realistic, simple, and adjustable modeling for the future heterogeneous wireless cellular networks with heterogeneous traffic distributions (HetHetNets). First, we propose a traffic modeling process describing a systematic approach to traffic modeling. According to the proposed process, we introduce a traffic modeling in which the heterogeneity of the UE distribution as well as the correlation between UEs and BSs are adjustable. Then, we show the impact of the traffic heterogeneity and the UE-BS correlation on the performance of HetHetNets. Finally, we present algorithms and applications in wireless networks which can exploit this realistic traffic modeling to enhance the network performance. 
I would like to dedicate my thesis to my lovely wife, Ghazaleh, who gave me the wings to fly, the courage to soar, and the reason to live. 


\section{Acknowledgments}

First and foremost, I am extremely grateful to my supervisor Prof. Halim Yanikomeroglu for his guidance, support, generosity, motivation and encouragement

during the past four years. Without his appreciation, I would not have had the chance to be a member of a prestigious research group that I have benefited so much from.

I am also extremely thankful to Dr. Rainer Schoenen for his consistent guidance and encouragement. He has spent so much effort on my research, and his broad knowledge has saved me from losing directions so many times.

Sincere thanks to all my colleagues for their support in both my research and my personal life.

On the personal side, I want to thank my family for their unconditional love. Without their support and faith in me, I would not be the man I am today. 


\section{Contents}

Abstract $\quad$ ii

Acknowledgments $\quad$ iv

Table of Contents $\quad$ v

List of Tables viii

List of Figures $\quad$ ix

Abbreviations $\quad$ xiv

Terms and Definitions $\quad$ xvi

1 Introduction 1

1.1 Motivation . . . . . . . . . . . . . . . . . . . 1

1.2 Literature Review . . . . . . . . . . . . . . . . . . . . . . 3

1.2.1 State of the Art on Heterogeneous Traffic Modeling . . . . . . 4

1.2.2 State of the Art on Heterogeneous Traffic Applications . . . . 5

1.3 Objective and Scope . . . . . . . . . . . . . . . . 8

1.4 Organization of Thesis . . . . . . . . . . . . . . . . . . . . . 9

1.5 Patents and Publications . . . . . . . . . . . . . . . . 10

2 The HetHetNet Framework 13

2.1 Introduction . . . . . . . . . . . . . . . . . . . . . . . 13

2.2 Traffic Modeling Methodology . . . . . . . . . . . . . . . . . . . 14

2.3 Traffic Measurement . . . . . . . . . . . . . . . . . 15

2.3.1 Traffic Measures . . . . . . . . . . . . . . 16

2.3.2 Traffic Statistics . . . . . . . . . . . . . . . . . . . . . . 19 
2.4 Heterogeneous Traffic Generation . . . . . . . . . . . . . . 24

2.4.1 The Basic Method . . . . . . . . . . . . . . . . . . . 24

2.4.2 The Enhanced Method . . . . . . . . . . . . . . . 25

2.5 Numerical Results . . . . . . . . . . . . . . . . . . . . . . . . . . . . . 29

2.5 .1 Traffic Modeling Results . . . . . . . . . . . . . . . . . 29

2.5.2 Network Performance Analysis Results . . . . . . . . . . 35

2.6 Conclusion . . . . . . . . . . . . . . . . . . . . . . . . . . . . . . . . . 39

3 Analytic Modeling of HetHetNets 40

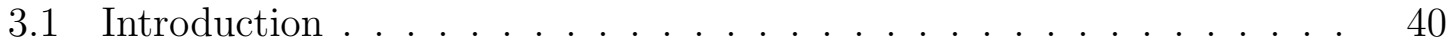

3.2 Proposed Non-Uniform UE Modeling . . . . . . . . . . . . . . . 41

3.3 Analytical Modeling . . . . . . . . . . . . . . . . . . 43

3.4 Simulation Results . . . . . . . . . . . . . . . . . . 46

3.5 Conclusion . . . . . . . . . . . . . . . . . . . 47

4 UIL in HetHetNets with Backhaul Capacity Constraints 49

4.1 Introduction . . . . . . . . . . . . . . . . . . . . . . . . 49

4.2 Modeling HetHetNets . . . . . . . . . . . . . . . . 54

4.2 .1 UE Distribution Model . . . . . . . . . . . . . . . . . . 55

$4.2 .2 \quad$ System Model . . . . . . . . . . . . . . . . . 57

4.3 Basic UIL . . . . . . . . . . . . . . . . . . . . . . . 57

4.4 Advanced UIL Schemes . . . . . . . . . . . . . . . . . . . 59

4.5 Simulation Results . . . . . . . . . . . . . . . . . . . 64

4.6 Conclusion . . . . . . . . . . . . . . . . . . . . . . . 65

5 VN Admission Control in HetHetNets 66

5.1 Introduction . . . . . . . . . . . . . . . . 66

5.2 System Model and Problem Definition _... . . . . . . . . . 69

5.2 .1 System Model . . . . . . . . . . . . . . . . . . . . . . 69

5.2 .2 Problem Definition . . . . . . . . . . . . . . . 71

5.3 Admission Control for Virtual Networks _ . . . . . . . . . . . . 73

5.3.1 Admission Control Process . . . . . . . . . . . . . . . . . 74

5.3 .2 Admission Control Method . . . . . . . . . . . . . . . . 75

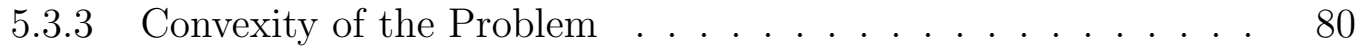

5.3 .4 Extensions . . . . . . . . . . . . . . . . . . 81 
5.4 Simulation Results . . . . . . . . . . . . . . . . . . 83

5.4.1 Simulation Setup and Parameters . . . . . . . . . . . . 83

5.4 .2 Simulation Results . . . . . . . . . . . . . . . . . 83

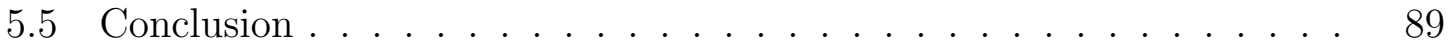

6 VNAC with Joint Backhaul and Access Optimization 92

6.1 Introduction . . . . . . . . . . . . . . . . . . . . 92

6.2 System Model and Problem Definition . . . . . . . . . . . . . . 96

6.3 Flow-Based VN Admission Control . . . . . . . . . . . . . . . . . 98

6.3.1 Flow Segmentation . . . . . . . . . . . . . . . . 98

6.3.2 Flow-Segment-Based VN Admission Control . . . . . . . . . . 99

6.4 Service-Based VN Admission Control . . . . . . . . . . . . . . . . 101

6.4.1 Service Segmentation . . . . . . . . . . . . . . . . . . 101

6.4.2 Service-Segment-Based VN Admission Control . . . . . . . . 101

6.5 Simulation Results . . . . . . . . . . . . . . . . . . . . . . 106

6.5.1 Simulation Setup . . . . . . . . . . . . . . . . 106

6.5.2 Complexity Comparison _. . . . . . . . . . . . . 107

6.6 Conclusion . . . . . . . . . . . . . . . . . . . . . . . . . . . . . 107

7 Conclusions and Future Work 108

7.1 Conclusions and Contributions . . . . . . . . . . . . . . . 108

7.2 Future Works . . . . . . . . . . . . . . . . . . . . . . . . 109

$\begin{array}{ll}\text { List of References } & 111\end{array}$

Appendix A Proof of the Simplest Polynomial Potential Function 121

Appendix B The Movement of the UEs to the New Locations 123

$\begin{array}{lll}\text { Appendix C Proof of Lemma 2 } & 125\end{array}$ 


\section{List of Tables}

3 Basic statistics of distance-based measures for a PPP in one, two and three dimensions and their analogues in time domain: $i$ is the process point index, $\lambda$ is the exponential distribution parameter for inter-arrival time and $\Lambda$ is the mean intensity of point processes. . . . . . . . . . 20

4 Simulation parameters for Chapter 2. . . . . . . . . . . . . 36

5 Simulation parameters for Chapter $4 \ldots \ldots$. . . . . . . . . 61

6 List of Symbols . . . . . . . . . . . . . . . . . . . . . 72

7 Simulation parameters for Chapter $5 \ldots \ldots$. . . . . . . 84

8 Multiple association versus max-SINR association . . . . . . . . . . 87

9 The impact of traffic spatial heterogeneity . . . . . . . . . . . . . 87

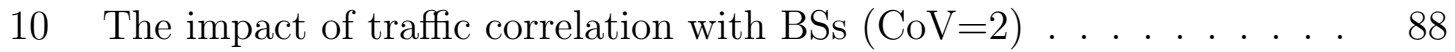

11 The impact of traffic correlation with $\mathrm{BSs}(\mathrm{CoV}=3) \ldots$. . . . . . 88

12 The impact of traffic correlation with $\mathrm{BSs}(\mathrm{CoV}=4) \ldots$. . . . . . . . 89

13 Comparison between flow-based and service-based methods in terms of number of variables and constraints. . . . . . . . . . . . . . . . 103 


\section{List of Figures}

1 Modeling procedure: The desired statistical properties of traffic as the modeling inputs are translated to the appropriate TGIPs, and traffic with known characteristics is generated to be used for network performance analysis (lower dashed box). The look-up table for translation is generated in advance via off-line calculations (upper dashed box). .

2 Voronoi and Delaunay tessellations: For a point pattern represented by bullets, the associated Voronoi tessellations (dashed lines) and Delaunay tessellations (solid lines) are illustrated. . . . . . . . . . . . . .

3 Domain similarity: Realizations of processes with sub-Poisson $(0<$ $C<1)$, Poisson $(C=1)$, and super-Poisson $(C>1)$ characteristics, respectively, from left to right in time domain (top) and space domain

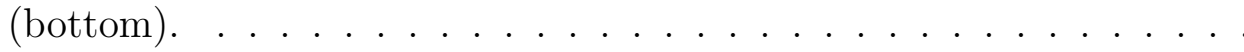

4 The weighted-Voronoi tessellation of BSs divides the whole field into cells associated with each BS consisting of all points closer, in terms of received signal power, to that BS than to any other BS. Every point in the field is then associated with a potential value between -1 and

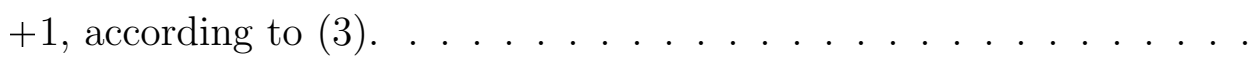

5 The $d(x, y)$ is the distance of the point $(x, y)$ to the associated cell center, and $D(x, y)$ is the length of the line connecting the associated cell center to the associated Voronoi cell edge through point $(x, y)$. . .

6 The CDF of the distribution of UEs in the Voronoi cell area of SAs: (a) When $\beta$ is deterministic, the UEs are moved towards SAs but the UE distribution shape is fixed and the Voronoi cell edge area of SAs remains empty with no UEs. (b) When $\beta$ is a random variable with normal distribution, even for high $\beta$ values, the Voronoi cell edges are not empty and there is a low probability for UEs existing at cell edges. 26

$7 \quad \beta$ is distributed with normal PDF with a mean value of $\mu_{\beta} \ldots \ldots$. . . 27 
8 Traffic distribution scenarios: By regulating only two parameters, $\alpha$ (the closeness indicator of social attractors to $\mathrm{BSs}$ ), and $\beta$ (the closeness indicator of UEs to social attractors) from 0 to 1 , traffic distributions with various properties ( $\mathrm{CoV}$ and BS-correlation) can be generated. The big circles denote macro-BSs, the triangles denote pico-BSs, the lines are the edges of the weighted-Voronoi tessellation of BSs, squares denote social attractors and small circles represent UEs. . . . . . . . .

9 To compare the proposed distance-based traffic measures with the existing nearest-neighbor distance measure, we fixed the $\alpha$ value to zero ( $\alpha=0$ ) and changed the $\beta$ value from 0 to 1 and calculated the $\mathrm{CoV}$ of different measures. Nearest-neighbor distance cannot capture the traffic heterogeneity and stays constant in super-Poisson region. . . .

10 To be consistent with time domain traffic measurement, we divide the $\mathrm{CoV}$ of the space traffic measures by the convergence value at Poisson patterns. The normalized CoV values are used in this thesis. . . . . .

11 The normalized CoV of Voronoi cell area is calculated for traffic gen-

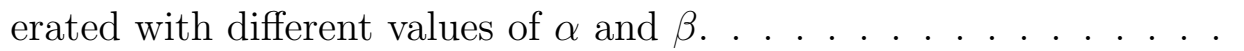

12 The correlation coefficient (refer to (9)) between UEs and BSs is calculated for traffic generated with different values of $\alpha$ and $\beta$. . . . .

13 The feasible normalized $\mathrm{CoV}$ values for different values of correlation coefficient are shown. Traffic with high correlation with BSs cannot have low normalized $\mathrm{CoV}$ values because high correlation means that UEs are gathered at cell centers. All the region above the solid line is the possible traffic generation using our proposed method. . . . . . .

14 The horizontal axis shows the desired normalized CoV. The desired normalized $\mathrm{CoV}$ is mapped to the associated $\alpha$ and $\beta$ values which are used to generate traffic. The vertical axis shows the calculated normalized $\mathrm{CoV}$ from the generated traffic patterns (for each desired $C$ value, the traffic is generated for all feasible $\rho$ values and the $\mathrm{CoV}$ is averaged out). . . . . . . . . . . . . . . . 
15 The horizontal axis shows the desired $\rho$. The desired $\rho$ is mapped to the associated $\alpha$ and $\beta$ values which are used to generate traffic. The vertical axis shows the calculated $\rho$ from the generated traffic patterns (for each desired $\rho$ value, the traffic is generated for all feasible normalized $\mathrm{CoV}$ values and the correlation coefficient is averaged out).

16 The mean user rates is shown for the feasible normalized $\mathrm{CoV}$ and correlation coefficient region. . . . . . . . . . . . . . . . . .

17 The coverage probability with minimum SINR threshold of $10 \mathrm{~dB}$ is shown for the feasible normalized $\mathrm{CoV}$ and correlation coefficient region. 38

18 Starting with a PPP, every UE is moved closer to its serving BS. . . . 42

19 The red squares denote the BSs and the blue lines represent their associated Voronoi tessellation. Small black dots denote the UEs. . . . 42

20 The CDF of SIR is illustrated. With increase in $n$ value, the distribution of SIR tends to higher values. . . . . . . . . . . . . . . . . 47

21 Mean UE SIR for different $n$ values is shown. . . . . . . . . . . . . 47

22 In the envisioned 5G HetHetNets, the assumption of backhaul connections with unlimited capacity is very optimistic. Specially, the envisioned femto-cells and pico-cells might have backhaul connections with constrained capacity. In this figure, a sample 3-tier HetHetNet with macro-cells, pico-cells and femto-cells, which have different backhaul capacities, is demonstrated. . . . . . . . . . . . .

23 Various UE distributions with different clustering properties are illustrated in a $1000 \mathrm{~m} \times 1000 \mathrm{~m}$ network. Black triangles denote the BSs and small dots denote the UEs. Starting from a pure Matern point process (a), the ratio of independent UEs increases and the $\mathrm{CoV}$ decreases until a homogeneous PPP (f) with no clusters is reached with $\mathrm{CoV}=1$. The number of BSs is 10 and the Matern cluster radius is assumed to be 50 meters. . . . . . . . . . . . . . . . . 
24 UIL aims at controlling user behavior in a network. User is guided to transmit at a better time or move to a better location. Based on the target behavior and the previous user behavior, the controller decides on the control information (CI) which is displayed in the screen of the user equipment for the user with the associated incentives (or penalties). The user decides 1) to follow the suggestion and deviate from his default behavior in order to receive the offered rewards, or 2) not to follow the suggestion and loose the offered rewards. . . . . . . . .

25 A fitting mathematical model is derived from the empirical probability distributions of using the offered services. The derived model is expressed as an exponential function parameterized by the offered moving distance and the associated incentives (or penalties). . . . . . . .

26 The mean user rate averaged over the entire network (left) and the coverage probability with minimum rate of $1 \mathrm{Mbps}$ (right) are shown in this figure (y-axis). The $\mathrm{x}$-axis shows the $\mathrm{CoV}$ values. Nonlinear least-squares fitting is used to show the curve trends. . . . . . . . .

27 Network structure: admission control, as a function in NOS, decides on admission or rejection of VN requests based on the customer traffic profiles, required QoE, and resource availability in SNs. . . . . . . . .

28 Admission control is performed in two levels: in the long-term level, VNs are admitted and embedded into the system statistically based on resource availabilities, and in the short-term level, single-sessions are admitted based on VN customer contracts. . . . . . . . . . . . . . . .

29 System model: VNs with heterogeneous traffic profiles to be served by a general SN with backhaul capacity limits. Every bin can be associated to one, two, or multiple BSs. . . . . . . . . . . . . . . . . . . . . 70

30 Specific input parameters and outputs of the admission control process are illustrated. . . . . . . . . . . . . . . . . .

31 VN admission control process: network-wide traffic measurement provides feedback information for the customers, traffic monitoring for QoE assurance, and possibility for an aggressive admission. . . . . . .

32 The downlink queues in BSs: every bin is served by multiple BSs with downlink queues which have different arrival rates and service rates which can be modeled by separate parallel M/M/1 queues. . . . . . . 
33 Analytical results versus simulation results: the small difference between the simulation and analytical results is in part due to the limited number of drops and shows the accuracy of the optimization results. .

34 A VN admission control method with multiple association is compared to single association and max-SINR association methods. Multiple association leads to significant increase in network performance hence VN admission control results. . . . . . . . . . . . . . . . . . . .

35 The figure shows the number of admitted VNs in backhaul limited scenarios. As expected, the number of admitted VNs goes down with decreasing the backhaul limits. . . . . . . . . . . . . . . .

36 Admission control process: admission decision is based on VN service requests and available resources. . . . . . . . . . . . . .

37 We consider a wireless cellular network with general access and backhaul topologies and SFC constraints. . . . . . . . . . . . . . . .

38 Flow segmentation: the original flow is parsed into smaller flow segments based on the SFC constraints. . . . . . . . . . . . . . . . . 98

39 Flow segments with common source node are categorized under a service segment. . . . . . . . . . . . . . . . . . . . . . . . 102

40 We consider a network with 2 PGWs, 4 SGWs, 7 BSs, and 21 sectors. 104

41 Computation time: the vertical axis shows the computation times for flow-based and service-based admission control algorithms. The horizontal axis shows the number of flows.

42 Computation time ratio: the ratio of computation time of flow-based admission control algorithm to the computation time of service-based algorithm is illustrated versus the number of flows. . . . . . . . . . . 106

43 Every BS Voronoi cell can be divided into sub-triangles which are each comprised of the BS as a vertex and the lines connecting the BS to the edge. . . . . . . . . . . . . . . . . . .

44 The potential value is assumed to be constant on each line parallel to the cell edges. So, the $\mathrm{P}$ value is only a function of $x$. . . . . . . . 


\section{Abbreviations}

$\begin{array}{ll}\text { 1D } & \text { 1 Dimensional } \\ \text { 2D } & 2 \text { Dimensional } \\ \text { 3D } & 3 \text { Dimensional } \\ \text { 3GPP } & \text { 3rd Generation Partnership Project } \\ \text { 5G } & \text { 5th Generation of Wireless Cellular Networks } \\ \text { AC } & \text { Admission Control } \\ \text { API } & \text { Application Programming Interface } \\ \text { BS } & \text { Base Station } \\ \text { CDF } & \text { Cumulative Distribution Function } \\ \text { CI } & \text { Control Information } \\ \text { CoV } & \text { Coefficient of Variation } \\ \text { C-RAN } & \text { Cloud-RAN } \\ \text { EMG } & \text { Exponentially Modified Gaussian } \\ \text { E-UTRA } & \text { Evolved Universal Terrestrial Radio Access } \\ \text { GPS } & \text { Global Positioning System } \\ \text { HCN } & \text { Heterogeneous Cellular Network } \\ \text { HetHetNet } & \text { HetNet with Heterogeneous Traffic } \\ \text { HetNet } & \text { Heterogeneous Network } \\ \text { HMM } & \text { Hidden Markov Model } \\ \text { IAT } & \text { Inter-Arrival Time } \\ \text { IoT } & \text { Internet of Things } \\ \text { IP } & \text { Internet Protocol } \\ \text { LTE } & \text { Long-Term Evolution } \\ & \end{array}$




$\begin{array}{ll}\text { MCF } & \text { Multi-Commodity Flow } \\ \text { MMPP } & \text { Markov Modulated Poissonian Process } \\ \text { MTC } & \text { Machine Type Communications } \\ \text { NFV } & \text { Network Function Virtualization } \\ \text { NOX } & \text { Network Operation System } \\ \text { OSI } & \text { Open Systems Interconnection } \\ \text { PDF } & \text { Probability Distribution Function } \\ \text { PPP } & \text { Poisson Point Process } \\ \text { QoE } & \text { Quality of Experience } \\ \text { QoS } & \text { Quality of Service } \\ \text { RAN } & \text { Radio Access Network } \\ \text { RB } & \text { Resource Block } \\ \text { SA } & \text { Social Attractor } \\ \text { SE } & \text { Spectral Efficiency } \\ \text { SFC } & \text { Service Function Chain } \\ \text { SFN } & \text { Service Function Node } \\ \text { SINR } & \text { Signal-to-Interference-plus-Noise Ratio } \\ \text { SIR } & \text { Signal-to-Interference Ratio } \\ \text { TG } & \text { Traffic Generator } \\ \text { TGIP } & \text { Traffic Generator Input Parameter } \\ \text { UE } & \text { User Equipment } \\ \text { UIL } & \text { User-In-the-Loop } \\ \text { VN } & \text { Virtual Network } \\ \text { VNAC } & \text { Virtual Network Admission Control } \\ \text { VNE } & \text { VN Embedding } \\ & \end{array}$




\title{
Terms and Definitions
}

\author{
a A typical bin \\ $b \quad$ A typical BS \\ C Coefficient of Variation (CoV) \\ d Traffic demand matrix \\ $l \quad$ A typical location \\ $p \quad$ Percentage of independent UEs \\ $r \quad$ Total received rate matrix \\ $t$ Time index \\ $W \quad$ Total bandwidth available at a BS \\ $x \quad$ Rate association matrix \\ A Set of bins \\ $B \quad$ Set of BSs \\ C Coverage requirement array \\ $D \quad$ Delay requirement array \\ $E$ Delaunay cell edge \\ $H \quad$ BS backhaul capacity array \\ $I \quad$ Inter-arrival time between points (events) \\ $K \quad$ Number of BS tiers \\ $L \quad$ Aggregate load on a BS \\ $M \quad$ BS loads array \\ $N_{a} \quad$ Number of users associated with a BS \\ $N_{t} \quad$ Number of points (events) arrived before time $t$ \\ $O \quad$ Outage requirement array
}




\begin{tabular}{ll}
$P$ & Potential function \\
$P_{N}$ & Noise power \\
$R$ & User target rates \\
$S$ & SA point pattern \\
$T_{i}$ & Time of arrival of point (event) $i$ \\
$U$ & User point pattern \\
$V$ & Voronoi cell area/volume \\
$W$ & BS available bandwidth array \\
$\alpha$ & Parameter for controlling closeness of SAs to BSs \\
$\beta$ & Parameter for controlling closeness of UEs to SAs \\
$\chi$ & Network cost \\
$\Delta$ & Average of experienced delay matrix \\
$\eta$ & SE matrix \\
$\Phi_{B}$ & BS generating point process \\
$\Phi_{U}$ & User generator point process \\
$\gamma$ & SINR matrix \\
$\Lambda$ & Mean intensity of point process \\
$\Theta$ & Experienced delay matrix \\
$\sigma$ & Mean value \\
$\mu$ & Standard deviation value \\
$\xi$ & Eorrelation between UEs and BSs \\
& Experienced coverage matrix \\
\hline &
\end{tabular}




\section{Chapter 1}

\section{Introduction}

\subsection{Motivation}

The 5th generation of wireless cellular networks $(5 \mathrm{G})$ are expected to be implemented and deployed in 2020s [1] to provide a high performance, high rate, and low latency radio access service to a diverse population of users with various user equipments (UEs) providing a reliable infrastructure for enabling advanced applications such as machine type communications (MTC) and Internet of things (IoT) [2].

Due to the rapid proliferation of a broad range of wireless devices such as smart phones and tablets with powerful processing capabilities and ambitious data rate expectations, the number of users relying on the wireless cellular infrastructure for Internet connectivity, as well as the traffic demand per user, are increasing dramatically while wireless resources remain limited. Getting the maximum performance gain out of these limited resources is the main focus of wireless cellular research.

With the advent of the increasingly diversified usage scenarios and applications in the envisioned $5 \mathrm{G}$ wireless networks, the traffic (demand) distribution in time domain and space domain is getting increasingly heterogeneous (non-uniform). Therefore, future generation (5G and beyond) cellular networks have to deal not only with an extreme traffic demand increase, but also an extreme level of heterogeneity in the distribution of that demand in both space and time.

Adjustable and realistic network traffic models in the space domain (i.e., UE distribution in the network) and the time domain (i.e., data rate demand for each UE over time) play a significant role in characterizing and analyzing the performance of a network as they provide traffic patterns to test network behavior under various conditions. As such, coping with the performance-related challenges in such networks 
often necessitates the availability of realistic, yet relatively simple and manageable, traffic models.

The statistics of the signal-to-interference-plus-noise ratio (SINR) are the key to the performance analysis of heterogeneous wireless cellular networks. The signal strengths and interference levels depend strongly on the network geometry, i.e., the relative positions of the transmitters and the receivers. Accordingly, in heterogeneous wireless cellular networks, spatial statistics of the traffic demand (UE distribution) as well as those of the service points (BS distribution) have direct impact on the network performance.

In the traditional single-tier homogeneous wireless cellular networks, usually, the macro-BSs have been assumed to be located in deterministic hexagonal grids [3]. With the advent of multi-tier heterogeneous cellular networks (HetNets) ${ }^{1}$, two major transitions have occurred in network modeling. First, small-cell BSs with varying communication characteristics (such as pico-BSs and femto-BSs) have been envisioned to be deployed in the network. Secondly, it has also become apparent that the regular grid topology assumption for BS locations does not hold anymore mainly due to the fact that small-cell BSs will often be deployed in UE hot-spots which are rather randomly distributed. Therefore, in the contemporary HetNets literature, BS locations have been modeled by Poisson point processes (PPPs).

In addition to the PPP model for the BS locations, it is becoming increasingly common to model the UE locations as another independent homogeneous PPP $[4,5]$.

Although the recently adopted PPP model for the UE locations is more realistic in comparison to the most common UE location model used in the earlier literature, in which the location of a fixed number of UEs in a cell are determined through two dimensional (2D) uniform random processes, the PPP model does not adequately represent the scenarios in which UEs are heterogeneously distributed (e.g., clustered). Moreover, the independence assumption between the two PPPs (for UE and BS locations) does not capture the correlation between the UE and BS locations observed in reality.

The real UE distributions are seldom pure PPPs. Due to the deployment of small-cell BSs in UE hot-spots, the correlation between UEs and BSs is an apparent phenomenon in HetNets. Network users are usually concentrated at social attractions such as residential and office buildings, shopping malls, and bus stations. Studying

\footnotetext{
${ }^{1}$ In this thesis we use the terms HetNet and Heterogeneous Cellular Network (HCN) interchangeably.
} 
the UE distributions of more extreme characteristics and their impact on network performance is thus an important investigation. The requirement is a continuously adjustable and tunable traffic model which can represent the broad possibilities from completely homogeneous cases (e.g., deterministic lattice) to extremely heterogeneous types (e.g., highly clustered scenarios), and from BS-independent UE locations to highly BS-correlated types.

Only when this realistic spatial traffic model is used in HetNet scenarios, the true impact of traffic characteristics on the network performance can be adequately captured. Obviously, the spatial UE distribution has a major impact on the network's key performance indicators, such as UE rates and outage probabilities which depend directly on UEs' SINR statistics. The spatial UE distribution has also an impact on the network energy efficiency. Especially in HetNets with dense small-cell deployments, the cell switch-off approach [6-8] is an effective scheme in energy saving; the extent of cell switch-off depends mainly on the distribution of UEs.

Many algorithms and applications in wireless cellular networks can be enhanced effectively knowing that the traffic is not homogeneous. For instance, user-in-the-loop (UIL) [9], which is a very effective method for shaping traffic distribution in wireless networks in the time domain and the space domain, is directly influenced by the heterogeneity of traffic demand.

On the other hand, many of the algorithms in wireless networks must be examined under heterogeneous scenarios to be considered as fully-tested and comprehensive solutions for network problems. One example of such algorithms is admission control. In admission control methods, the customer request to use the network services must be accepted or rejected. The admission control algorithm decides based on many parameters including the traffic demand distribution of the customer. Therefore, there must be a suitable traffic model for testing such algorithms which is able to model heterogeneous and BS-correlated traffic scenarios.

\subsection{Literature Review}

The literature review for this thesis can be divided into two main parts. The first part investigates the existing literature on the modeling of heterogeneous traffic in wireless networks. This part is presented in Section 1.2.1. The second part studies the current art on specific applications and algorithms in wireless networks which require 
heterogeneous traffic models. This part is explained in Section 1.2.2.

\subsubsection{State of the Art on Heterogeneous Traffic Modeling}

Traffic demand modeling in the time domain has been investigated well in the literature [10-17]. Traditionally in voice-only networks, homogeneous Poissonian models were accurate enough to model traffic in time. After the emergence of different applications, such as video and data with variable rates, the Poisson model failed to capture the traffic statistics [10]; as a result, various heterogeneous (super-Poisson) traffic models based on the hidden Markov model (HMM), Markov modulated Poisson process (MMPP) [11] and other stochastic methods have been proposed in the literature and used for performance analysis.

In the space domain, on the other hand, while there are many papers concentrating on the modeling of base station locations using stochastic geometry $[4,18]$, there are only few works in the literature which take into account the heterogeneous spatial distribution of traffic demand in wireless cellular networks [19-25]. To the best of the authors' knowledge, none of the existing works provides a statistically adjustable model representing a variety of possible scenarios for UE distribution.

In [19], Bettstetter et al., presented an algorithm to create a random inhomogeneous node distribution based on a neighborhood-dependent thinning approach in a homogeneous PPP. The model, however, can not be used for generating BS-correlated UE patterns, as this is beyond the scope of that model.

In [20], Qvarfordt and Legg presented non-uniform UE layouts (partly clustered around picocells) according to the $3 \mathrm{GPP}$ model 4a [21]. However, this model is not designed to adjust the traffic statistically, i.e., the traffic statistics are not measured to be used as an input to the traffic generation function.

Dhillon et al., in [22], proposed a non-uniform UE distribution model. They start with a higher density of BSs. Then they consider a typical UE located at the origin. After selecting the serving BS, they condition on this active link and independently thin the rest of the BS point process so that the resulting density matches the desired density of the actual BSs. It should be pointed out that the situations in which UEs are clustered, but not necessarily around BSs, are not captured by this method.

The spatial traffic modeling method proposed in [24], by Dongheon Lee et al., suggests that the spatial traffic can be approximated by the log-normal or Weibull distribution. This paper considers the statistics of the spatial traffic distribution, but 
it does not discuss the modeling of the cross-correlation of traffic with BS locations.

In [26] we proposed new measures for capturing traffic characteristics in the space domain. The proposed measures can be considered as the analogues of iat in the time domain. Thomas point process was used to generate spatial traffic patterns with desired characteristics. However, the HetNet scenarios are not investigated in our article [26].

In [27] we proposed a novel methodology for the statistical modeling of spatial traffic in wireless cellular networks. The proposed traffic modeling considered the cross-correlation between the UEs and BS locations as well as the CoV as defined in article [26]. The proposed traffic generation method was a density based method with two phases. First, a BS-biased non-uniform density function for the entire field was generated, then the desired point pattern was produced based on that density function. It should be noted, however, that the generation of the density function for all the points in the field (as required in the method proposed in [27]) is computationally intensive. Moreover, the model proposed in [27] is not directly applicable on HetNets because the density function is calculated for a homogeneous macro-only scenario.

In [28], we propose a superior traffic generation method in comparison to that in article [27] which is also applicable to HetNet scenarios and study the impact of spatially heterogeneous traffic on heterogeneous infrastructure. The proposed method is computationally efficient since it does not require the generation of a density function. Some of the key results in our previous works [26] and [27] are also presented in [28] in a comprehensive and coherent way with more analytical detail.

\subsubsection{State of the Art on Heterogeneous Traffic Applications}

After a short review of the current literature on heterogeneous traffic modeling, we need to study the applications and algorithms which are related to or affected by heterogeneous traffic.

The distributions of the wireless traffic demand and the wireless capacity supply don't necessarily match in the wireless cellular networks, neither in the time domain nor in the space domain. Commonly, some locations (times) of the network are overloaded while other locations (times) are underloaded. The problem with this lack of match is twofold: 1) In the overloaded parts of the network, the available resources must be shared to serve excessive amounts of demand, which leads to low data rates per user. 2) In the underloaded parts of the network, the unused resources 
are wasted. The root cause to this problem is that the idle network capacity and idle resources cannot be reserved in underloaded locations (times) to be transferred to the overloaded locations (times).

One solution to the problem of mismatch between supply distribution and demand distribution is to bring the wireless traffic demand and wireless capacity supply together in the time domain and the space domain. Predictive resource management and user location tracking [29], and optimized small-cell BS deployment in traffic hotspots, are among mechanisms which try to bring wireless capacity supply to wireless traffic demand location and time. On the other hand, user-in-the-loop (UIL) [9], defined below, is a relatively new and significantly effective method of bringing traffic demand to the time and location of wireless capacity supply (traffic shaping).

The UIL concept aims at influencing the user behavior (which can be viewed as the 'layer-8' in OSI network models) in a wireless network in order to obtain a better spectral efficiency by convincing the users to move from one location to a better one or to avoid traffic congestion by postponing session traffic out of the busy hours. Indeed, UIL extends the past assumption of the user being a traffic generating and consuming black box only (in nature similar to the noise input into a system). Instead, the system-theoretic framework allows a control input into the user block, on which the user receives suggestions and incentives (and eventually penalties) in order to convince him to diverge from the default behavior (which is uncontrolled, i.e., open loop), so that the traffic can be shaped [9].

Indeed, complementing the engineering for the growth of the supply side, the engineering for the control of the demand side is referred to as UIL and therefore motivated here. UIL proposes 'dynamic pricing' based on user behavior and his willingness to adapt with network situations, compared to current 'static pricing' policies. Results from a survey, which measures how willing a user is to respond to such control, are also presented in [9].

Another important algorithm in wireless networks which is dependent on the traffic statistics is admission control (AC). AC can be considered for single-session requests; for instance, when a single user submits a service request to the network. AC can also be considered for groups of user which submit a service request to the network as a group, i.e., virtual network (VN). In this thesis, we focus on $\mathrm{AC}$ for wireless virtual networks (VNs) in the context of network function virtualization (NFV). AC is a very well investigated concept and there is huge literature focusing on various 
aspects of AC [30-33]. However, since the idea of NFV is relatively new [34-36], AC for $\mathrm{VNs}$, also referred to as $\mathrm{VN}$ embedding (VNE), has been a hot research topic recently [37-40]. Indeed, AC for single users and single sessions is studied very well but the $\mathrm{AC}$ of groups of users (VNs) is relatively recent.

Although there is a rich literature on VNAC in wired networks, there are only few works on wireless VNAC [41-46]. In [41] and [42], even though the authors are discussing VNs, they consider fixed snapshots of UEs and statistical specification of traffic demand is not incorporated. In [43-46], the authors consider statistical arrival of VNs with statistical demands. However, the demand and the resources are not specified in rate or delay but in number of channels. To be more accurate, these papers assume that customers require a specific number of wireless channels, and the network allocates a number of channels to each UE upon availability, i.e., the spectral efficiency is not included in the allocation scheme and rate satisfaction is not the ultimate objective in these works.

The novelty and advantage of our proposed methods is that we consider customer satisfaction in terms of rate, packet delay, and outage probability at the same time which are important performance indicators and relevant QoE parameters for customers. We also assume that VNs have statistical demand profiles and the traffic density is specified for each VN and for various areas of the network.

In [31], a comprehensive classification of AC methods is provided. The first categorization is on central versus distributed methods. Even though the central methods, due to availability of more network-wide information, achieve higher efficiency, they are not appropriate for single-session AC since they are more complicated and result in long delays in decision making and users have lower patience thresholds. However, in VNAC, delay tolerance is higher and using centralized schemes is extremely beneficial. In this thesis, we propose a centralized $\mathrm{AC}$ scheme which takes place in NOX.

The next classification in [31] is single-class AC versus multiple-class AC. In traditional wireless networks, since the main network service was voice call, single-class AC schemes were beneficial. However, in 3G, 4G, and 5G+ networks, multimedia services with different QoE requirements are required. Therefore, multiple-class AC schemes are needed. The VNAC method proposed in this thesis is multiple-class and highly flexible in QoE specification.

Finally, the last classification is channel-quality-based versus rate-based methods. 
In the $\mathrm{AC}$ methods which are based on channel quality, the new customer is admitted if the channel quality is higher than their requirements. On the other hand, in ratebased AC methods, the new customers are admitted if their required rate can be provided. Although channel-quality-based methods are mathematically easier to deal with, they are not useful in future networks where BS load is a pivotal criteria and UE rates are totally dependent on BS load as well as channel quality. Our proposed method in this thesis is rate-based.

\subsection{Objective and Scope}

The main objective of this thesis is to present a novel heterogeneous spatial traffic modeling which allows statistical adjustment. Simple and non-parameterized, yet sufficiently accurate, measures for capturing the traffic characteristics in space are introduced. Only two statistical parameters related to the UE distribution, namely, the coefficient of variation (the normalized second-moment), of an appropriately defined inter-UE distance measure, and correlation coefficient (the normalized cross-moment) between UE and BS locations, are adjusted to control the degree of heterogeneity and the bias towards the BS locations, respectively. This model is used in heterogeneous wireless cellular networks (HetNets) to demonstrate the impact of heterogeneous and BS-correlated traffic on the network performance. This network is called HetHetNet since it has two types of heterogeneity: heterogeneity in the infrastructure (supply), and heterogeneity in the spatial traffic distribution (demand).

As a first step to fill the explained void in the literature in Section 1.2, the contributions of this thesis are summarized as follows:

- A spatial traffic modeling approach with adjustable statistical properties, capturing the severity of the heterogeneity and the extent of the correlation with $\mathrm{BSs}$, is introduced. Although the approach is presented in the context of heterogeneous wireless cellular networks, it is very flexible regarding the underlying network technology and it is general enough to be applied to other contexts as well, including Wi-Fi, ad-hoc, and sensor networks.

- Mathematical tools in stochastic geometry, including the Voronoi and Delaunay tessellations, are used to illustrate the similarities between traffic modeling in the time and space domains. This similarity leads to the introduction of new 
geometric inter-point distance measures for capturing the properties of spatial point patterns. These measures are chosen in such a way that they resemble the well-known inter-arrival time (iat) in the time domain.

- Only two parameters are introduced for a fairly accurate description of a heterogeneous and BS-correlated spatial traffic scenario:

1. The coefficient of variation $(\mathrm{CoV})$ values of the appropriately defined interpoint distance measures are used for specifying the deviations from homogeneity. As stated earlier, the discussed measures in the 2D space domain can be interpreted as the equivalents of the iat measure in the one dimensional (1D) time domain. It is worth mentioning that $\mathrm{CoV}$ is a commonly used statistic in traffic and queuing theories.

2. The correlation coefficient between the spatial UE distribution and the spatial BS distribution is used for specifying the bias of UEs towards BSs.

- The developed methodology is demonstrated in a heterogeneous wireless cellular network (HetNet) to illustrate the effects of the realistic traffic modeling on the performance.

- The heterogeneity of the traffic distribution is used in several algorithms and applications such as wireless virtual network admission control and user-in-theloop to enhance the network performance or reduce the network cost.

\subsection{Organization of Thesis}

This thesis is organized as follows. In Chapter 1, an introduction to the HetHetNets is presented and the motivations, existing literature, scope, and objectives of the thesis are explained along with the publications resulted from this line of research. The thesis is then divided into two main parts:

- In the first part (Chapters 2 and 3), various methods and aspects of HetHetNet modeling are studied. In Chapter 2, our novel methodology and procedure for HetHetNet modeling for future $5 \mathrm{G}$ networks is presented. We also introduce $\mathrm{CoV}$, a unified and non-parameterized metric for capturing and measuring the heterogeneity of UE distributions. An analytical investigation and modeling of HetHetNets is presented in Chapter 3. 
- In the second part (Chapters 4, 5, and 6), the algorithms and the applications which can take advantage of the proposed modeling to enhance network performance are described. In Chapter 4 the user-in-the-loop (UIL) method is investigated in a HetHetNet with limited backhaul capacities. In Chapter 5, the virtual network (VN) admission control problem is studied in a HetHetNet and is formulated as a convex optimization problem. In Chapter 6, a service-based admission control method is presented that includes the network backhaul in the optimization of VN admission control.

\subsection{Patents and Publications}

\section{Journal papers:}

1. Meisam Mirahsan, Rainer Schoenen, Halim Yanikomeroglu, Gamini Senarath, and Ngoc-Dung Dao, "User-in-the-loop for HetHetNets with backhaul capacity constraints", IEEE Wireless Communications, Special Issue on Smart Backhauling and Fronthauling for 5G Networks, vol. 22, no. 5, pp. 50-57, October 2015 [47].

2. Meisam Mirahsan, Rainer Schoenen, and Halim Yanikomeroglu, "HetHetNets: Heterogeneous traffic distribution in heterogeneous wireless cellular networks", IEEE Journal on Selected Areas in Communications, Special Issue on Recent Advances in Heterogeneous Cellular Networks, vol. 33, no. 10, pp. 2252-2265, October 2015 [28].

3. Meisam Mirahsan, Halim Yanikomeroglu, Gamini Senarath, and Ngoc Dao, "Analytic modeling of SIR in cellular networks with heterogeneous traffic", IEEE Communications Letters, vol. 20, no. 8, pp. 1627-1630, May 2016 $[48]$.

4. Meisam Mirahsan, Gamini Senarath, Ngoc Dao, and Halim Yanikomeroglu, "Admission control of wireless virtual networks in HetHetNets", under review in IEEE Transactions on Vehicular Technologies (submission: 22 October 2016, 1st results: 26 March 2017, 1st revision: 20 May 2017, 2nd results: 23 July 2017). 


\section{Conference papers:}

1. Meisam Mirahsan, Hamid Farmanbar, and Halim Yanikomeroglu, "Joint backhaul and access optimization for service-segment-based VN admission control", IEEE International Conference on Communications (ICC) 2017, 21-25 May 2017, Paris, France [49].

2. Meisam Mirahsan, Rainer Schoenen, Sebastian Szyszkowicz, and Halim Yanikomeroglu, "Spatial heterogeneity of users in wireless cellular networks based on open urban maps", IEEE International Conference on Communications (ICC) 2015, 8-12 June 2015, London, UK [50].

3. Meisam Mirahsan, Rainer Schoenen, and Halim Yanikomeroglu, "Statistical modeling of spatial traffic distribution with adjustable heterogeneity and BScorrelation in wireless cellular networks", IEEE Global Communications Conference (Globecom) 2014, 8-12 December 2014, Austin, TX, USA [27].

4. Meisam Mirahsan, Ziyang Wang, Rainer Schoenen, Halim Yanikomeroglu, and Marc St.Hilaire, "Unified and non-parameterized statistical modeling of temporal and spatial traffic heterogeneity in wireless cellular networks", IEEE International Conference on Communications (ICC) 2014 Workshop on 5G Technologies, 10-14 June 2014, Sydney, Australia [26].

\section{Patents:}

1. Method and apparatus for admission control of virtual networks in a backhaul-limited communication network [51]

Inventors: Meisam Mirahsan, Nimal Gamini Senarath, Ngoc Dung Dao, Hamidreza Farmanbar

Assignee: Huawei Technologies Co., Ltd.

(a) International application no: PCT/CN2016/101496, application date: Oct 8, 2016; publication no: WO2017059803 A1, publication date: Apr 13, 2017.

(b) US patent application no: 15/097,784, application date: Jun 14, 2016; publication no: US20170104688 A1, publication date: Apr 13, 2017. 
2. Methods and systems for provisioning a virtual network in software defined networks $[52]$

Inventors: Nimal Gamini Senarath, Ngoc-Dung DAO, Philippe Leroux, Meisam Mirahsan

Assignee: Huawei Technologies Co., Ltd.

(a) International application no: PCT/CN2015/091667, application date: Oct 10, 2015; publication no: WO2016055030 A1, publication date: Apr 14, 2016 .

(b) US patent application no: US 14/877,307, application date: Oct 7, 2015; publication no: US20160105893 A1, publication date: Apr 14, 2016. 


\section{Chapter 2}

\section{The HetHetNet Framework}

\section{$2.1 \quad$ Introduction}

A recent approach in modeling and analysis of the supply and demand in heterogeneous wireless cellular networks has been the use of two independent Poisson point processes (PPPs) for the locations of base stations (BSs) and user equipments (UEs). This popular approach has two major shortcomings. First, although the PPP model may be a fitting one for the BS locations, it is less adequate for the UE locations mainly due to the fact that the model is not adjustable (tunable) to represent the severity of the heterogeneity (non-uniformity) in the UE locations. Besides, the independence assumption between the two PPPs does not capture the often-observed correlation between the UE and BS locations.

This chapter presents a novel heterogeneous spatial traffic modeling which allows statistical adjustment. Simple and non-parameterized, yet sufficiently accurate, measures for capturing the traffic characteristics in space are introduced. Only two statistical parameters related to the UE distribution, namely, the coefficient of variation (the normalized second-moment), of an appropriately defined inter-UE distance measure, and correlation coefficient (the normalized cross-moment) between UE and BS locations, are adjusted to control the degree of heterogeneity and the bias towards the BS locations, respectively. This model is used in heterogeneous wireless cellular networks (HetNets) to demonstrate the impact of heterogeneous and BS-correlated traffic on the network performance. This network is called HetHetNet since it has two types of heterogeneity: heterogeneity in the infrastructure (supply), and heterogeneity in the spatial traffic distribution (demand). 


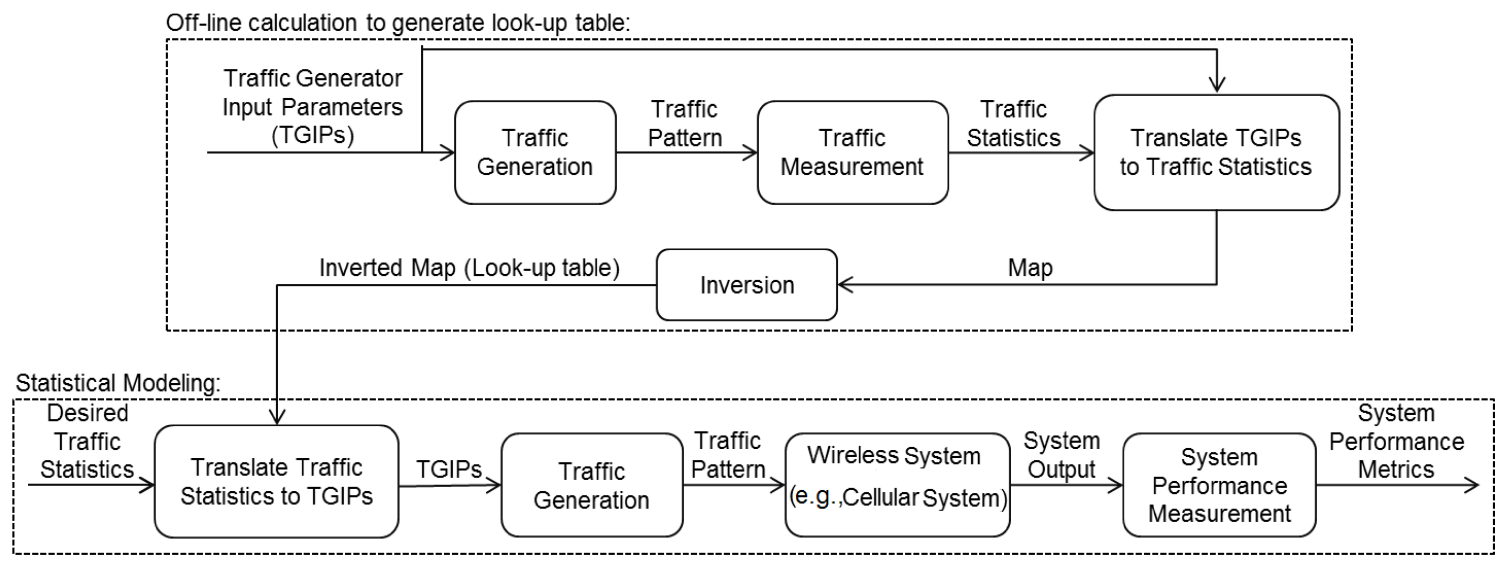

Figure 1: Modeling procedure: The desired statistical properties of traffic as the modeling inputs are translated to the appropriate TGIPs, and traffic with known characteristics is generated to be used for network performance analysis (lower dashed box). The look-up table for translation is generated in advance via off-line calculations (upper dashed box).

\subsection{Traffic Modeling Methodology}

Heterogeneous wireless cellular network models receive traffic patterns in the time domain as their input for performance analysis. The key performance indicators such as, average user data rates, system spectral efficiency, and outage probability, are calculated as the system outputs. The traffic patterns are usually generated by random processes (e.g., PPP, MMPP, HMM, ...), which are called traffic generators (TGs).

TGs, in turn, receive a number of input parameters (TGIPs) to generate traffic patterns with various statistical properties. Therefore, a main track of research in traffic modeling has been to fit TGIPs to generate traffic patterns with the desired statistical properties (e.g., measured statistics from a real traffic trace) $[17,53,54]$.

Figure 1 demonstrates our proposed traffic modeling methodology in the space domain. A somewhat similar methodology has been used in the literature for traffic modeling in the time domain [17].

The proposed methodology is described as follows:

- The desired statistical properties of traffic as the modeling inputs are fed to the traffic generator. 
- Since the traffic generator expects TGIPs, the desired statistics are first translated to the appropriate TGIPs.

- Traffic with known characteristics is then generated to be used for network performance analysis.

- The translation look-up table is developed off-line in advance:

- The entire range of the feasible TGIP values are fed to the traffic generator and the traffic pattern statistics are measured at the output. Therefore, a complete map from TGIP values to the corresponding traffic statistics is produced.

- The conversion function from TGIPs to the traffic statistics is obtained by fitting methods.

- The look-up table from traffic statistics to the corresponding TGIPs can be prepared by inversion of the map or the function from TGIPs to the measured statistical properties.

A mathematical description of the process is given in Section 2.4.2.

The method described above is capable of generating a wide range of heterogeneity possibilities. However, an appropriate next-step is to tune the model according to the real world measurements and to extract the accurate model parameters for generating realistic traffic patterns. In [50], we used the maps of Paris, France, obtained from OpenStreetMaps [55], to study the spatial traffic heterogeneity of outdoor users in the denser areas of the city center.

\subsection{Traffic Measurement}

A perfect model will require all the statistics of the traffic measures for the generated traffic patterns and those for the real traffic traces, including the cumulative distribution functions (CDFs) and auto-correlation functions, to match. Therefore, a perfect match is not practical as this requires the use of extremely complicated models with a very large number of parameters. As a result, simplified models are commonly used which only consider the first few moments of the traffic. In the time domain, usually the mean, $\mathrm{CoV}$, and auto-correlation, and rarely the third moment, are considered to match with those of the real traffic trace. 
For the measurement of traffic patterns, first, an appropriate measure must be selected to capture the traffic properties. In the time domain, iat is the most popular and well accepted measure.

In Section 2.3.1, the equivalent measures for the statistics of the spatial traffic are introduced. The spatial traffic statistics are described in Section 2.3.2.

\subsubsection{Traffic Measures}

Packet arrivals in the time domain can be modeled by a one-dimensional (1D) point process. A fixed iat between packets generates the maximum homogeneity (deterministic lattice). An exponentially distributed iat generates complete randomness (PPP in 1D). For generating sub-Poisson patterns (patterns with more homogeneity than Poisson), one way is to generate a perfect lattice, then apply a random displacement (perturbation) on its points $[56,57]$. Various models for generating super-Poisson patterns (patterns with more heterogeneity than Poisson) have been proposed in the literature which are mostly based on hierarchical randomness and Markov models $[10,15,17]$.

A $1 D$ point pattern in the time domain can be measured mathematically in many different ways. One may use the interval count, $N(a, b]=N_{b}-N_{a}\left(N_{t}\right.$ representing the number of points arrived before time $t$ ), which is a density-based measure and divides the whole domain into smaller windows and counts the number of pattern points in each window. A disadvantage of the density-based measures is that they are parameterized by the window size. Finding an appropriate window size is itself a challenging question and does not have a unique answer for all applications.

The inter-arrival time, iat, $I_{i}=T_{i+1}-T_{i}\left(T_{i}\right.$ representing the time of arrival of point $i$ ), is the most popular and best accepted measure because it is distancebased rather than density-based, and it considers the distance between every two neighboring points in the domain. The $\mathrm{CoV}$ for iats is defined as

$$
C_{I}=\frac{\sigma_{I}}{\mu_{I}}
$$

where $\mu_{I}>0$ and $\sigma_{I}$ are the mean and standard deviation of iats, respectively (note that $\mu_{I}>0$ if $\left.t>0\right)$. For a perfect $1 \mathrm{D}$ lattice, the constant iat has $C_{I}=0$. For a $1 \mathrm{D}$ Poisson pattern, $C_{I}=1$, since for an exponential distribution with parameter $\lambda$, the standard deviation and the mean are both $\mu_{I}=\sigma_{I}=\lambda$. Sub-Poisson processes have 
$0<C_{I}<1$ and super-Poisson processes have $C_{I}>1$.

The UE locations in a heterogeneous wireless cellular network in space domain can be modeled by a two-dimensional (2D) or three-dimensional (3D) point process. A very inclusive review of Point processes in space domain is conducted in [58]. A fixed distance between points generates perfect homogeneity (deterministic lattice). On the contrary, the Poisson distribution generates complete randomness. For generating sub-Poisson patterns, one way is to generate a perfect lattice, then apply a random perturbation on its points [56-58].

As mentioned above, in time domain, the distance-based measure iat captures heterogeneity by one non-parameterized real value $C_{I}$. In multi dimensions, however, there is no natural ordering of the points, so finding the analogue of the iat is not straightforward. There are many density-based heterogeneity measures in the literature such as Ripley's K-function and pair correlation function [58], but they are all parameterized. For introducing distance-based measures, there is the problem of defining the 'next point' or the 'neighboring points' in multi-dimensional domains.

The first and simplest candidate for characterizing a neighboring point in a multidimensional domain is to consider the nearest-neighbor. This leads to the nearestneighbor distance measure [59]. However, the nearest-neighbor distance measure in $1 \mathrm{D}$ time domain is not the analogue of the $i a t$ because it considers the $\min \left\{I_{i}, I_{i+1}\right\}$ for every point $T_{i}$. It is shown in Section 2.5 in our simulation results that the nearestneighbor distance fails to capture the process statistics in multi-dimensional domains because this measure ignores the neighbors other than the closest one. The next candidate is the distance to the $k^{\text {th }}$ neighbor. However, determining $k$ globally is not possible because every point may have a different number of neighbors.

In the following, novel distance-based and non-parameterized measures in the space domain are proposed based on the Voronoi and Delaunay tessellations.

Definition 1. Voronoi Tessellation [60, p. 1]: Given a point pattern $P=$ $\left\{p_{1}, p_{2}, \ldots, p_{n}\right\}$ in $d$-dimensional space $\mathbb{R}^{d}$, the Voronoi tessellation $V T=$ $\left\{c_{p_{1}}, c_{p_{2}}, \ldots, c_{p_{n}}\right\}$ is the set of cells such that every location, $y \in c_{p_{i}}$, is closer to $p_{i}$ than any other point in $P$. This can be expressed formally as

$$
c_{p_{i}}=\left\{y \in \mathbb{R}^{d}:\left|y-p_{i}\right| \leqslant\left|y-p_{j}\right| \text { for } i, j \in 1, \ldots, n\right\}
$$

Definition 2. Delaunay Tessellation [61, p. 11]: The Voronoi tessellation in $\mathbb{R}^{d}$ has 


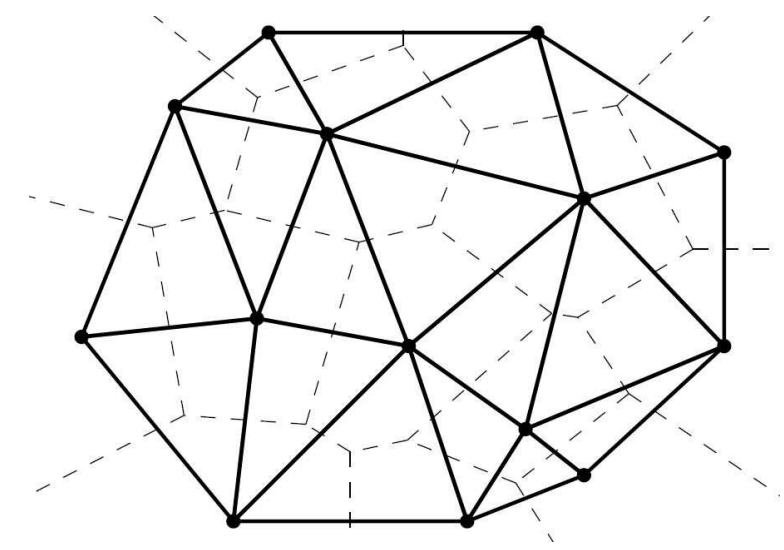

Figure 2: Voronoi and Delaunay tessellations: For a point pattern represented by bullets, the associated Voronoi tessellations (dashed lines) and Delaunay tessellations (solid lines) are illustrated.

the property that each of its vertices is given by the intersection of exactly $d+1$ Voronoi cells. The corresponding $d+1$ points define a Delaunay cell. So the two tessellations are said to be dual.

Figure 2 demonstrates a pattern of points with its Voronoi tessellation (dashed lines) and Delaunay tessellations (solid lines).

Definition 3. Natural Neighbor [62, p. 3]: Every two points sharing a common edge in Voronoi tessellation or equivalently every two connected points in Delaunay tessellation of a point process are called 'natural neighbors'.

Natural neighborship gives an inspiration of neighboring relation in multidimensional domains and leads us to the analogues of the well accepted iat measure in multi dimensions. Various statistical inferences based on different properties of cells generated by these tessellations can be considered for the measurement of a point pattern.

The Voronoi cell area or Voronoi cell volume $V$ is the first natural choice. For a lattice process, all the cell areas in 2D or cell volumes in 3D are equal which results in $C_{V}=0$. The statistics of the Voronoi cells for a PPP (Poisson-Voronoi tessellation) are well investigated in the literature [60, 63-66]. Square-rooted Voronoi cell area in 2D or cube-rooted Voronoi cell volume in 3D can also be considered. We are interested in Voronoi cell area $V$ because it can be considered as an analogue of iat in the time domain. 
The main focus of this thesis is the spatial aspect of traffic distribution because the temporal aspect is well-investigated in the literature. The combination of the temporal domain and the spatial domain, which is a very challenging problem, can be considered as an extension of this thesis. However, it is critical to understand the heterogeneity in the space domain thoroughly before proceeding to the combined domain. In that case, some other metrics such as the inverted Voronoi cell area, $1 / V$, could be used to normalize the user target rates in the time domain. Therefore, a combined metric such as $R / V$ can be of interest in the combined domains; this remains as an interesting future work.

The next proposed measure is the Delaunay edge length $E$. The statistics of Delaunay tessellations is investigated in [67-69]. The mean value of the lengths of Delaunay edges of every point can also be considered.

A Delaunay tessellation divides the space into triangles or tetrahedrons in 2D and $3 \mathrm{D}$, respectively. The area distribution of the triangles or the volume distribution of tetrahedrons can determine the properties of the underlying pattern.

The Voronoi and Delaunay tessellations can be applied on a 1D process which models traffic in time domain. In this case, the introduced distance-based measures are converted to time domain measures. The basic statistics of these measures for a PPP in one, two, and three dimensions, and their analogues in time domain, are summarized in Table 3.

In order to use the above mentioned measures as an analogue of iat, one needs to normalize their $\mathrm{CoV}$ with the $\mathrm{CoV}$ values of $i$ t in the time domain. For the complete homogeneity case, the $\mathrm{CoV}$ values are already zero, same as iat in the time domain. To normalize the $\mathrm{CoV}$ values of the complete random case to 1 , it is required to divide the measure by the values presented in Table 3. Figure 3 demonstrates realizations of processes with sub-Poisson, Poisson, and super-Poisson characteristics.

\subsubsection{Traffic Statistics}

Assuming that UEs have the same altitude, spatial traffic is modeled as a $2 \mathrm{D}$ point pattern $U \subset \mathbb{R}^{2}$ which is generated by a generator point process $\Phi_{U}$. In this thesis, the mean, $\mu_{m}$, and the $\mathrm{CoV}, C_{m}=\sigma_{m} / \mu_{m}$, are the desired statistics of traffic where $m$ is the traffic measure and $\sigma_{m}$ is the standard deviation of $m$ (the third-moment 
Table 3: Basic statistics of distance-based measures for a PPP in one, two and three dimensions and their analogues in time domain: $i$ is the process point index, $\lambda$ is the exponential distribution parameter for inter-arrival time and $\Lambda$ is the mean intensity of point processes.

\begin{tabular}{|c|c|c|c|c|c|}
\hline Measures & Time domain & Statistics & $1 \mathrm{D}$ & $2 \mathrm{D}$ & $3 \mathrm{D}$ \\
\hline Nearest neighbor & $\min \left\{I_{i}, I_{i+1}\right\}$ & $\begin{array}{l}\text { Mean } \\
\text { Variance } \\
\mathrm{CoV}\end{array}$ & $\begin{array}{l}0.5 \lambda^{-1} \\
0.25 \lambda^{-2} \\
1\end{array}$ & $\begin{array}{l}0.5 \Lambda^{-0.5} \\
0.0683 \Lambda^{-1} \\
0.653\end{array}$ & $\begin{array}{l}0.554 \Lambda^{-0.33} \\
0.04 \Lambda^{-0.66} \\
0.364\end{array}$ \\
\hline Voronoi volume & $\frac{I_{i}+I_{i+1}}{2}$ & $\begin{array}{l}\text { Mean } \\
\text { Variance } \\
\mathrm{CoV}\end{array}$ & $\begin{array}{l}\lambda^{-1} \\
\lambda^{-2} \\
1\end{array}$ & $\begin{array}{l}\Lambda^{-1} \\
0.28 \Lambda^{-2} \\
0.529\end{array}$ & $\begin{array}{l}\Lambda^{-1} \\
0.18 \Lambda^{-2} \\
0.424\end{array}$ \\
\hline Delaunay edge & $I_{i}$ & $\begin{array}{l}\text { Mean } \\
\text { Variance } \\
\mathrm{CoV}\end{array}$ & $\begin{array}{l}\lambda^{-1} \\
\lambda^{-2} \\
1\end{array}$ & $\begin{array}{l}1.131 \Lambda^{-0.5} \\
0.31 \Lambda^{-1} \\
0.492\end{array}$ & $\begin{array}{l}1.237 \Lambda^{-0.33} \\
0.185 \Lambda^{-0.66} \\
0.347\end{array}$ \\
\hline
\end{tabular}
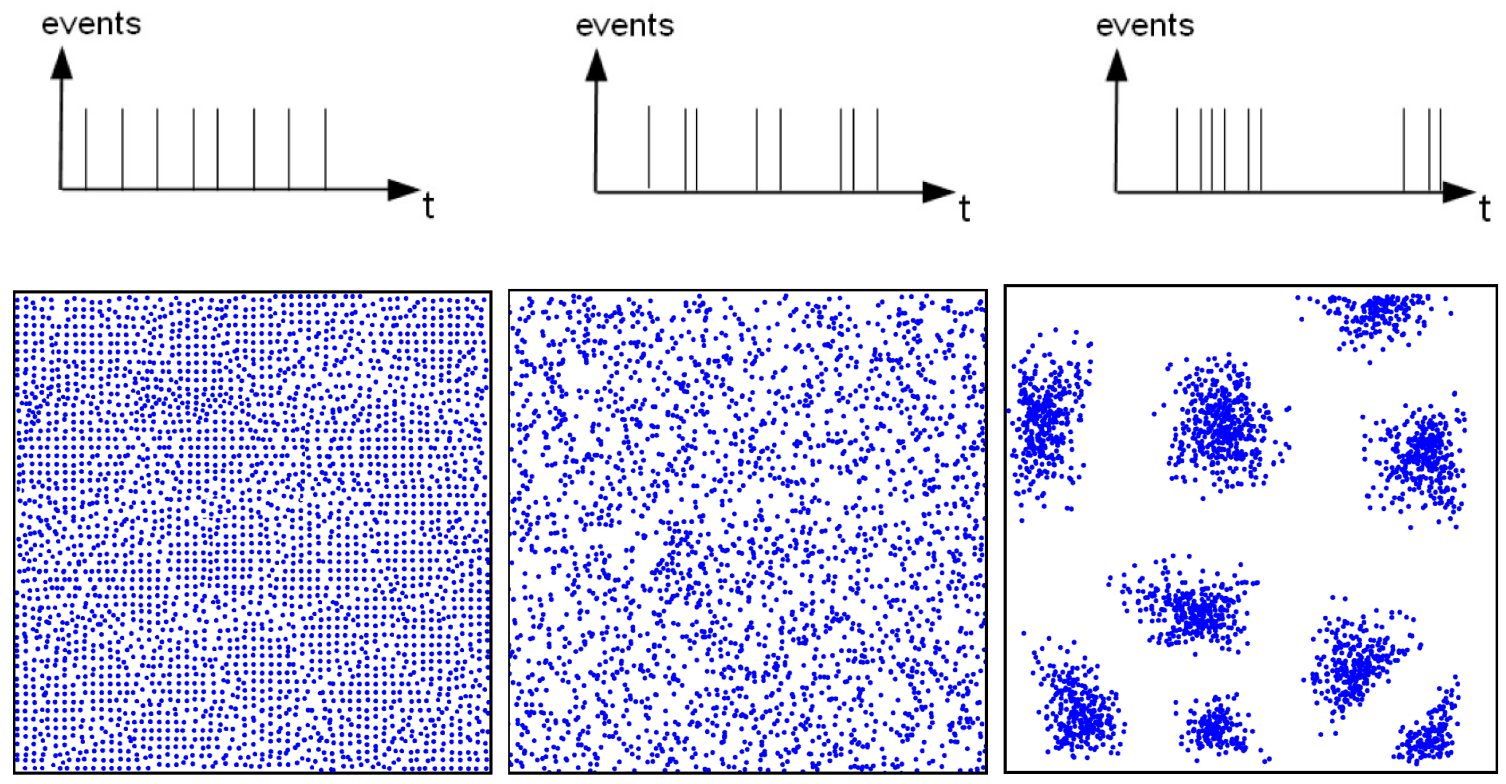

Figure 3: Domain similarity: Realizations of processes with sub-Poisson $(0<C<1)$, Poisson $(C=1)$, and super-Poisson $(C>1)$ characteristics, respectively, from left to right in time domain (top) and space domain (bottom). 
and auto-correlation are stated as future extensions in Section 2.6).

Along with the mean and the $\mathrm{CoV}$, which capture the heterogeneity of traffic, a very important statistic of traffic in space which affects the network performance is the bias of the UE distribution to the BS distribution (i.e., the correlation between the two distributions). BS locations can be modeled by a superposed 2D point pattern $B=\bigcup_{k=1}^{K} B_{k}, B_{k} \subset \mathbb{R}^{2}$, where $K$ is the number of tiers of BSs in heterogeneous infrastructures and $B_{k}$ is the set of BSs of type $k$ operating with power $P_{k}$ and generated by a point process $\Phi_{k}$ with density $\lambda_{k}$. The weighted-Voronoi tessellation of BSs divides the entire field into Voronoi cells associated with each BS consisting of the area closer, in terms of received signal power, to that BS than to any other BS. A sample realization is shown in Fig. 4(a).

To measure the joint distribution of UEs and BSs, we define the following potential function:

Definition 4. Potential Function: Every point $(x, y)$ in the field is associated with a potential value $P(x, y) \in[-1,+1]$. The $P$ function must have the following properties:

1. $P(x, y)=+1$, for cell center points,

2. $P(x, y)=-1$, for Voronoi cell edge points,

3. $\iint_{A_{i}} P(x, y) d x d y=0, \quad \forall i$, where $i$ is the index for $B S s$ and $A_{i}$ is the Voronoi cell area associated with $B S B_{i}$.

The first property assures that if a UE is at the cell center, its correlation with BSs is measured as +1 . The second property assures that if a UE is at the cell edge, its correlation with BSs is measured as -1 . The third property assures that if all UEs are distributed homogeneously (independent from BSs) in the area, their mean cross-correlation with BSs is equal to 0.

One may identify many functions which satisfy the requirements of the potential function defined above. In this thesis, we consider the simplest polynomial function.

Lemma 1. The simplest polynomial function which satisfies the requirements of the potential function is

$$
P(x, y)=\frac{-2(d(x, y))^{2}}{(D(x, y))^{2}}+1,
$$

where $d(x, y)$ is the distance of the point $(x, y)$ to the associated cell center, and $D(x, y)$ is the length of the line connecting the associated cell center to the associated Voronoi cell edge through point $(x, y)$ as shown in Fig. 5. 


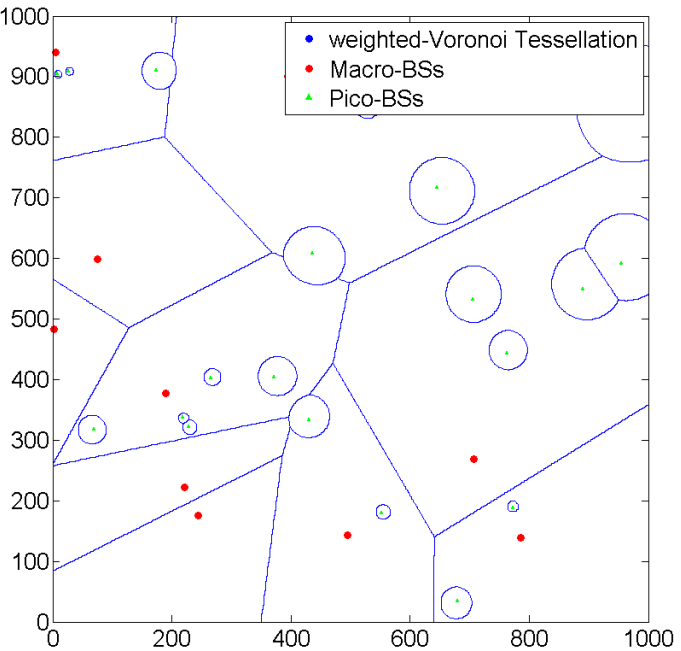

(a) BS weighted-Voronoi tessellation

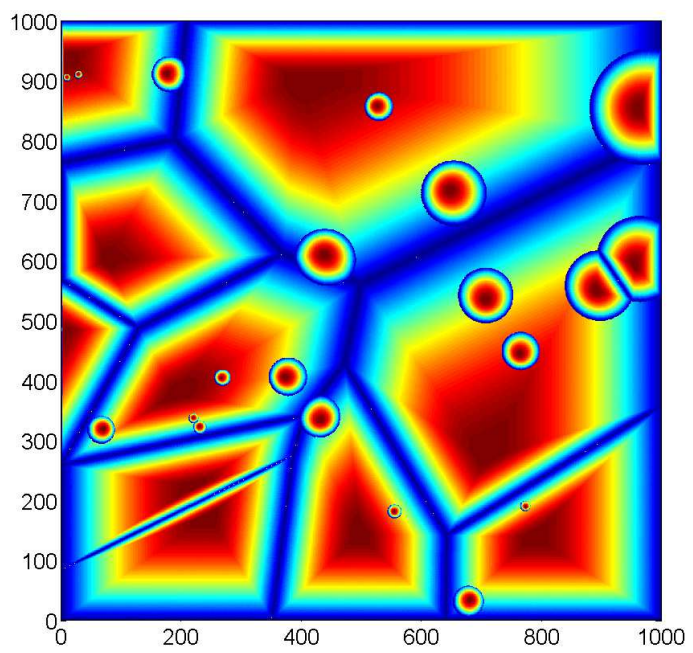

(b) potential distribution

Figure 4: The weighted-Voronoi tessellation of BSs divides the whole field into cells associated with each BS consisting of all points closer, in terms of received signal power, to that BS than to any other BS. Every point in the field is then associated with a potential value between -1 and +1 , according to (3).

\section{Proof. See Appendix.}

Figure 4(b) illustrates the potential distribution associated with the Voronoi tessellation in Fig. 4(a).

Using this potential function, the joint moments

$$
\mathbb{E}\left[P^{i} \Lambda^{j}\right], i, j \geqslant 0
$$

and joint central moments

$$
\mathbb{E}\left[\left(P-\mu_{P}\right)^{i}\left(\Lambda-\mu_{\Lambda}\right)^{j}\right], i, j \geqslant 0
$$

can be calculated, where $\mu_{P}$ is the mean value of $P, \mu_{\Lambda}$ is the mean value of UE density $\Lambda$, and $\mathbb{E}[x]$ is the expected value of $x[70]$. To have a normalized measure, we use correlation coefficient which is defined as

$$
\rho=\frac{\sigma_{P \Lambda}}{\sigma_{P} \sigma_{\Lambda}}
$$




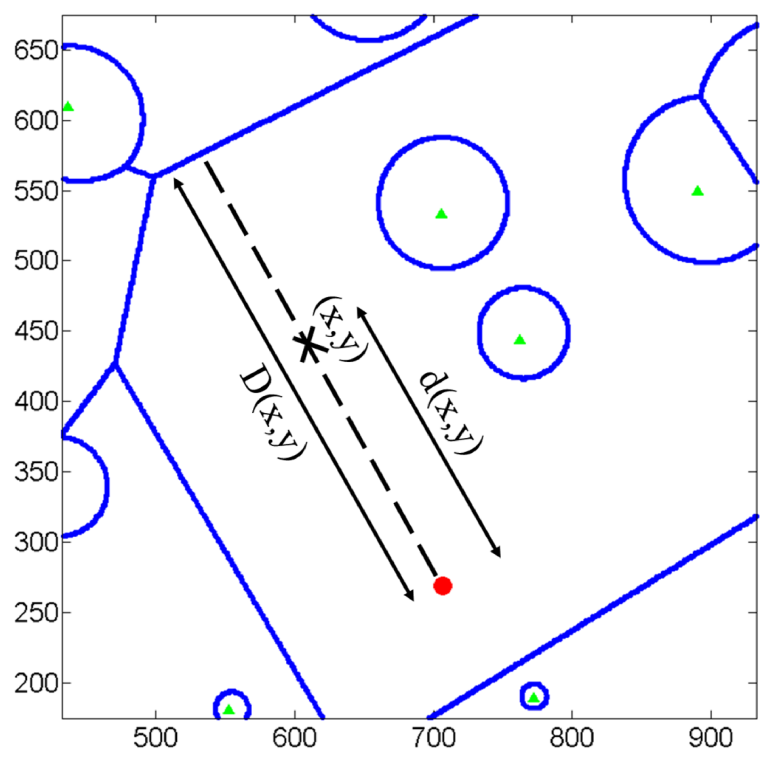

Figure 5: The $d(x, y)$ is the distance of the point $(x, y)$ to the associated cell center, and $D(x, y)$ is the length of the line connecting the associated cell center to the associated Voronoi cell edge through point $(x, y)$.

where

$$
\sigma_{P \Lambda}=\mathbb{E}\left[\left(P-\mu_{P}\right)\left(\Lambda-\mu_{\Lambda}\right)\right]
$$

is the covariance of $P$ and $\Lambda, \sigma_{P}$ is the standard deviation of $P$, and $\sigma_{\Lambda}$ is the standard deviation of $\Lambda$. Therefore, $\rho$ can be defined as

$$
\rho=\frac{\iint\left(\Lambda(x, y)-\mu_{\Lambda}\right)\left(P(x, y)-\mu_{P}\right) d x d y}{\sqrt{\left(\iint\left(\Lambda(x, y)-\mu_{\Lambda}\right)^{2} d x d y\right)\left(\iint\left(P(x, y)-\mu_{P}\right)^{2} d x d y\right)}} .
$$

For a UE pattern $U$ (a realization of $\Phi_{U}$ ), the correlation coefficient is calculated as

$$
\rho=\frac{\sum_{u \in U} P_{u}}{|U|}
$$

where $P_{u}$ is the potential value at point $u$ and $|U|$ is the number of points in $U$. A pattern with $\rho=+1$ means that all UEs have gathered at the cell centers, a pattern with $\rho=0$ means that the UE distribution is independent from the BS distribution, and a pattern with $\rho=-1$ means that all UEs have gathered at the cell edges. 


\subsection{Heterogeneous Traffic Generation}

A basic traffic generation method is presented in Section 2.4.1, which is further improved in Section 2.4.2.

\subsubsection{The Basic Method}

UEs in a heterogeneous wireless cellular network are attracted to social attractors (SA) such as buildings, bus stations, shopping centers, and other social places. Furthermore, with the proliferation of small-cells and the deployment of BSs near potential $\mathrm{SAs}$, the distance between SAs and BSs is decreasing. The requirement is a traffic generation method which is able to generate most of the possible traffic distributions including the following:

- situations in which UEs are not attracted to SAs at all and are completely random, while SAs in turn are distributed independently from BSs,

- situations in which UEs are not attracted to SAs at all and are completely random, while SAs are close to BSs,

- situations in which UEs are highly attracted to SAs, while SAs are distributed independently from BSs,

- situations in which UEs are highly attracted to SAs, and SAs are close to BSs.

Covering the entire range of the above discussed cases, our proposed traffic generation method is described as follows. Obviously, the proposed method is just one way of generating adjustable heterogeneous and BS-correlated traffic; i.e., it is not the only way. More accurate methods can be developed based on the real world data analysis.

Starting with a Poissonian distribution of BSs $(B)$, including macro-BSs, pico-BSs, and femto-BSs, an independent Poissonian distribution of SAs $(S)$, and an independent distribution of UEs $(U)$, we move every SA $\left(\vec{S}_{i}\right)$ towards its closest BS, in terms of the received signal power $\left(\vec{B}_{S_{i}}\right)$, by a factor of $\alpha \in[0,1]$; so the SA's new location $\left(\vec{S}_{i}^{n e w}\right)$ is calculated as

$$
\vec{S}_{i}^{\text {new }}=\alpha \vec{B}_{S_{i}}+(1-\alpha) \vec{S}_{i}
$$

Then we move every UE $\left(\vec{U}_{i}\right)$ towards its closest SA, in terms of the Euclidean distance $\left(\vec{S}_{U_{i}}\right)$, by a factor of $\beta \in[01]$; so the UE's new location $\left(\vec{U}_{i}^{\text {new }}\right)$ is calculated 
as

$$
\vec{U}_{i}^{n e w}=\beta \vec{S}_{U_{i}}+(1-\beta) \vec{U}_{i}
$$

The initial distribution of UEs can be Poissonian $(C=1)$ or deterministic $(C=0)$. In this chapter, to generate sub-Poisson traffic $(C<1)$, we start with a deterministic lattice.

The proposed method is general enough to generate traffic with negative bias to BSs, i.e., UEs gathering at cell edges. To generate this type of traffic, UEs must be moved to cell edges instead of cell centers.

\subsubsection{The Enhanced Method}

An undesired property of the basic method described in 2.4.1 is that when UEs move towards the SAs, some areas of the network become empty having no UEs. In other words, the UE distribution shape stays unchanged and it only shrinks to a smaller area. Figure 20(a) shows the CDF of the UE density of network after moving UEs towards SAs with different $\beta$ values using the basic method. Even for small values of $\beta$ (e.g., $\beta=0.4$ ), the SA Voronoi cell edge area has no UEs.

To resolve this undesired feature of the basic method, we introduce an enhanced method as follows. Instead of having a fixed value for $\beta$, in the enhanced method we model $\beta$ as a random variable with

$$
\beta \sim \mathrm{N}\left(\mu_{\beta}, \sigma_{\beta}\right)
$$

where the mean $\mu_{\beta} \in[0,1]$ indicates the closeness factor of UEs to SAs (an accurate model based on the real world measurements may adopt another distribution for $\beta$, however, since we don't have access to real traffic measurements, in this thesis we choose normal distribution). In (12), the $\sigma_{\beta}$ value should have the following characteristics:

- $\beta \rightarrow 0 \Rightarrow \sigma_{\beta} \rightarrow 0$

- $\beta \rightarrow 1 \Rightarrow \sigma_{\beta} \rightarrow 0$

- $\beta \rightarrow 0.5 \Rightarrow \sigma_{\beta}$ is maximized.

The $\sigma_{\beta}$ value should also be selected in such a way that the probability of $\beta$ falling 


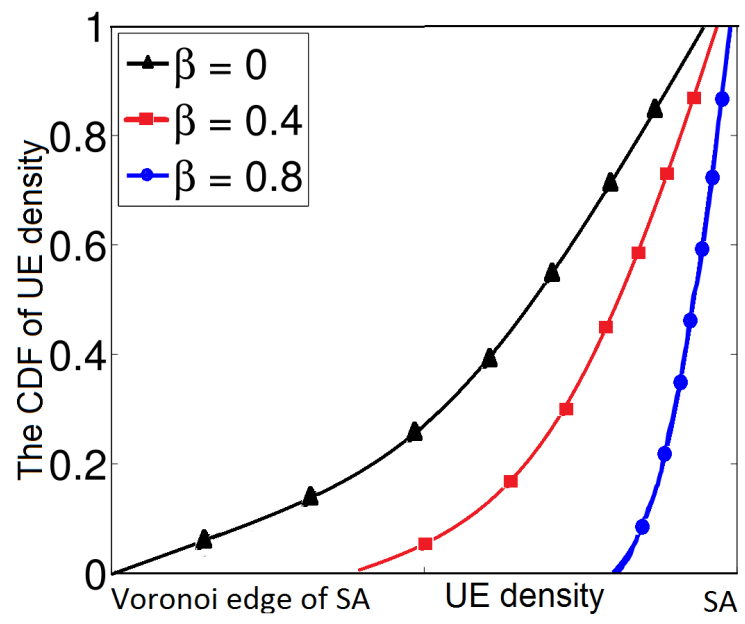

(a) $\beta$ is fixed and deterministic

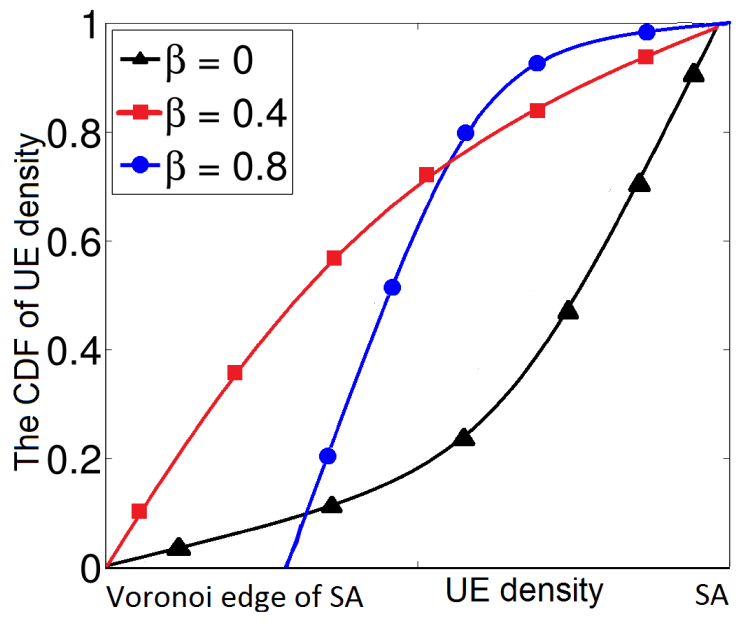

(b) $\beta$ is random (normal distribution)

Figure 6: The CDF of the distribution of UEs in the Voronoi cell area of SAs: (a) When $\beta$ is deterministic, the UEs are moved towards SAs but the UE distribution shape is fixed and the Voronoi cell edge area of SAs remains empty with no UEs. (b) When $\beta$ is a random variable with normal distribution, even for high $\beta$ values, the Voronoi cell edges are not empty and there is a low probability for UEs existing at cell edges.

outside $[0,1]$ should be negligible. In this thesis, we set $\sigma_{\beta}$ to be

$$
\sigma_{\beta}=\frac{0.5-\left|\mu_{\beta}-0.5\right|}{3}
$$

thus the probability of $\beta$ falling outside $[0,1]$ will be $0.1 \%$ as shown in Fig. 7 .

Figure 20(b) shows the CDF of the UE density of network after moving UEs towards SAs with different $\beta$ values using the enhanced method. 


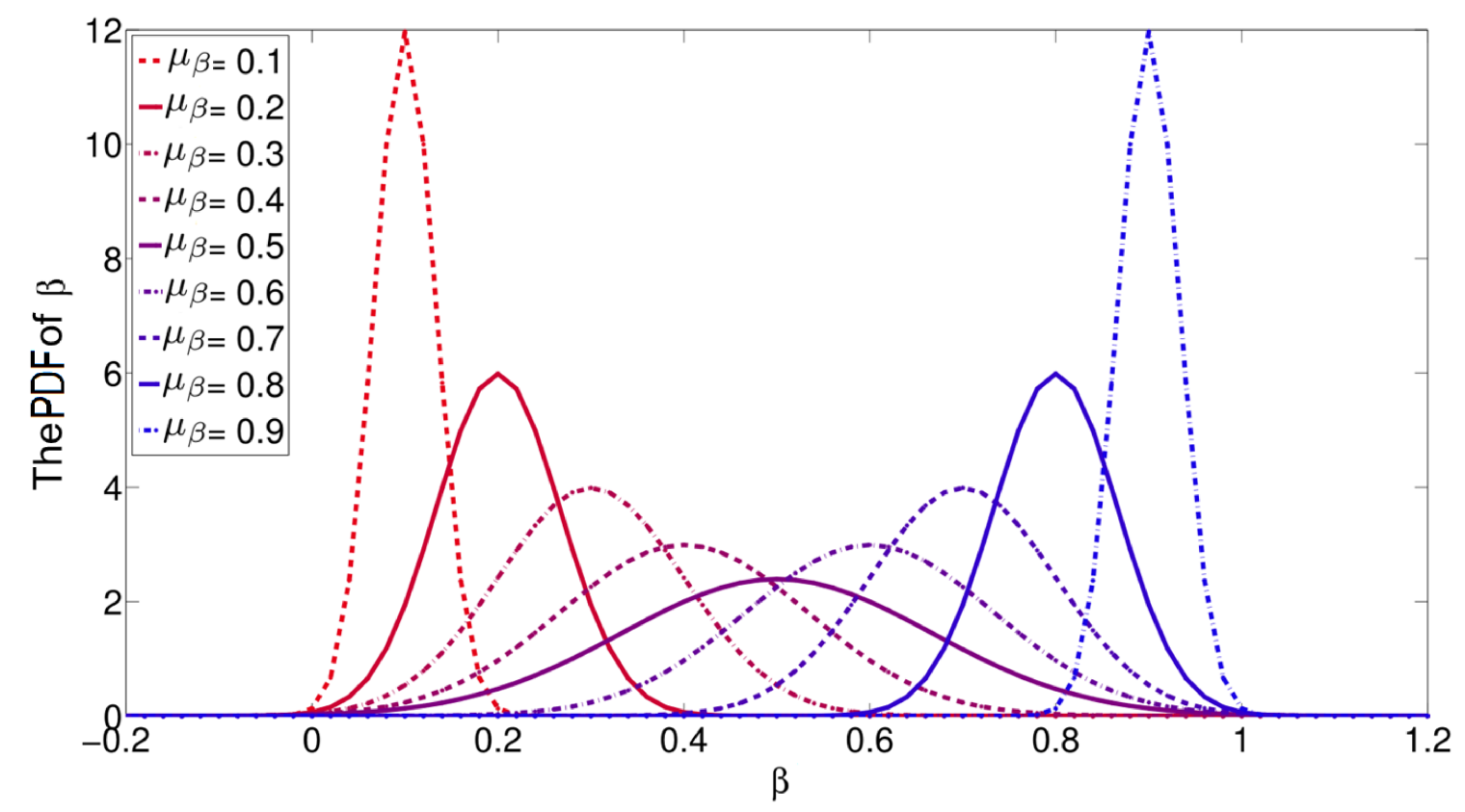

Figure 7: $\beta$ is distributed with normal PDF with a mean value of $\mu_{\beta}$.

Figure 8 illustrates various scenarios with different characteristics which can be generated by the proposed traffic generation method. 


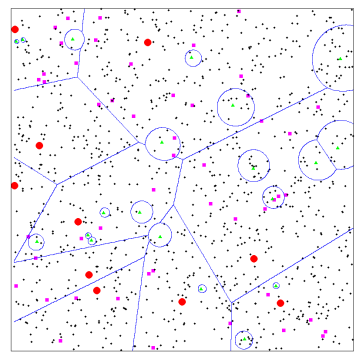

(a) $\alpha=0$ and $\beta=0$

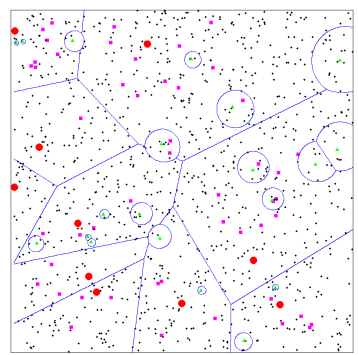

(e) $\alpha=0.3$ and $\beta=0$

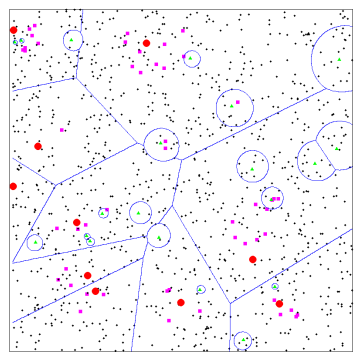

(i) $\alpha=0.6$ and $\beta=0$

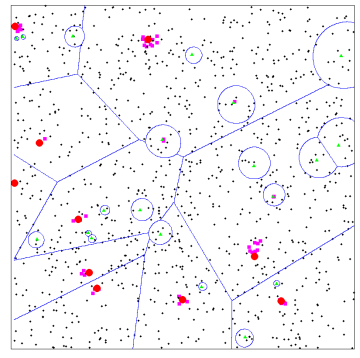

(m) $\alpha=0.9$ and $\beta=0$

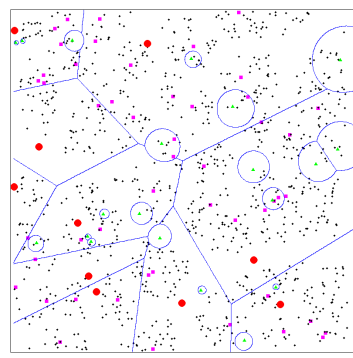

(b) $\alpha=0$ and $\beta=0.3$

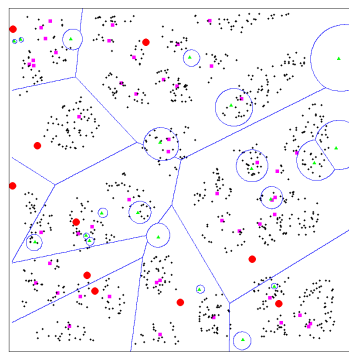

(f) $\alpha=0.3$ and $\beta=0.3$

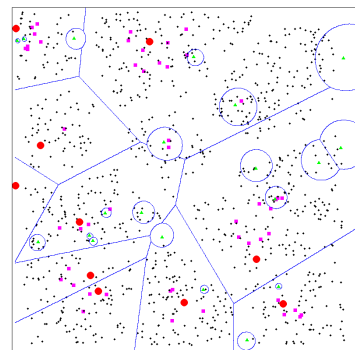

(j) $\alpha=0.6$ and $\beta=0.3$

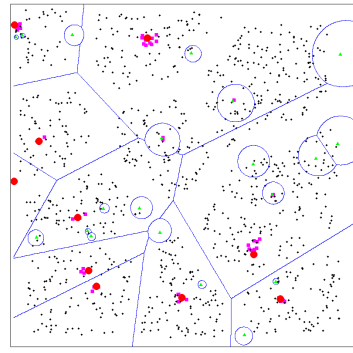

(n) $\alpha=0.9$ and $\beta=0.3$

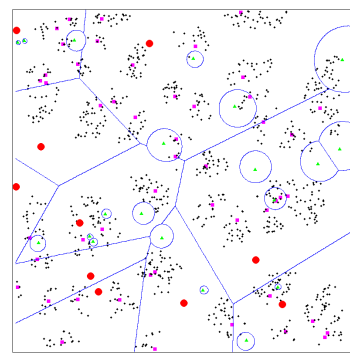

(c) $\alpha=0$ and $\beta=0.6$

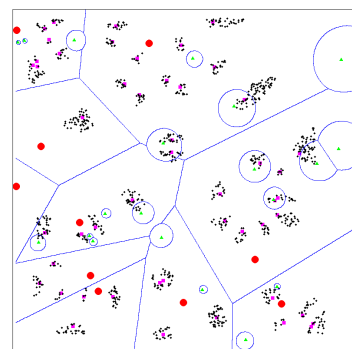

(g) $\alpha=0.3$ and $\beta=0.6$

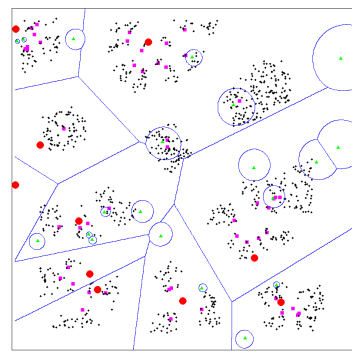

(k) $\alpha=0.6$ and $\beta=0.6$

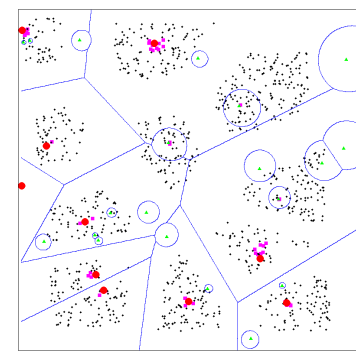

(o) $\alpha=0.9$ and $\beta=0.6$

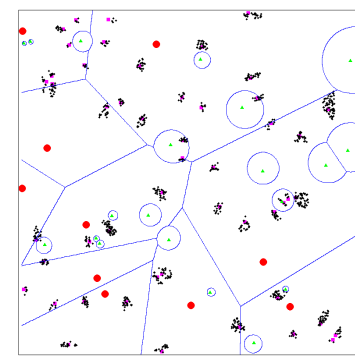

(d) $\alpha=0$ and $\beta=0.9$

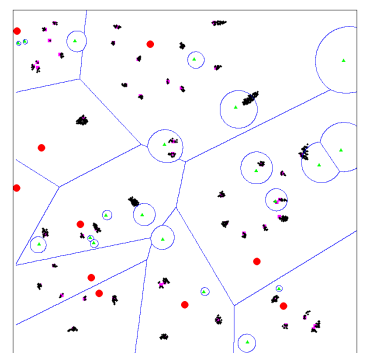

(h) $\alpha=0.3$ and $\beta=0.9$

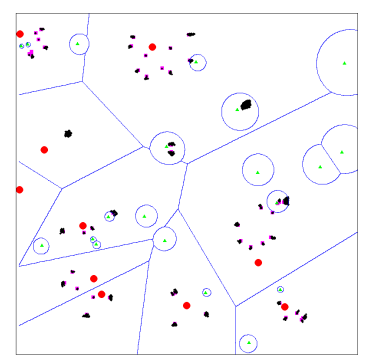

(l) $\alpha=0.6$ and $\beta=0.9$

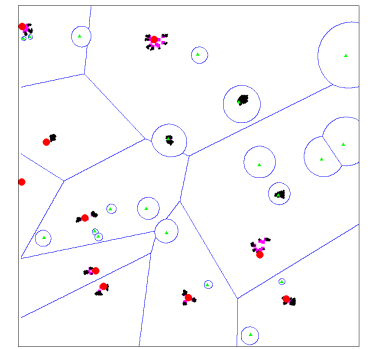

(p) $\alpha=0.9$ and $\beta=0.9$

Figure 8: Traffic distribution scenarios: By regulating only two parameters, $\alpha$ (the closeness indicator of social attractors to $\mathrm{BSs}$ ), and $\beta$ (the closeness indicator of UEs to social attractors) from 0 to 1 , traffic distributions with various properties $(\mathrm{CoV}$ and BS-correlation) can be generated. The big circles denote macro-BSs, the triangles denote pico-BSs, the lines are the edges of the weighted-Voronoi tessellation of BSs, squares denote social attractors and small circles represent UEs. 
In our modeling methodology, the TGIPs are $\alpha$ and $\beta$ which are the internal parameters of the traffic generator, and the traffic statistics of interest are $C$ and $\rho$. To make the mapping (i.e., the look-up table generation) from the statistics ( $C$ and $\rho$ in our method) to the TGIPs ( $\alpha$ and $\beta$ in our method), we first generate traffic patterns with different values of TGIPs and then measure the associated statistics. Using a fitting procedure, the resulting mapping can be expressed as

$$
C=F_{1}(\alpha, \beta)
$$

and

$$
\rho=F_{2}(\alpha, \beta)
$$

$F_{1}$ and $F_{2}$ are monotonically non-decreasing functions due to the fact that the $\mathrm{CoV}$ and correlation coefficient values are both non-decreasing with increasing $\alpha$ and $\beta$. Therefore, the final maps from the desired statistics to TGIPs can be obtained by the corresponding inverse operations as follows:

$$
\alpha=H_{1}(C, \rho)
$$

and

$$
\beta=H_{2}(C, \rho)
$$

\subsection{Numerical Results}

Following the methodology described in Section 2.2, the traffic measurement method discussed in Section 2.3, and the traffic generation model described in Section 2.4, the simulation results are presented in this section. The traffic modeling results are presented in Section 2.5.1, and the heterogeneous wireless cellular network performance analysis results are presented in Section 2.5.2.

\subsubsection{Traffic Modeling Results}

Considering a two-tier superposition of BSs (i.e., macro-BSs and pico-BSs), 10 macroBSs and 20 pico-BSs are distributed in a $1000 \mathrm{~m} \times 1000 \mathrm{~m}$ square field. The distribution of BSs is assumed to be PPP. 50 SAs are distributed by an independent PPP. UEs are distributed using the traffic generation method described in Section 2.4 with 
parameters $\alpha$ and $\beta$. The simulation is repeated for 1000 random drops.

The first step in traffic measurement is to select the appropriate traffic measure. To compare the proposed spatial traffic measures with the existing distance-based nearest-neighbor distance measure, with $\alpha$ fixed at 0 , we increased $\beta$ from 0 to 1 and calculated different traffic measures for each $\beta$ value. Figure 9 illustrates the $\mathrm{CoV}$ values of various distance-based traffic measures introduced in this thesis.

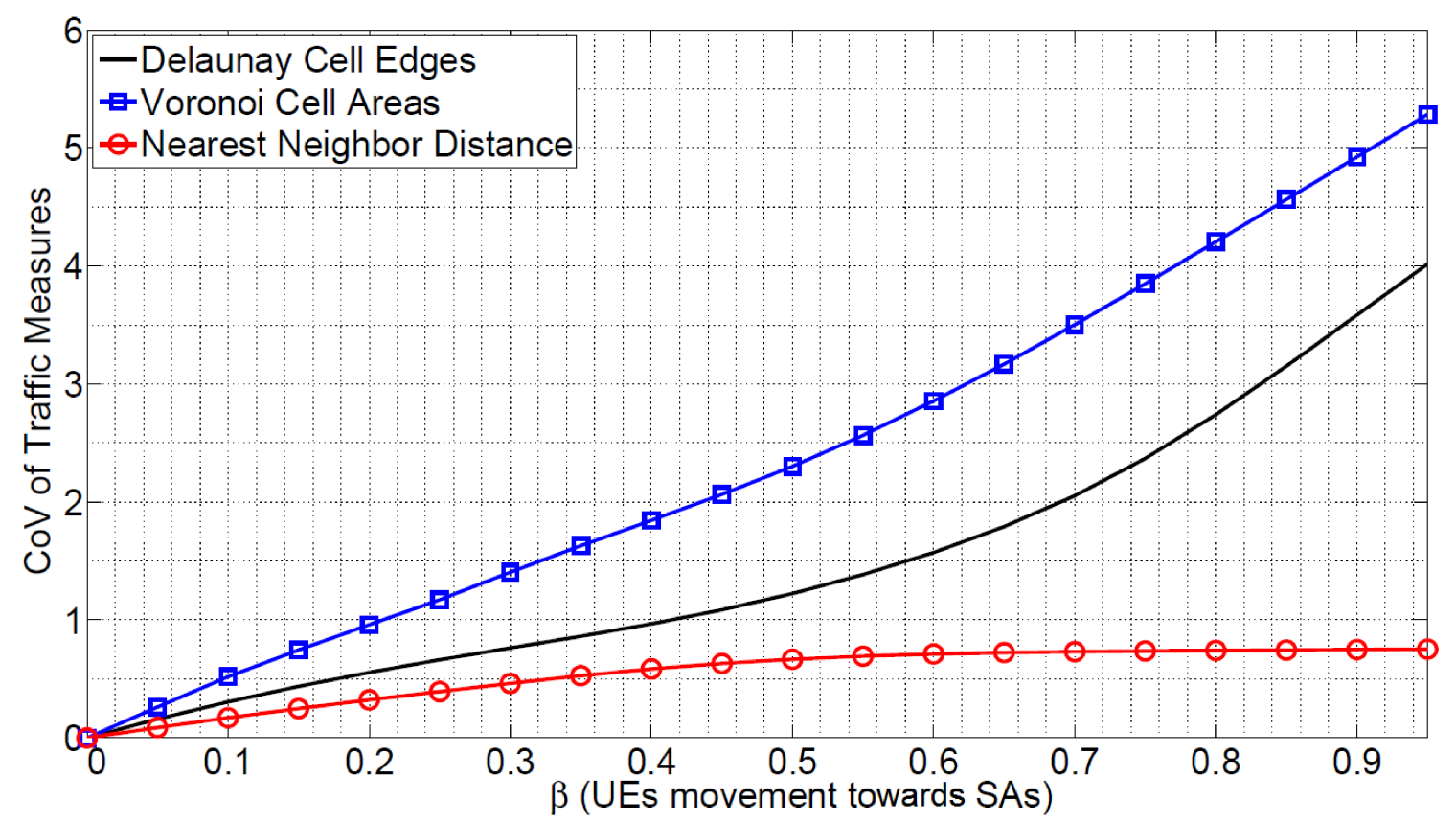

Figure 9: To compare the proposed distance-based traffic measures with the existing nearest-neighbor distance measure, we fixed the $\alpha$ value to zero $(\alpha=0)$ and changed the $\beta$ value from 0 to 1 and calculated the $\mathrm{CoV}$ of different measures. Nearest-neighbor distance cannot capture the traffic heterogeneity and stays constant in super-Poisson region.

The CoVs of all the measures are normalized to 1 at the Poisson case by dividing the CoVs by their expected values (presented in Table 3). Figure 10 shows the normalized results. 


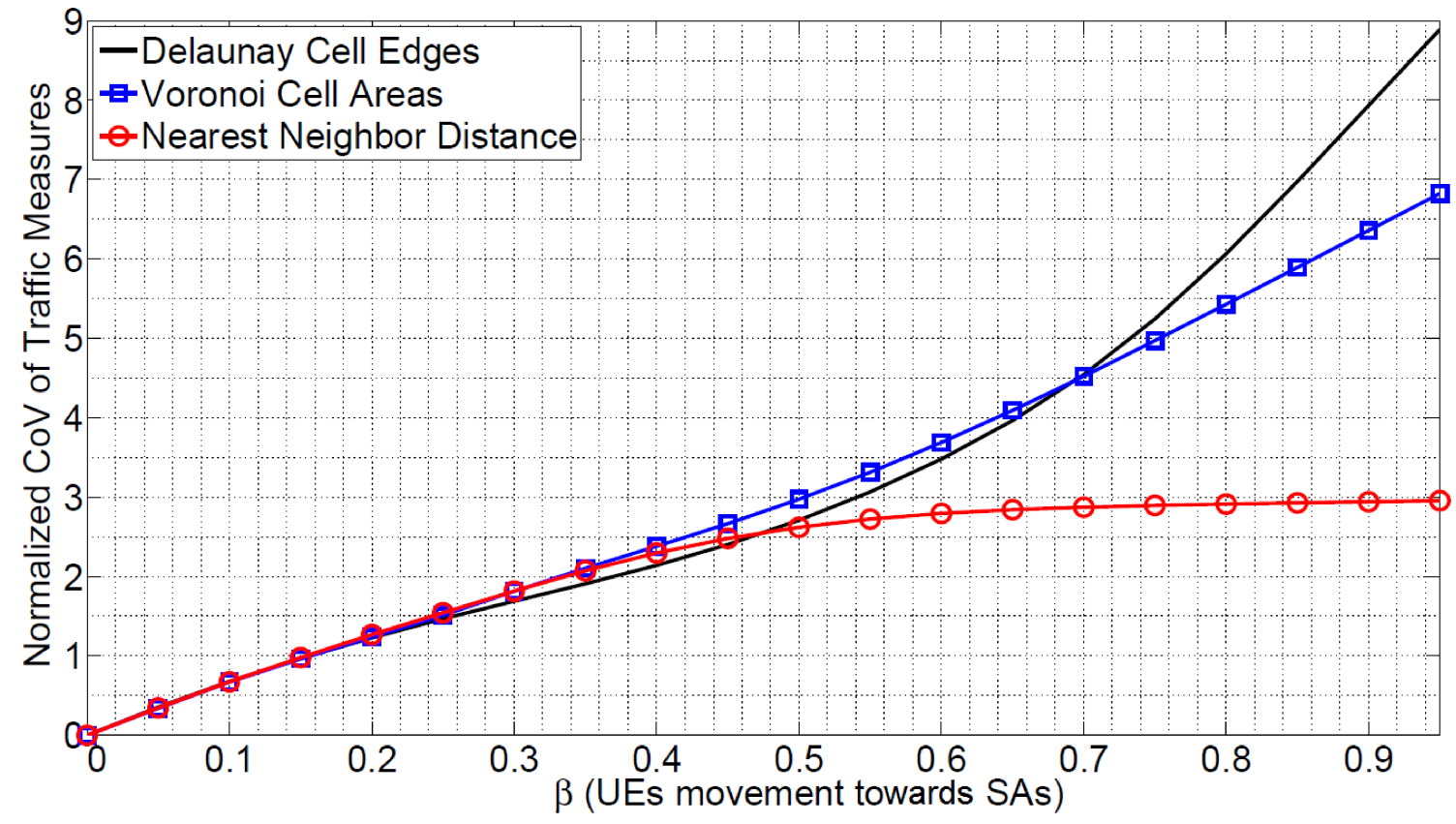

Figure 10: To be consistent with time domain traffic measurement, we divide the $\mathrm{CoV}$ of the space traffic measures by the convergence value at Poisson patterns. The normalized $\mathrm{CoV}$ values are used in this thesis.

As it can be seen in Fig. 10, the nearest-neighbor distance measure cannot capture the traffic heterogeneity as it stays constant in the super-Poisson region. On the other hand, the two proposed measures capture the traffic heterogeneity for all $\mathrm{CoV}$ values. The Voronoi cell area is preferable to the Delaunay cell edge length for two reasons: First, there exists a Voronoi cell area associated with each user, while a Delaunay cell edge length cannot be associated with one particular UE. Secondly, the slope of the Voronoi cell area with respect to $\beta$ is higher than the slope of the Delaunay cell edge length with respect to $\beta$; thus the Voronoi cell area can capture the traffic heterogeneity with a higher resolution. In the remainder of this chapter we use the Voronoi cell area as the traffic measure.

The next step in traffic modeling is to generate a map from TGIPs to traffic statistics. Figures 11 and 12 demonstrate the calculated $\mathrm{CoV}$ and correlation coefficient values, respectively, for different values of $\alpha$ and $\beta$, both ranging from 0 to 1 . 


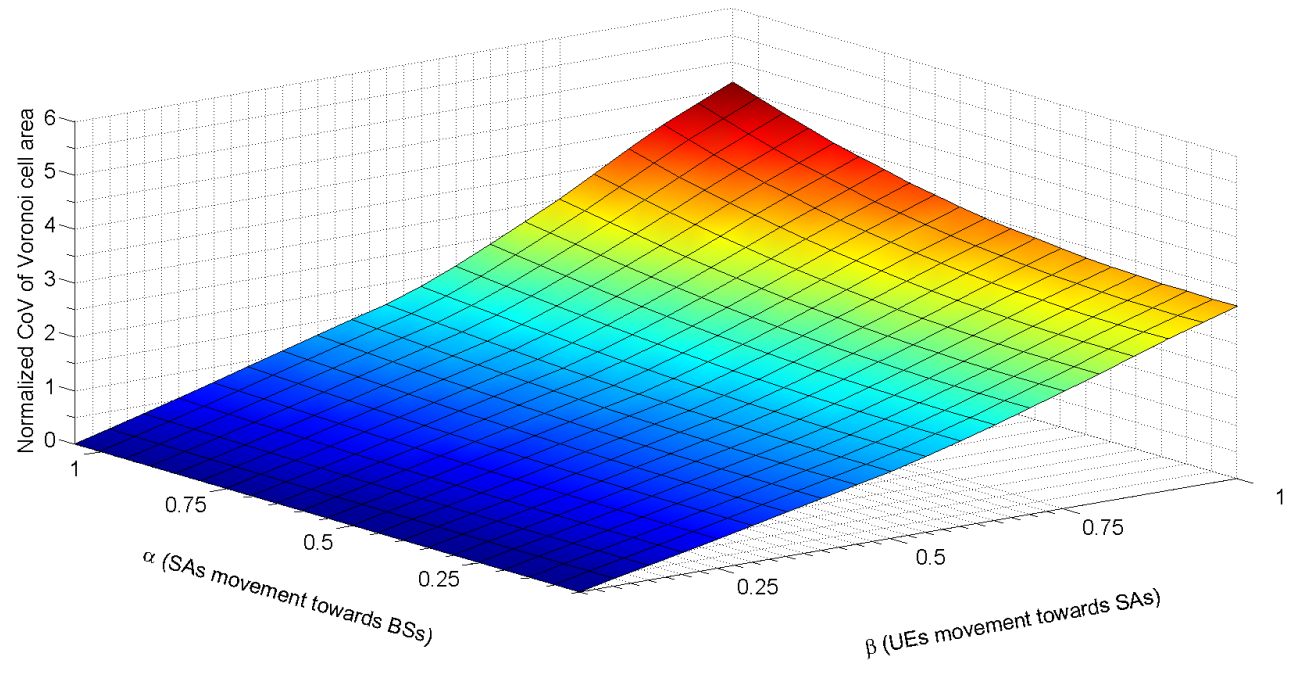

Figure 11: The normalized CoV of Voronoi cell area is calculated for traffic generated with different values of $\alpha$ and $\beta$.

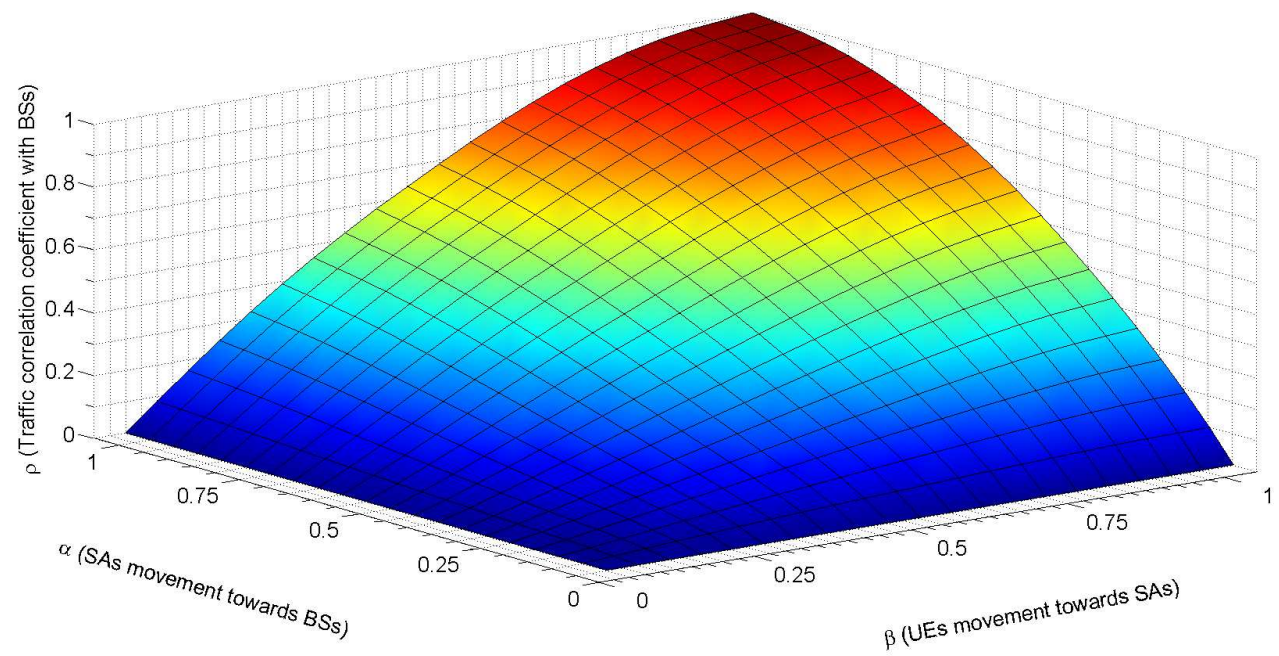

Figure 12: The correlation coefficient (refer to (9)) between UEs and BSs is calculated for traffic generated with different values of $\alpha$ and $\beta$.

An important observation from the maps in Figures 11 and 12 is that the $\mathrm{CoV}$ and BS-correlation are not independent. In other words, the range of achievable $\mathrm{CoV}$ 
values for each correlation coefficient value is different. This is due to the fact that when UEs are biased towards BSs, UEs are attracted towards the points of interest, and this automatically shapes clusters; which results in a degree of clustering and increases the $\mathrm{CoV}$.

To make a comparison with the existing models in the literature, we should note that the prevailing spatial model used for UE distribution in the literature is the uniform PPP. To compare the PPP model to our model we should say that the PPP corresponds to just one specific point in the whole space of the possible spatial distribution situations $(\mathrm{CoV}$ (heterogeneity) $=1$ and $\rho$ (BS-correlation $)=0)$. There are few models in the literature which consider heterogeneous or BS-correlated traffic modeling. To the best of the authors' knowledge the most appropriate of those is the one presented in [22] which is a BS-correlated model. However, the model in [22] is capable of generating a limited sub-space of the whole traffic possibilities. Figure 13 illustrates the feasible region of $\mathrm{CoV}$ and correlation coefficient values generated by our model versus the model presented in [22].

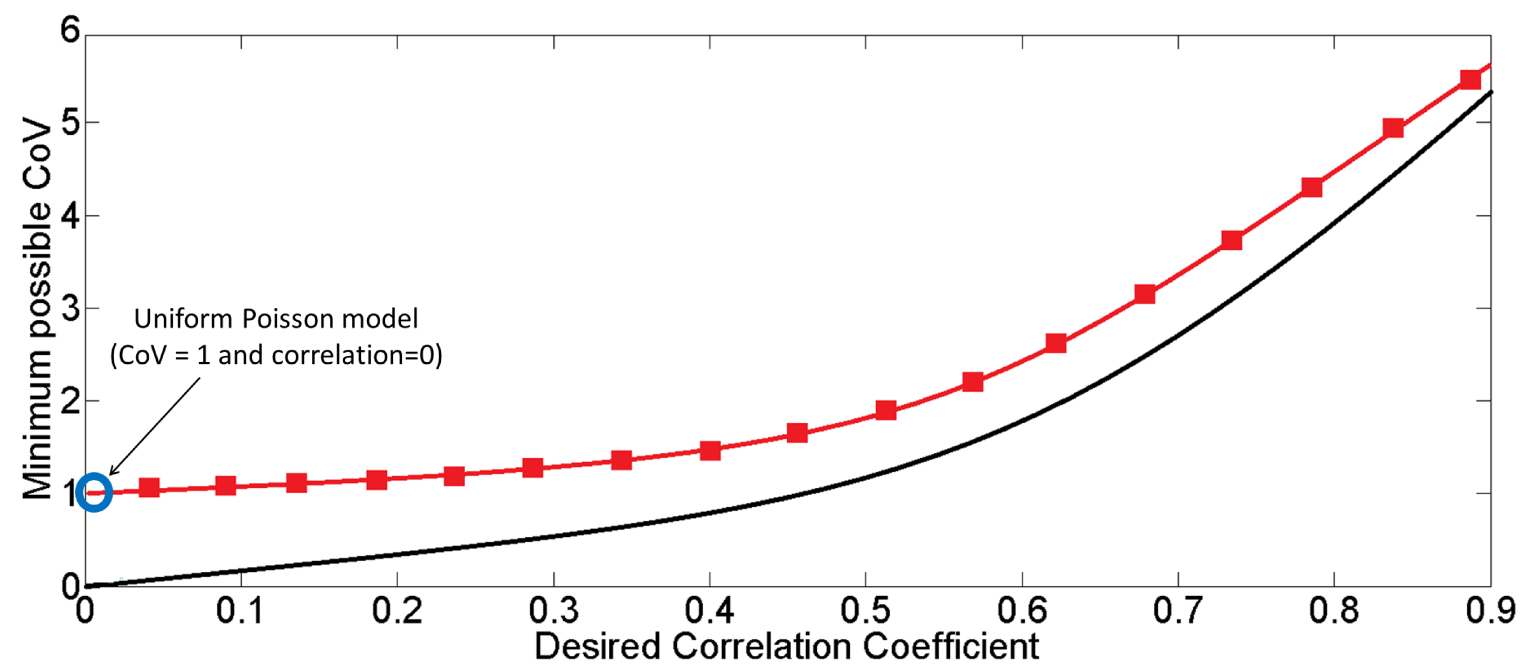

Figure 13: The feasible normalized CoV values for different values of correlation coefficient are shown. Traffic with high correlation with BSs cannot have low normalized $\mathrm{CoV}$ values because high correlation means that UEs are gathered at cell centers. All the region above the solid line is the possible traffic generation using our proposed method.

The next step in traffic modeling is to translate the desired statistics to appropriate 
TGIPs which can generate traffic with the desired statistics. Towards that end, the statistical properties of the generated traffic are measured. Since the process of traffic generation is a random process (e.g., $\beta$ is a random variable), the measured statistics are not constant and have a deviation around their expected values. The deviation of the measured statistics from the desired statistics determines the accuracy of the method. Figures 14 and 15 illustrate the measured statistics versus the desired statistics.

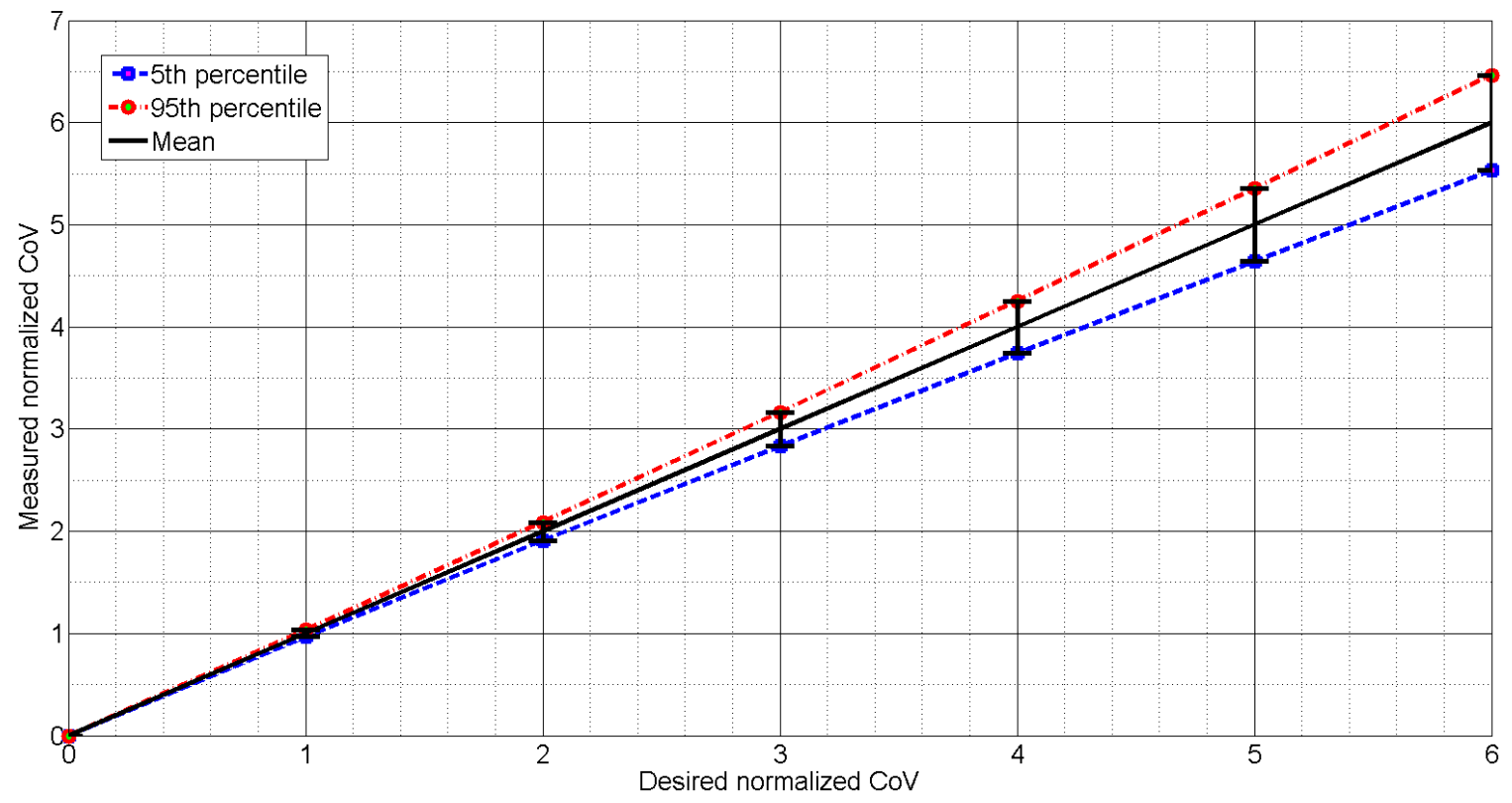

Figure 14: The horizontal axis shows the desired normalized CoV. The desired normalized $\mathrm{CoV}$ is mapped to the associated $\alpha$ and $\beta$ values which are used to generate traffic. The vertical axis shows the calculated normalized $\mathrm{CoV}$ from the generated traffic patterns (for each desired $C$ value, the traffic is generated for all feasible $\rho$ values and the $\mathrm{CoV}$ is averaged out). 


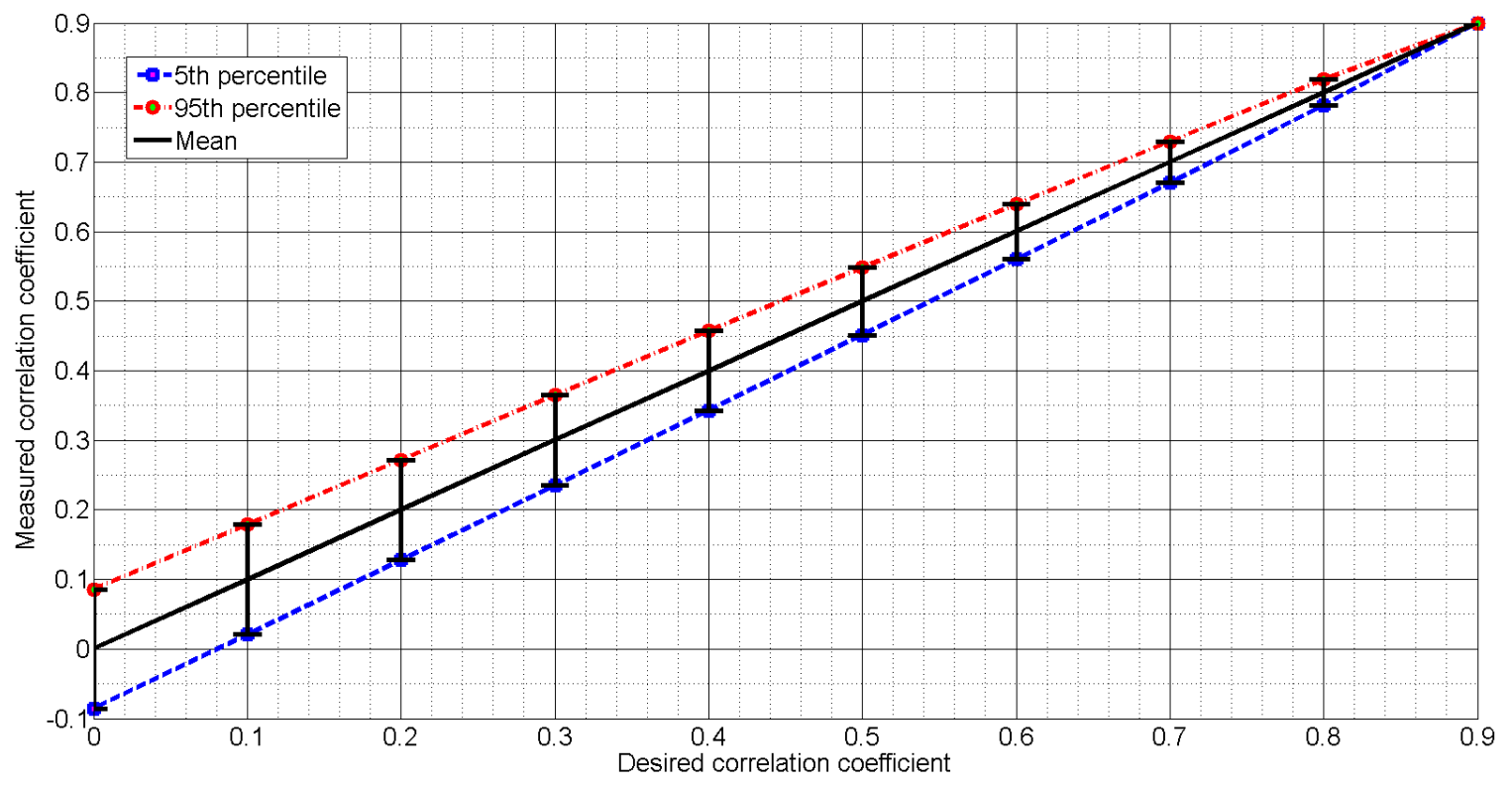

Figure 15: The horizontal axis shows the desired $\rho$. The desired $\rho$ is mapped to the associated $\alpha$ and $\beta$ values which are used to generate traffic. The vertical axis shows the calculated $\rho$ from the generated traffic patterns (for each desired $\rho$ value, the traffic is generated for all feasible normalized $\mathrm{CoV}$ values and the correlation coefficient is averaged out).

\subsubsection{Network Performance Analysis Results}

The performance of a downlink LTE cellular network is analyzed in this section. In a field of $1000 \mathrm{~m} \times 1000 \mathrm{~m}$, UEs are distributed using the traffic generation method described in Section 2.4. The simulation parameters are summarized in Table 5 $[71,72]$. 
Table 4: Simulation parameters for Chapter 2.

\begin{tabular}{|c|c|}
\hline PARAMETER & VALUE \\
\hline Mean number of UEs & 1000 \\
\hline Number of macro-BSs & 10 \\
\hline Number of pico-BSs & 20 \\
\hline Number of SAs & 50 \\
\hline BSs distribution & PPP \\
\hline BS antenna height & $10 \mathrm{~m}$ \\
\hline Number of drops & 1000 \\
\hline Bandwidth (downlink) & $20 \mathrm{MHz}$ \\
\hline Noise power per RB & $-174 \mathrm{dBm} / \mathrm{Hz}$ \\
\hline Carrier frequency & $2.5 \mathrm{GHz}$ \\
\hline Total macro-BS transmit power & $37 \mathrm{dBm}$ \\
\hline Total pico-BS transmit power & $17 \mathrm{dBm}$ \\
\hline Path loss and shaddowing & Based on UMi scenario [3] \\
\hline BS antenna gain (boresight) & $17 \mathrm{dBi}$ \\
\hline UE antenna gain & $0 \mathrm{dBi}$ \\
\hline Time domain traffic model & Full buffer \\
\hline Antenna model & Omni-directional \\
\hline BS down tilt & 12 degrees \\
\hline UE antenna height & $1.5 \mathrm{~m}$ \\
\hline Shadowing model & log-normal with std: LoS: 3, NLoS: 6 \\
\hline Fading model & no fading \\
\hline Scheduling & Proportional fair [73] \\
\hline
\end{tabular}


The mean value of the rates of all the network users, and the coverage probability with a minimum SINR threshold of $10 \mathrm{~dB}$ versus the feasible traffic statistics (for 1000 drops) are presented in Fig. 16 and Fig. 17, respectively.

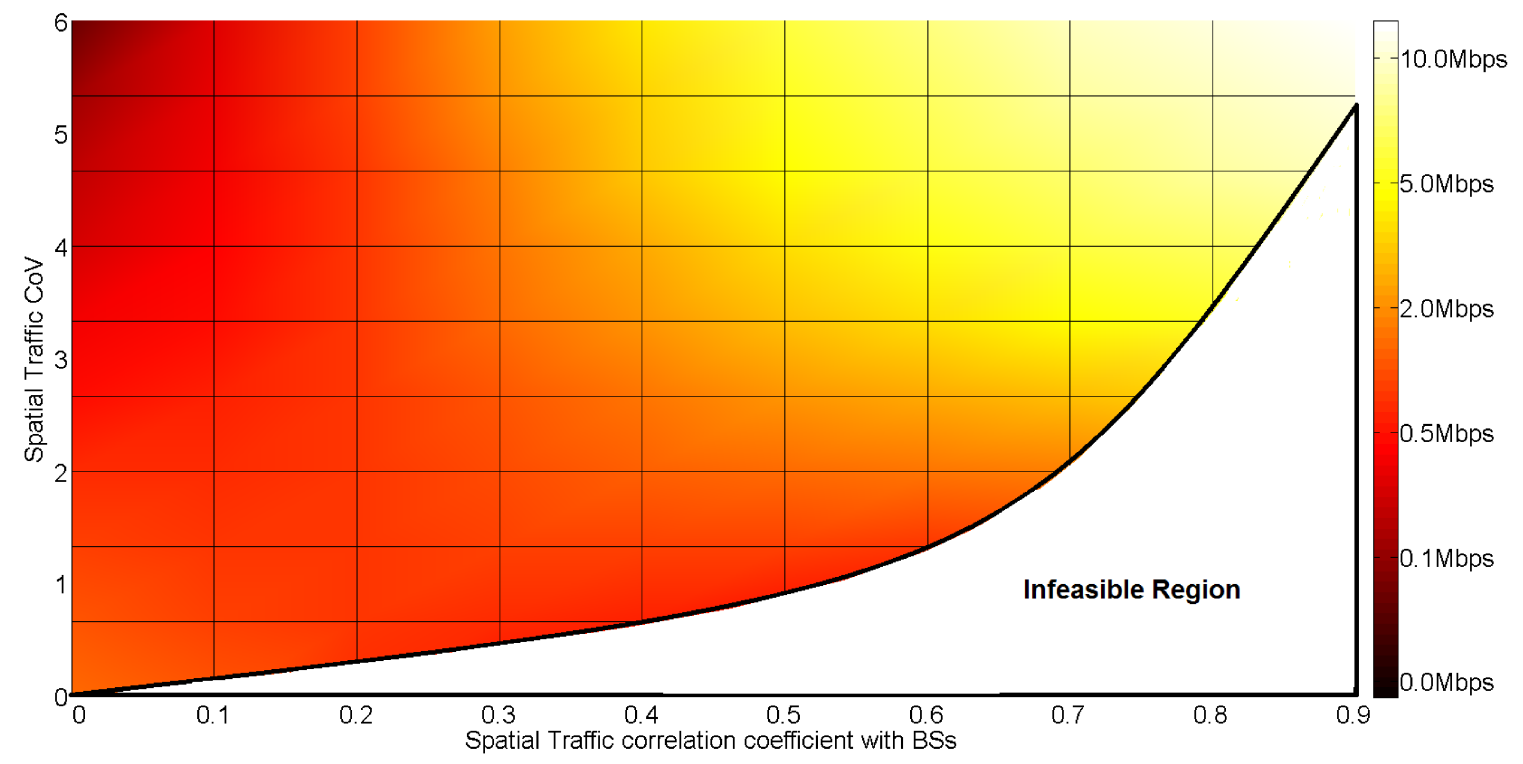

Figure 16: The mean user rates is shown for the feasible normalized CoV and correlation coefficient region. 


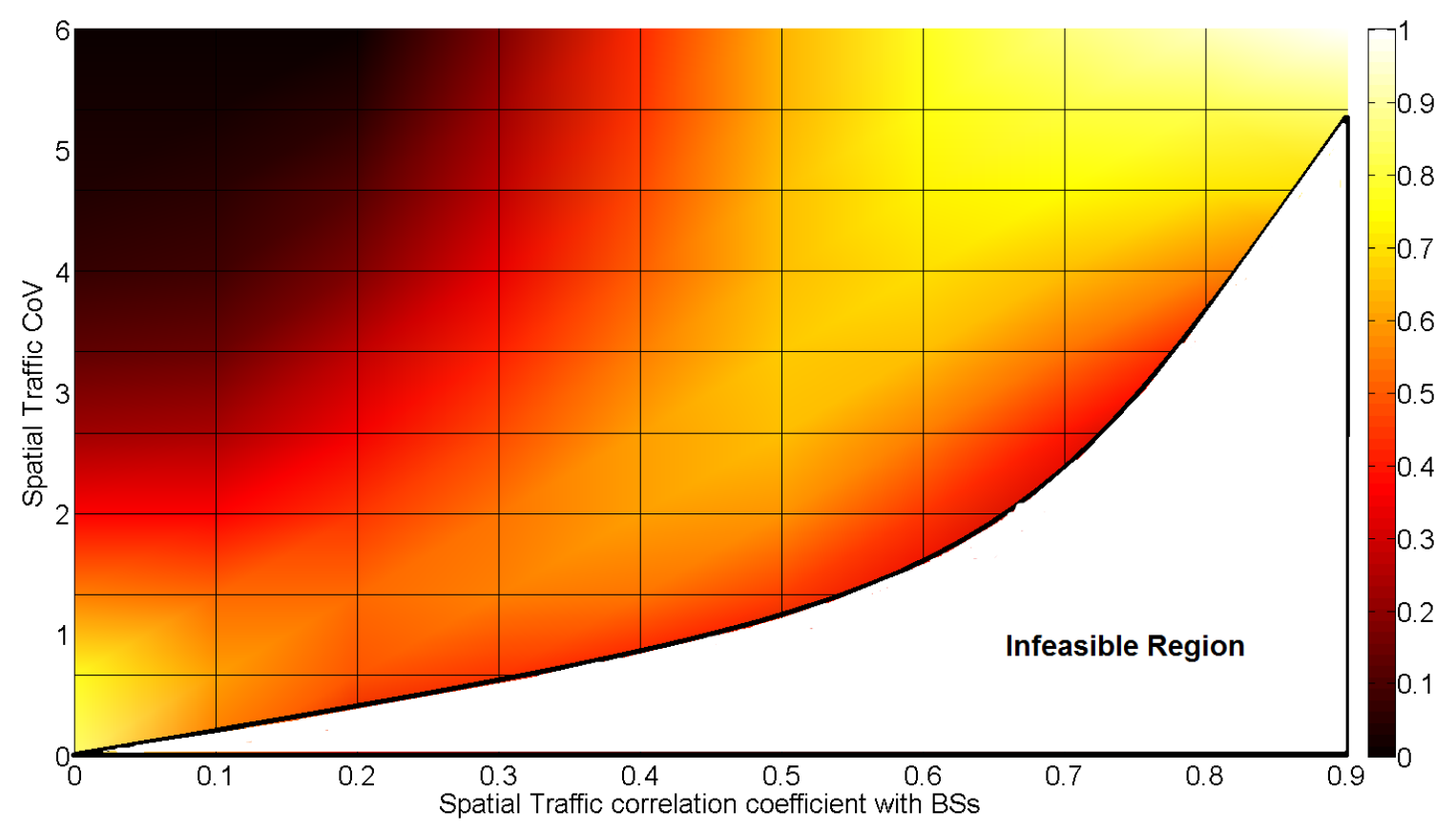

Figure 17: The coverage probability with minimum SINR threshold of $10 \mathrm{~dB}$ is shown for the feasible normalized $\mathrm{CoV}$ and correlation coefficient region.

The following are the observations made from Figures 16 and 17:

- As the correlation coefficient between UE and BS locations increases, the mean user rate and coverage probability also increase as expected, due to the fact that the received signal strength from the serving BS is increased and that from the interfering BSs is decreased. As a result, the SINR statistics are improved.

- For independent UE and BS locations (or, for low correlation coefficient values), as $\mathrm{CoV}$ increases, the mean user rate and coverage probability decrease. This is due to the fact that as CoV increases, the UEs become concentrated in some localities, and as a result, some BSs don't have any UEs connected to them, i.e., the resources of these BSs are wasted. On the other hand, some BSs are overloaded with more than the average number of UEs which have to share the available resources.

- For correlated UE and BS locations (high correlation coefficient values), as CoV increases, the mean user rate and coverage probability also increase. In these 
scenarios, with increasing CoV, UEs move more and more towards the BSs (cell centers); as a result, the SINR statistics are improved.

\subsection{Conclusion}

In this chapter, a new statistical approach for modeling the spatial traffic in heterogeneous cellular networks is introduced. A number of novel distance-based traffic measures in space are proposed which can be considered as the equivalents of the iat in the time domain. Only two statistical parameters are used to regulate the heterogeneity and bias of traffic towards the BSs. Stochastic geometry is used to generate realistic and adjustable traffic. The effects of the realistic traffic modeling on the performance of heterogeneous wireless cellular networks is illustrated.

The traffic modeling method presented in this chapter is general enough to be applied in other wireless networks as well. For instance, the relaying and routing problems in ad hoc and Wi-Fi networks are closely related, and are dependent on the distribution of the network terminals. In wireless networks, the cell switch-off framework is also highly dependent on the spatial distribution of the traffic.

It is interesting to note that various characteristics of the user distributions in wireless networks can be observed in other phenomena as well. For instance, the similar problems of heterogeneity of distribution or self-similarity arise in micro-cosmic level investigations in chemistry and particle physics and in macro-cosmic level studies in astrophysics. 


\section{Chapter 3}

\section{Analytic Modeling of HetHetNets}

\subsection{Introduction}

In the future $5 \mathrm{G}$ wireless networks where every traffic hot-spot is envisioned to be equipped with a base station (BS), the user equipments (UEs) are more likely to be biased towards and collocated with BSs (instead of being uniformly distributed). However, the most common approach in the recent literature in the spatial modeling and analysis of the wireless cellular networks is the use of two independent homogeneous Poisson point processes (PPPs) for the locations of UEs and BSs [18], mainly, for the sake of analytical tractability. Recently, in wireless modeling literature, e.g., in [28] and [47], heterogeneous spatial traffic modeling in cellular networks has been investigated. However, to the best of the authors' knowledge, there is no analytical study in the literature.

The contribution of this chapter is to study the statistical characteristics, i.e., the probability distribution function, of the signal-to-interference ratio (SIR) in the downlink of wireless cellular networks with non-uniform spatial traffic distribution. We use a smoothly adjustable model for modeling heterogeneous spatial traffic distribution in wireless cellular networks. In the proposed model, the correlation between the UEs and BSs is tunable and the distribution of UEs is adjustable between two extreme cases, i.e., the uniform PPP distribution and the totally correlated scenarios (Section 3.2). Consequently, closed-form expressions for the lower-bounds and the upper-bounds of the SIR (and coverage) probabilities are drawn (Section 3.3). It is shown via Monte-Carlo simulation that the bounds are reasonably tight and these bounds can be used as estimators of the network performance metrics (Section 3.4). Some remarks and future work directions are given in Section 3.5. 


\subsection{Proposed Non-Uniform UE Modeling}

We study a simplified model of the downlink of a wireless cellular network comprising of macro-BSs with omni-directional antennas. The BSs are distributed by a uniform $\operatorname{PPP} \Phi_{B}$ with density $\lambda_{B}$. The transmit power $P_{t}$ at all BSs is fixed and the distance dependent attenuation between a typical UE and a BS $b$ located $d_{b}$ meters away is denoted by $L_{b}=10 \alpha \log _{10}\left(d_{b}\right)$, where $\alpha>2$ is the path-loss exponent. The multipath fading is not considered in this study. The shadow fading in the channel (in the logarithmic domain), $h_{b}$, is modeled by an i.i.d. Gaussian distribution with 0$\mathrm{dB}$ mean and $\sigma$-dB standard deviation, i.e., $h_{b} \sim N(0, \sigma)$. Hence, assuming that the antenna gains are included in the transmission power, the received power at a typical UE from BS $b_{i}$ is $P_{b_{i}}=P_{t}-L_{b_{i}}+h_{b_{i}}$. We consider a dense network which is interference-limited; therefore, in this chapter, we assume that the noise power is negligible. We denote the BS that serves the UE as BS $s$; then, the aggregate SIR (in linear domain) can be written as,

$$
I_{a g g}=\sum_{b_{i} \in \Phi_{B} \backslash s} P_{b_{i}}
$$

and

$$
\gamma_{l i n}=\frac{P_{s}}{I_{a g g}}
$$

respectively. Therefore, in $\mathrm{dB}$ domain, SIR can be stated as

$$
\gamma[\mathrm{dB}]=P_{s}[\mathrm{dBm}]-I_{a g g}[\mathrm{dBm}]
$$

We assume that each UE is associated with the closest BS. The spectral efficiency (SE) can be stated as $\eta=\log _{2}(1+\gamma)$ using the Shannon's formula. We presume equal resource scheduling. UE temporal traffic model is assumed to be full-buffer and best-effort.

Starting with a uniform PPP, we move every UE closer to its serving BS. The distances from the UE to its serving $\mathrm{BS}\left(B S_{1}\right.$ or $\left.s\right)$ and to its second closest (dominant interferer) BS ( $B S_{2}$ or $I$ ) are denoted as $R_{1}$ (or $\left.d_{s}\right)$ and $R_{2}$ (or $\left.d_{I}\right)$, respectively. After moving the UE closer to $B S_{1}$, the new distances are denoted as $R_{1}^{\prime}$ and $R_{2}^{\prime}$. A sample scenario is shown in Figure 18. Since we start with the PPP, any move of the already dropped UEs towards their serving BSs would increase the correlation to the BSs and 


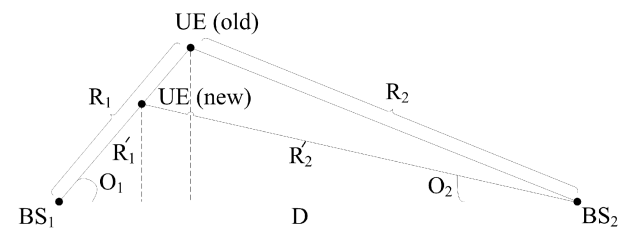

Figure 18: Starting with a PPP, every UE is moved closer to its serving BS.

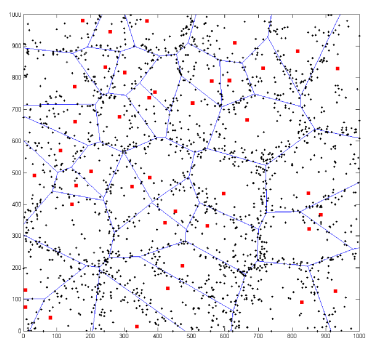

(a) $n=0.5$

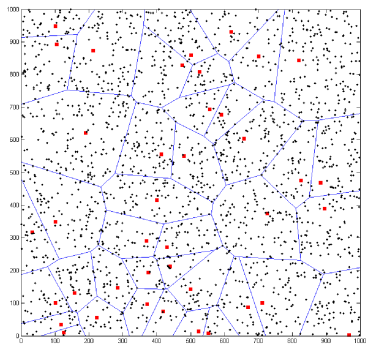

(b) $n=1$ (i.e., PPP)

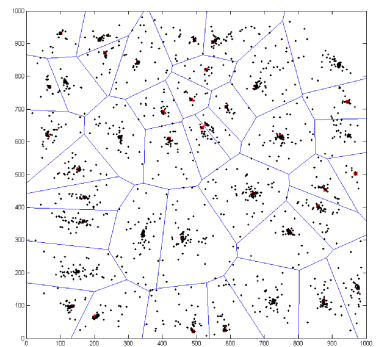

(c) $n=5$

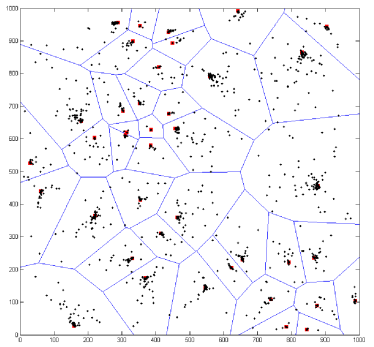

(d) $n=10$

Figure 19: The red squares denote the BSs and the blue lines represent their associated Voronoi tessellation. Small black dots denote the UEs.

also the clustering effect in distribution. For the reasons that will become clear soon, in our model, we move the UE to the new location so that

$$
\frac{R_{1}^{\prime}}{R_{2}^{\prime}}=\left(\frac{R_{1}}{R_{2}}\right)^{n}
$$

where $n$ determines the correlation between UEs and BSs. Since $R_{1} \leqslant R_{2}$, for $n>1$ the UE moves towards the serving BS resulting in a more clustered and BS-correlated pattern, and for $0<n<1$ the UE moves towards the cell-edges. The $n=1$ case represents the traditional uniform PPP distribution. In Appendix C, we explain how we can calculate the new location of UEs and move the UEs in order to generate the desired patterns. Figure 19 shows some sample patterns with associated $n$ values in a wireless cellular network. 


\subsection{Analytical Modeling}

For a homogeneous PPP, the SIR and an upper-bound on it can be expressed as

$$
\gamma=P_{s}-I_{a g g} \leqslant \hat{\gamma}=P_{s}-P_{I}=P_{t}-L_{s}+h_{s}-\left(P_{t}-L_{I}+h_{I}\right)
$$

where $\hat{\gamma}$ is an upper-bound on the SIR, $h_{s} \sim N(0, \sigma)$, and $h_{I} \sim N(0, \sigma)$. In (22)

we use the fact that the aggregate interference power $I_{a g g}$ is always greater than the power of the dominant interferer alone $P_{I}$. Therefore, the SIR can be upper-bounded as

$$
\gamma \leqslant \hat{\gamma}=L_{s}-L_{I}+h
$$

where $h \sim N\left(0, \sigma^{\prime}\right)$ is the summation of two independent Gaussian variables and $\sigma^{\prime}=\sqrt{2} \sigma$. Using the path-loss equation in Section 3.2, $\hat{\gamma}$ is formulated as

$$
\hat{\gamma}=10 \log _{10}\left(d_{s}^{-\alpha}\right)-10 \log _{10}\left(d_{I}^{-\alpha}\right)+h=10 \cdot \alpha \cdot\left(\frac{\log \left(d_{I}\right)}{\log (10)}-\frac{\log \left(d_{s}\right)}{\log (10)}\right)+h
$$

The upper-bound, $\hat{\gamma}$, can be simplified as

$$
\hat{\gamma}=\frac{10}{\log (10)} \cdot \alpha \cdot \log \left(\frac{d_{I}}{d_{s}}\right)+h=-\frac{10}{\log (10)} \cdot \alpha \cdot \log \left(\frac{d_{s}}{d_{I}}\right)+h
$$

Substituting $d_{s} / d_{I}$ with $R$ we have

$$
\hat{\gamma}=-\frac{10}{\log (10)} \cdot \alpha \cdot \log (R)+h
$$

Since $0 \leqslant d_{s} \leqslant d_{I}, R \in[0,1]$.

Lemma 2. In PPP scenarios (where BSs and UEs are distributed by independent PPPs), $R$ has the following cumulative distribution function:

$$
F_{R}(r)=p(R \leqslant r)=r^{2}
$$

\section{Proof. See Appendix B.}

Using Lemma 2 and the inverse transform theorem [70], it can be shown that the random variable $U=R^{2}$ has uniform distribution. Substituting $R$ with $U^{0.5}, \hat{\gamma}$ is 
expressed as

$$
\widehat{\gamma}=-\frac{10}{\log (10)} \cdot \alpha \cdot \log \left(U^{0.5}\right)+h=-\frac{10}{2 \log (10)} \cdot \alpha \cdot \log (U)+h
$$

where $U$ has standard uniform distribution. Further, again from the inverse transform theorem, we know that the negative logarithm function of a standard uniform random variable has exponential distribution with parameter 1 . Therefore, substituting $X=$ $-\log (U)$,

$$
\hat{\gamma}=\frac{10}{2 \log (10)} \cdot \alpha \cdot X+h
$$

where $X \sim \exp (1)$.

Finally, the SIR is estimated as

$$
\hat{\gamma} \sim \exp \left(\frac{10}{2 \log (10)} \cdot \alpha\right)+N\left(0, \sigma^{\prime}\right) \sim \operatorname{EMG}\left(0, \sigma^{\prime}, \frac{10}{2 \log (10)} \cdot \alpha\right)
$$

where EMG is the well-known exponentially modified Gaussian random variable [74].

Having the statistical distribution of SIR, we can calculate the coverage probability with a threshold $\theta$ as

$$
P_{c}(\theta)=p(\hat{\gamma}>\theta)=1-F_{\hat{\gamma}}(\theta)
$$

Now we can consider a non-uniform UE pattern which is achieved by applying the modeling approach explained in Section 3.2. In this pattern, starting from a PPP pattern, every UE is moved so (21) holds. Therefore, for each UE, the following holds:

$$
R^{\prime}=\frac{R_{1}^{\prime}}{R_{2}^{\prime}}=R^{n}
$$

Therefore, following the same procedure as PPP, we can substitute the new $R^{\prime}$ variable with the $n$-th power of the old $R$ leading to the following statement:

$$
\hat{\gamma}=-\frac{10}{\log (10)} \cdot \alpha \cdot \log \left(R^{n}\right)+h=-\frac{10}{\log (10)} \cdot \alpha \cdot n \cdot \log (R)+h .
$$

Then, substituting $R$ with $U^{0.5}$, we get

$$
\widehat{\gamma}=-\frac{10}{2 \log (10)} \cdot \alpha \cdot n \cdot \log (U)+h,
$$


where $U$ has standard uniform distribution. Therefore,

$$
\widehat{\gamma}=\frac{10}{2 \log (10)} \cdot \alpha \cdot n \cdot X+h
$$

where $X \sim \exp (1)$. Finally, we have

$$
\hat{\gamma} \sim \exp \left(\frac{10}{2 \log (10)} \cdot \alpha \cdot n\right)+N\left(0, \sigma^{\prime}\right) \sim \operatorname{EMG}\left(0, \sigma^{\prime}, \frac{10}{2 \log (10)} \cdot \alpha \cdot n\right)
$$

In modeling wireless cellular networks with PPP distribution of BSs, for the sake of tractability, the number of BS points which are distributed in the field is assumed to be infinite. However, the real number of BSs is normally limited. Therefore, the amount of interference in infinite modeling is over-estimated. Therefore,

$$
\gamma=P_{s}-I_{a g g} \geqslant \check{\gamma}=P_{s}-I_{\infty}
$$

where $I_{\infty}$ is the interference power when there are infinitely many BSs and $\check{\gamma}$ is a lower-bound on the SIR.

In order to achieve a closed-form expression for $\check{\gamma}$, in this chapter, we assume that the aggregate interference in an infinite network can be stated as a coefficient multiplied by the strongest (dominant) interference, i.e.,

$$
I_{\infty}=\beta \cdot P_{I},
$$

where $\beta$ is a coefficient and $P_{I}$ is the power of the dominant interfering BS. The coefficient $\beta$ can be calculated as the expected value of the ratio of the total interference to the strongest interference. Therefore,

$$
\beta=\mathbb{E}\left(\frac{P_{\infty}}{P_{I}}\right)=\mathbb{E}\left(\frac{P_{I}+P_{2}+P_{3}+\ldots}{P_{I}}\right)=\mathbb{E}\left(\frac{d_{I}^{-\alpha}+d_{2}^{-\alpha}+d_{3}^{-\alpha}+\ldots}{d_{I}^{-\alpha}}\right),
$$

where $P_{i}$ is the interference power from the $i$-th strongest interferer and $d_{i}$ is the distance from the typical UE to the $i$-th interferer. Therefore,

$$
\beta=\mathbb{E}\left(1+\left(\frac{d_{I}}{d_{2}}\right)^{\alpha}+\left(\frac{d_{I}}{d_{3}}\right)^{\alpha}+\left(\frac{d_{I}}{d_{4}}\right)^{\alpha}+\ldots\right)
$$

It is shown in [75] that in PPP scenarios, the second power of the distance to $k_{t h}$ 
neighbor has Erlang distribution with mean $k \cdot \lambda$ where $\lambda$ is the density of the PPP process. Therefore,

$$
\beta=\mathbb{E}\left(1+\left(\frac{2}{3}\right)^{\alpha / 2}+\left(\frac{2}{4}\right)^{\alpha / 2}+\left(\frac{2}{5}\right)^{\alpha / 2}+\ldots\right)
$$

which can be expressed as

$$
\beta=\mathbb{E}\left(2^{\alpha / 2}\left(\left(\frac{1}{2}\right)^{\alpha / 2}+\left(\frac{1}{3}\right)^{\alpha / 2}+\left(\frac{1}{4}\right)^{\alpha / 2}+\ldots\right)\right) .
$$

Using the Riemann Zeta Function, $\beta$ can be written as

$$
\beta \approx 2^{\alpha / 2}(\zeta(\alpha / 2)-1)
$$

Therefore, SIR in homogeneous scenarios can be bounded as follows:

$$
\begin{array}{r}
\check{\gamma} \sim \operatorname{EMG}\left(0, \sigma^{\prime}, \frac{10}{2 \log (10)} \cdot \alpha\right)-10 \cdot \log _{10}\left(2^{\alpha / 2}(\zeta(\alpha / 2)-1)\right) \leqslant \\
\gamma \leqslant \hat{\gamma} \sim \operatorname{EMG}\left(0, \sigma^{\prime}, \frac{10}{2 \log (10)} \cdot \alpha\right) .
\end{array}
$$

Using the same procedure which we followed in deriving the upper-bound in heterogeneous cases, we can derive a lower-bound estimate for SIR in cellular networks with heterogeneous UE distribution. Therefore, the lower-bound and upper-bound on the SIR can be expressed as

$$
\begin{array}{r}
\check{\gamma} \sim \operatorname{EMG}\left(0, \sigma^{\prime}, \frac{10}{2 \log (10)} \cdot n \cdot \alpha\right)-10 \cdot \log _{10}\left(2^{\alpha / 2}(\zeta(\alpha / 2)-1)\right) \leqslant \\
\gamma \leqslant \hat{\gamma} \sim \operatorname{EMG}\left(0, \sigma^{\prime}, \frac{10}{2 \log (10)} \cdot n \cdot \alpha\right)
\end{array}
$$

\subsection{Simulation Results}

This section presents the simulation setup and results. In our simulations, the spatial density of UEs and BSs is $10^{-3} / \mathrm{m}^{2}$ and $5.0 \times 10^{-5} / \mathrm{m}^{2}$, respectively. The total BS transmit power is $30 \mathrm{dBm}$ and path-loss exponent is 4 . First, we captured the CDF of SIR in various scenarios with different $n$ values. Figure 20 shows the results. As seen in Fig. 20, as $n$ increases, the distribution of SIR tends to higher values. Moreover, it is illustrated that the lower-bound and the upper-bounds presented in Section 3.3 


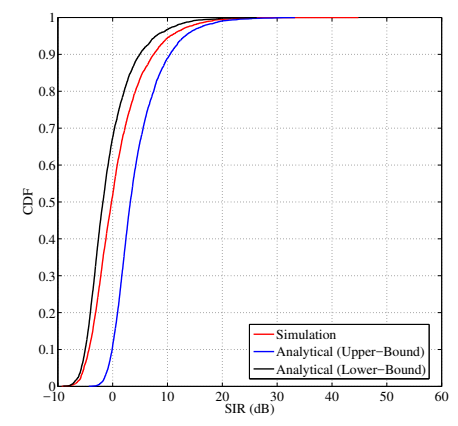

(a) $n=0.5$

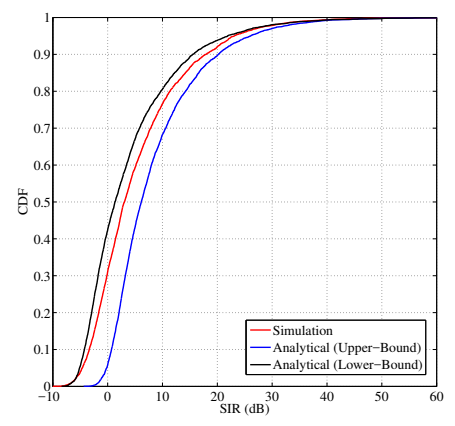

(b) $n=1$ (i.e., PPP)

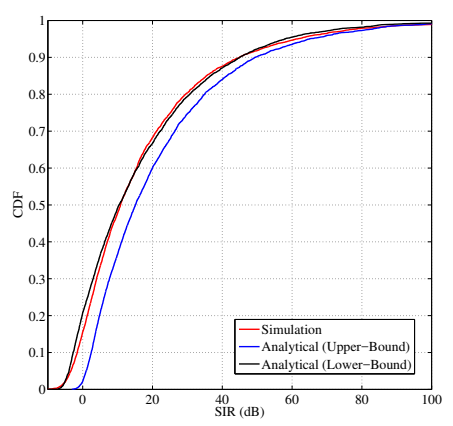

(c) $n=3$

Figure 20: The CDF of SIR is illustrated. With increase in $n$ value, the distribution of SIR tends to higher values.

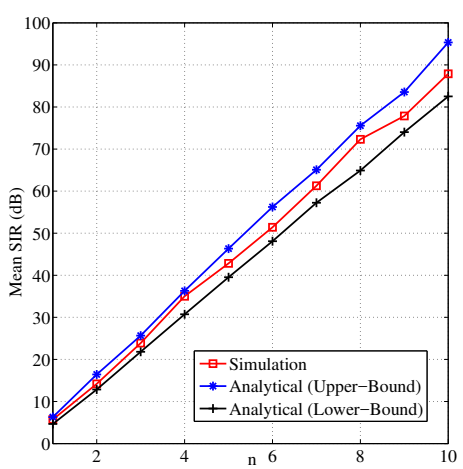

Figure 21: Mean UE SIR for different $n$ values is shown.

are very tight, i.e., in the worst case scenarios the difference between the analytical bounds and the simulation results is around $5 \mathrm{~dB}$. The next performance metric is the mean SIR over the entire network. Mean UE SIR for different $n$ values is presented in Fig. 21. It is shown that the relation between the $n$ value and the mean SIR is a linear relation, as suggested by (35).

\subsection{Conclusion}

In this chapter, we derived closed-form analytical models for SIR and coverage in wireless cellular networks with heterogeneous traffic. This work can be extended in many directions. First, other modeling approaches might be presented which result in 
more accurate expressions. Moreover, closed-form expressions for other metrics such as UE rates could be derived. 


\section{Chapter 4}

\section{UIL in HetHetNets with Backhaul Capacity Constraints}

\subsection{Introduction}

In this chapter, we propose a novel modeling approach for heterogeneous networks with heterogeneous spatial traffic distribution (HetHetNets). Specifically, in the proposed model, a particular ratio of UEs are collocated with the BSs while the rest of UEs are independently and homogeneously distributed in the network. Moreover, the proposed model presumes backhaul connections with constrained capacity. We study the impact of this more realistic network modeling on the effectiveness of the spatial user-in-the-loop (UIL) schemes in HetHetNets. Spatial UIL assumes that (some) UEs can be influenced by the operator to move in the network. Finally, we propose a new objective for the UIL mechanism that takes into account the impact of the BS loads and the backhaul capacities on the network performance.

The spatial distribution of wireless user equipments (UEs), the wireless access network, and the backhaul network can be viewed as three layers of Heterogeneous wireless cellular Networks with Heterogeneous traffic distribution (HetHetNets). The spatial distribution of UEs constitutes the network traffic demand characteristic. On the other hand, the access connection between UEs and the base stations (BSs), and the backhaul connection between the BSs and the core IP network constitute the network supply capacity.

The distributions of the wireless traffic demand and the wireless capacity supply don't necessarily match in the wireless cellular networks, neither in the time domain nor in the space domain. Commonly, some locations (times) of the network are 
overloaded while other locations (times) are underloaded. The problem with this lack of match is twofold: 1) In the overloaded parts of the network, the available resources must be shared to serve excessive amounts of demand, which leads to low data rates per user. 2) In the underloaded parts of the network, the unused resources are wasted. The root cause to this problem is that the idle network capacity and idle resources cannot be reserved in underloaded locations (times) to be transferred to the overloaded locations (times).

In a wireless cellular network with ideal backhaul connections, the lack of capacity is mainly due to the access connection limitation, i.e., wireless access problem and low channel quality between UEs and BSs. However, in the envisioned $5 \mathrm{G}$ networks with small-cells deployed in residential and office buildings, the assumption of ideal backhaul is more optimistic than realistic; especially considering the fact that a main portion of the small-cells, i.e., femto-cells, will be deployed by the customers rather than the service providers. Recently, there has been an increasing interest in the literature for the study of limited backhaul capacities in future 5G HetNets [76].

Figure 22 illustrates an example HetHetNet comprising of macro-cells, pico-cells, and femto-cells with different backhaul capacities. In the networks with limited backhaul capacity, the UE rate limit will be a consequence of limited backhaul in addition to the wireless access connection limit. The backhaul connections in this chapter are assumed to be wired. However, the proposed model in this chapter is applicable to HetHetNets with wireless backhaul connections.

One solution to the problem of mismatch between supply distribution and demand distribution is to bring the wireless traffic demand and wireless capacity supply together in the time domain and the space domain. Predictive resource management and user location tracking [29], and optimized small-cell BS deployment in traffic hotspots, are among mechanisms which try to bring wireless capacity supply to wireless traffic demand location and time. On the other hand, user-in-the-loop (UIL) [9], defined below, is a relatively new and significantly effective method of bringing traffic demand to the time and location of wireless capacity supply (traffic shaping).

The UIL concept aims at influencing the user behavior (which can be viewed as the 'layer-8' in OSI network models) in a wireless network in order to obtain a better spectral efficiency by convincing the users to move from one location to a better one or to avoid traffic congestion by postponing session traffic out of the busy hours. Indeed, UIL extends the past assumption of the user being a traffic generating and 


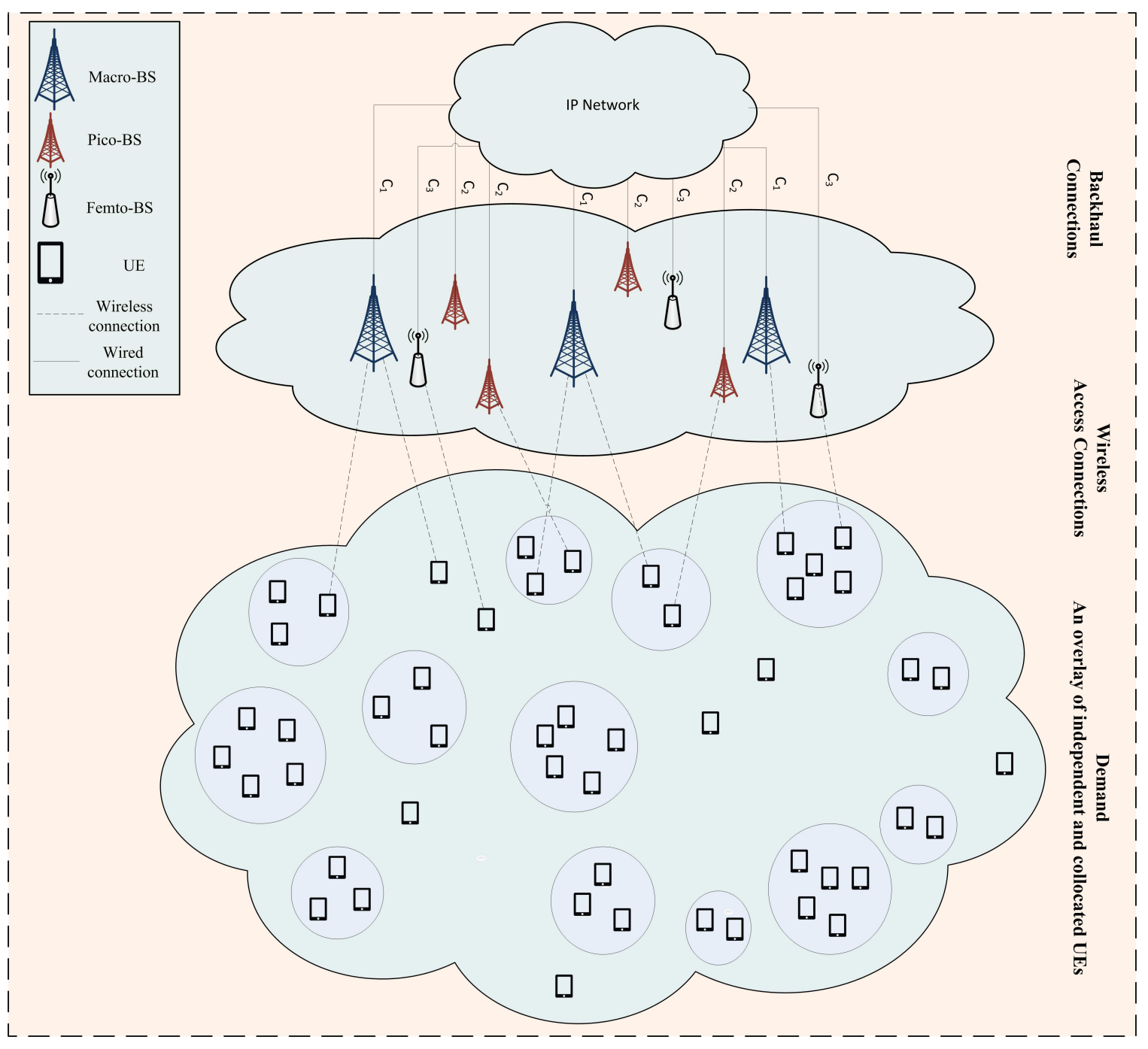

Figure 22: In the envisioned 5G HetHetNets, the assumption of backhaul connections with unlimited capacity is very optimistic. Specially, the envisioned femto-cells and pico-cells might have backhaul connections with constrained capacity. In this figure, a sample 3-tier HetHetNet with macro-cells, pico-cells and femto-cells, which have different backhaul capacities, is demonstrated. 
consuming black box only (in nature similar to the noise input into a system). Instead, the system-theoretic framework allows a control input into the user block, on which the user receives suggestions and incentives (and eventually penalties) in order to convince him to diverge from the default behavior (which is uncontrolled, i.e., open loop), so that the traffic can be shaped [9].

Indeed, complementing the engineering for the growth of the supply side, the engineering for the control of the demand side is referred to as UIL and therefore motivated here. UIL proposes 'dynamic pricing' based on user behavior and his willingness to adapt with network situations, compared to current 'static pricing' policies. Results from a survey, which measures how willing a user is to respond to such control, are also presented in [9]. Section 4.3 describes the UIL in details.

In order to study the performance of wireless cellular networks under heterogeneous traffic distribution and limited backhaul assumption, and to capture and measure the impact of various methods, such as UIL, on the network performance, the first step is to model the traffic demand and capacity supply in the time domain and the space domain.

Traffic demand modeling in the time domain has been well-investigated in the literature. Traditionally, in voice-only networks, homogeneous Poisson process models were accurate enough to model traffic in time. After the emergence of different applications, such as video and data with variable data rate demands, the Poisson model failed to capture the traffic statistics; as a result, various heterogeneous (super-Poisson) traffic models based on the hidden Markov model (HMM), Markov modulated Poisson process (MMPP) [11], and other stochastic methods have been proposed in the literature and used for performance analysis.

Similarly, in the space domain, a popular approach in modeling and analysis of the supply and demand in HetNets has been the use of two independent Poisson point processes (PPPs) for the locations of BSs and UEs [4]. Despite analytical tractability, this popular approach has a major shortcoming: although the PPP model may be a fitting one for the BS locations, it is less adequate for the UE locations, mainly due to the fact that the model is not adjustable (tunable) to represent the amount of heterogeneity (non-uniformity) in the UE locations. There is still relatively little literature that takes into account the heterogeneous spatial distribution of the traffic demand in wireless cellular networks [22, 26, 28].

The goals and the main contributions of this chapter are summarized as follows: 


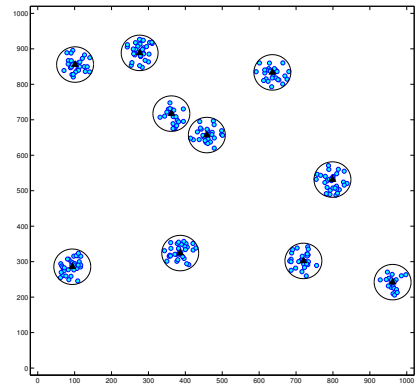

(a) $\mathrm{p}=0$ (i.e., pure Matern), $\mathrm{CoV}=5.47$

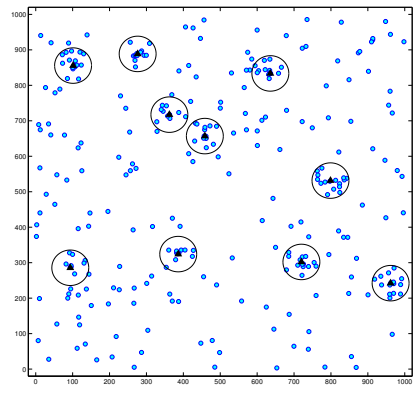

(d) $\mathrm{p}=0.6, \mathrm{CoV}=3.27$

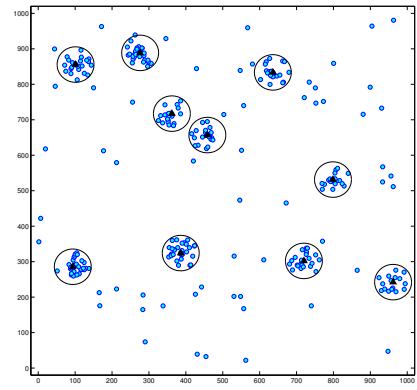

(b) $\mathrm{p}=0.2, \mathrm{CoV}=4.79$

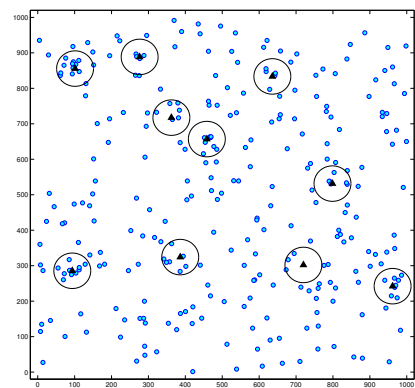

(e) $\mathrm{p}=0.8, \mathrm{CoV}=2.16$

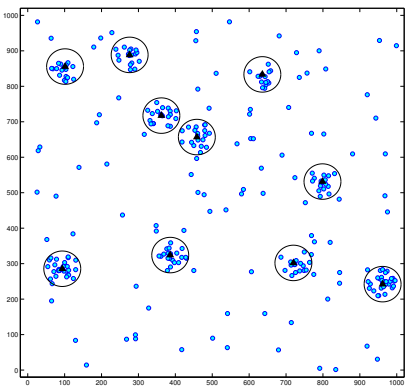

(c) $\mathrm{p}=0.4, \mathrm{CoV}=4.04$

Figure 23: Various UE distributions with different clustering properties are illustrated in a $1000 \mathrm{~m} \times 1000 \mathrm{~m}$ network. Black triangles denote the BSs and small dots denote the UEs. Starting from a pure Matern point process (a), the ratio of independent UEs increases and the CoV decreases until a homogeneous PPP (f) with no clusters is reached with $\mathrm{CoV}=1$. The number of BSs is 10 and the Matern cluster radius is assumed to be 50 meters. 
- We introduce a novel HetHetNet modeling, i.e., an adaptive spatial traffic model with adjustable heterogeneity to capture the characteristics of wireless demand distribution in wireless heterogeneous cellular networks.

- We study the impact of more realistic heterogeneous (non-uniform) spatial traffic demand distribution on the efficiency of the UIL method in HetHetNets with backhaul capacity limitation.

- We propose a new objective for the UIL mechanism that takes into account the impact of the BS load and the backhaul capacity on the network performance.

The remainder of this chapter is organized as follows. In Section 4.2, the spatial traffic modeling and the network modeling are described. The basic UIL is reviewed in Section 4.3. The new UIL method involving BS load and backhaul capacity is introduced in Section 4.4. In Section 4.5, the simulation parameters and results are presented, and Section 4.6 presents conclusions and remarks.

\subsection{Modeling HetHetNets}

Although PPP modeling leads to analytical tractability [77, 78], a more realistic model results in more accurate understanding of network performance. The concept of user distribution is directly related to the network performance in wireless networks in general and in wireless cellular networks in specific. There are two main aspects of experienced rate which are affected by user distribution: the load on BSs which is determined by the clustering properties of user distribution, and the spectral efficiency of UEs which is a function of signal-to-interference-plus-noise (SINR) which is, in turn, a function of distance between UEs and BSs. In this section, we propose a novel wireless cellular network modeling approach which has the following properties:

1. The spatial distribution of wireless traffic demand (active UEs) is not homogeneous (uniform) but adjustable and heterogeneous (nonuniform).

2. The capacity of backhaul connections is not unlimited (infinite) but constrained (finite). 


\subsubsection{UE Distribution Model}

While homogeneous PPP assumption for the UE distribution is not realistic, highly clustered distributions with no standalone UEs is also unrealistic. The requirement is an adjustable model which is tunable (preferably with few parameters) to model a wide range of scenarios from highly homogeneous PPP to highly heterogeneous clustered scenarios.

To generate such an adjustable and heterogeneous spatial traffic demand, we should first note that the wireless devices, i.e., UEs, can be divided into two different categories: 1) the wireless devices which are correlated (collocated) with social points of interest, i.e., residential and office buildings, bus stations, shopping malls, and so forth, and 2) the wireless devices which are not attracted around the points of interest, i.e., the wireless devices which are independent from the points of interest. We assume that the set of points of interest is the same as the set of BSs, i.e., all points of interest are equipped with BSs. Sample scenarios are shown in Figure 23.

The proposed user distribution model in this chapter is as follows: $N_{u}$ active user devices are generated while $N_{u}$ follows a Poisson distribution with mean $\lambda_{u} \times A$ $\left(N_{u} \sim \operatorname{Poisson}\left(\lambda_{u} \times A\right)\right)$ where $A$ is the total network area. Every new user is labeled as BS-independent with a probability of $p$ or BS-correlated with a probability of $1-p$. The BS-independent devices are distributed uniformly by a homogeneous Poisson process, while the BS-correlated points are located uniformly in a ball centered at one of the points of interest. The points of interest might have different weights attracting devices with different probabilities. In this chapter, we assume that all of the points of interest have equal weight.

The real world traffic distribution is very similar to the described model. Consider a snapshot of user locations in a cellular network (for instance, a campus snapshot). Users can be divided into two main categories. The first category includes the nomadic users who are inside or around buildings or other points of interest. The second category is the mobile users moving from one building to another, or from one point of interest to another. We don't claim that this model can fit to any scenario of user distribution but this model covers a majority of users and can be adjusted to various scenarios with limited number of parameters.

In particular, the distribution of UEs is an overlay (a superposition) of two independent distributions. The first one is a PPP with density $p \lambda_{u}$ and the second one is a Matern point process [58] with total density of $(1-p) \lambda_{u}$. In Matern point processes, 
first, a parent process of cluster-heads are distributed uniformly in a region, then, cluster members are distributed uniformly in circles around the cluster-heads.

In a network scenario with $p=0$, all devices are located around points of interest and the resulting pattern is a clustered Matern point process. On the contrary, a point pattern generated with $p=1$ is totally random, i.e., traditional uniform PPP. Any other value of $p$ results in an overlay pattern with superposition of a Poisson point process and a Matern point process (Figure 23).

A very important problem in heterogeneous UE modeling is the measurement of the level of the heterogeneity. It is crucial to have a benchmark metric which determines the heterogeneity of the distribution with one (or few) parameter(s), so it is easy for everybody to gain an understanding of the level of clustering of UEs by having this metric value. In [26], we proposed simple metrics for the measurement and understanding of the heterogeneity of spatial point patterns. Specifically, we proposed the statistical characteristics of the random tessellations of point distributions as accurate and very appropriate metrics of spatial heterogeneity. We showed that the coefficient of variation $(\mathrm{CoV})$, defined as the standard deviation $\sigma$ divided by mean $\mu,\left(\frac{\sigma}{\mu}\right)$, is a revealing statistical parameter which captures a majority of the statistical characteristics of a metric. We also showed that the geometrical inferences of conventional tessellations such as Voronoi tessellations and Delaunay tessellations are very promising candidates. Such metrics as Voronoi-cell-areas and Delaunay-celledge-lengths are shown to be accurate enough and can be considered as analogues of popular inter-arrival-times metric in the temporal traffic modeling.

A CoV value of 0 means that the UEs are organized in a very structured manner and the Voronoi cells and Delaunay cells are all equal. A CoV value of 1 is associated with a completely random distribution where UEs are PPP distributed. CoV values between 0 and 1 refer to sub-Poissonian distributions in which the distribution is more homogeneous than Poisson. Finally, a CoV value of more than 1 means that the points are distributed heterogeneously (or non-uniformly) compared to PPP (superPoissonian).

This gives the opportunity of unified and non-parameterized traffic modeling and measurement in time domain and space domain. Indeed, a parameter such as $p$ in our model in this chapter, is an internal parameter of the traffic generation process while $\mathrm{CoV}$ is a benchmark parameter that can be used to compare the heterogeneity of different patterns. 


\subsubsection{System Model}

We model the downlink of a heterogeneous wireless cellular network with heterogeneous traffic distribution (HetHetNet) comprised of $K$ tiers of BSs (e.g., macro, pico, and femto) with omni-directional antennas. BSs of tier $k$ have transmission power of $P_{k}$, backhaul capacity of $C_{k}$ and are spatially distributed as a PPP $\Phi_{k}$ of spatial density $\lambda_{k}$. The BSs of different tiers of the network are distributed independently.

The fading (power) between a BS and a UE is assumed to be i.i.d exponential (Rayleigh fading). The standard path loss function is given by a simple model reciprocal to the $\alpha$-power of distance where $\alpha>2$ is the path loss exponent. Hence, assuming that the antenna gains are included in the transmission power, the received power at a typical UE from a BS belonging to tier $i$ is the product of transmit power at that tier by the power loss. Assuming that the UE connects to this BS, the resulting aggregate interference and the SINR expression can be calculated accordingly. We assume that each UE connects to the BS whose average signal power, hence average SINR, is the strongest. The spectral efficiency (SE) can be stated as $\eta_{x}=\log _{2}\left(1+\gamma_{x}\right)$ using the Shannon formula. Assuming equal resource scheduling, the user rate can be calculated as $R_{u}=\min \left(1, C_{a_{u}} / L_{a_{u}}\right) \times \eta_{x_{u}} W N_{a_{u}}^{-1}$ where $W$ is the total BS bandwidth,

$a_{u} \in \bigcup_{k=1}^{K} \Phi_{k}$ is the BS associated to $u, L_{a_{u}}$ is the aggregate load on $a_{u}$ (i.e., the summation of UE loads in an unlimited backhaul scenario which depends on the locations and channel quality of UEs), and $N_{a_{u}}$ is the number of UEs associated with $a_{u}$. The backhaul capacity limits the user rates by a factor of $C_{a_{u}} / L_{a_{u}}$ compared to the unlimited backhaul scenarios. UE temporal traffic model is assumed to be full-buffer and best-effort.

\subsection{Basic UIL}

In this section, we describe the UIL concept and explain the basic UIL schemes which already exist in the literature.

The basic UIL concept aims at controlling the user ('layer-8') behavior in a wireless network in order to obtain a better SE by convincing the users to move from one location to a better one or to avoid traffic congestion by postponing session traffic out of the busy hours. Depending on this impact dimension, the approach is called spatial or temporal UIL control [9]. In both cases the user is within, and part of, a closed-loop control system shown in Fig. 24. In this chapter, we are concerned about 


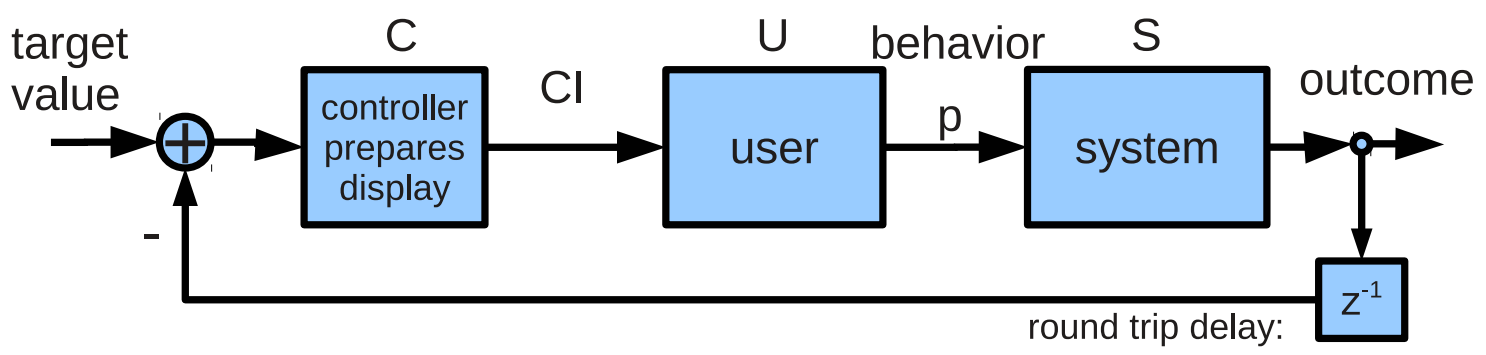

Figure 24: UIL aims at controlling user behavior in a network. User is guided to transmit at a better time or move to a better location. Based on the target behavior and the previous user behavior, the controller decides on the control information (CI) which is displayed in the screen of the user equipment for the user with the associated incentives (or penalties). The user decides 1) to follow the suggestion and deviate from his default behavior in order to receive the offered rewards, or 2) not to follow the suggestion and loose the offered rewards.

spatial UIL.

The user receives suggestions and incentives (or penalties) in order to convince him to diverge from the default behavior. A user within a closed control loop receives this control information (CI) in form of suggestions on the graphical user interface, e.g., a map and directions towards a better location, a better time to start his session (out of the busy hour), or as simple as a color indicator (e.g., green, yellow, red). Before user moves to the new location, all information such as the amount of SE increase, the amount of incentive, and the distance between current and target location are known.

Obviously, the effectiveness of UIL in improving UE experience as well as network throughput depends strongly on the degree of conformance of users regarding the guiding information which, in turn, depends on the incentives which are suggested to the users. For instance, a 20\% discount ignites less motivation in the users to move compared to a $40 \%$ discount. A wise selection of control parameters can lead to increased system throughput as well as network revenue since the increased throughput rises the opportunity for network operators to increase the number of subscribers in general.

The user decides to move or not to move based on the information. The user and its behavior is naturally not subject to a precise science. In order to get some usable 
properties of the input/output system response, two surveys have been conducted in $[79,80]$. A fitting mathematical model derived from the empirical distributions follows an exponential shape for the function between incentive and the probability $p_{d}=\exp (-\beta d)$ of using the service where $d$ is the distance between current user location and suggested location and $\beta$ depends on individuals, incentives, and many other parameters. Therefore, the model can be customized for each user and recorded in central databases. For this thesis, for the sake of simplicity, we assume that the $p_{d}$ function has the same shape ( $\beta$ parameter) for all users. Figure 25 illustrates the probability of movement and the fitting functions based on the surveys conducted in article $[79,80]$.

The main objective of the basic UIL is to suggest to the user the location which maximizes the utility of the user denoted as $U_{u}^{x}=\eta_{x} p_{x}$, where $\eta_{x}$ is the spectral efficiency at location $x$ and $p_{x}$ is the probability of the user moving the distance $d_{x}=\left|x_{u}^{0}-x\right|$ between the current location of the user $x_{u}^{0}$ and the location $x$. The location that gives the best utility, $x_{u}^{*}$, is suggested to the user $u$ and he accepts the suggestion with probability $p_{x}$ and moves to $x_{u}^{*}$ to experience spectral efficiency of $\eta_{x_{u}^{*}}$.

Two different schemes can be used for basic UIL: in the first scheme (Baseline-I), the user is not re-associated to a new BS, therefore, only the locations inside the coverage area of the current serving BS are searched. In the second scheme which is called (Baseline-II), the best SE from all BSs in the network can be searched and if a location with higher SE from another BS is selected and the user accepts to move, the user is re-associated to the new BS.

\subsection{Advanced UIL Schemes}

The user rate $R_{u}$ in a wireless cellular network depends not only on the SE but also on the load and the backhaul capacity of the BS associated with it. Specifically, a UE associated with a highly loaded BS, even with relatively high SINR, has to share the BS resources with other UEs. Moreover, a UE associated with a BS which has limited backhaul capacity, cannot achieve high rate. While in the basic UIL only the spectral efficiency of the users is considered in the utility function, in this section, we 


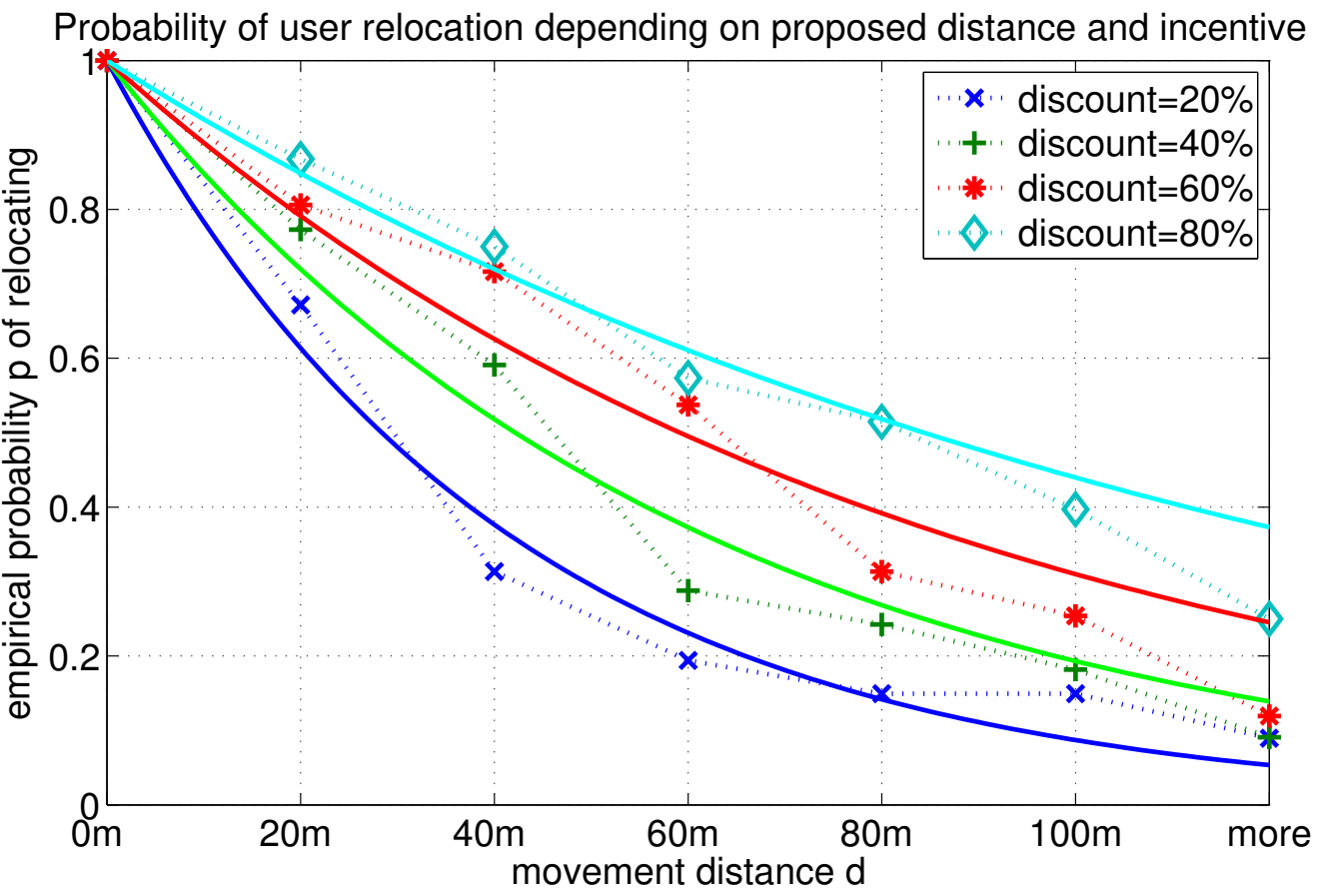

Figure 25: A fitting mathematical model is derived from the empirical probability distributions of using the offered services. The derived model is expressed as an exponential function parameterized by the offered moving distance and the associated incentives (or penalties). 
Table 5: Simulation parameters for Chapter 4

\begin{tabular}{l|l|l}
\hline Parameter & Symbol & Value \\
\hline Spatial density of UEs & $\lambda_{u}$ & $1000 / \mathrm{km}^{2}$ \\
Number of network tiers & $K$ & 3 \\
Spatial density of macro-BSs & $\lambda_{1}$ & $5 / \mathrm{km}^{2}$ \\
Spatial density of pico-BSs & $\lambda_{2}$ & $25 / \mathrm{km}^{2}$ \\
Spatial density of femto-BSs & $\lambda_{3}$ & $25 / \mathrm{km}^{2}$ \\
Total network area & $A$ & $1 \mathrm{~km} \times 1 \mathrm{~km}$ \\
Macro-BS Backhaul capacity & $C_{1}$ & $1 \mathrm{Gbps}$ \\
Pico-BS Backhaul capacity & $C_{2}$ & $100 \mathrm{Mbps}$ \\
Femto-BS Backhaul capacity & $C_{3}$ & $10 \mathrm{Mbps}$ \\
Macro-BS antenna height & - & $25 \mathrm{~m}$ \\
Pico-BS antenna height & - & $10 \mathrm{~m}$ \\
Femto-BS antenna height & - & $10 \mathrm{~m}$ \\
Number of drops & - & 1000 \\
Bandwidth (downlink) & $W$ & $10 \mathrm{MHz}$ \\
Noise power & $\sigma_{n}^{2}$ & $-174 \mathrm{dBm} / \mathrm{Hz}$ \\
Total macro-BS transmit power & $P_{1}$ & $46 \mathrm{dBm}$ \\
Total pico-BS transmit power & $P_{2}$ & $30 \mathrm{dBm}$ \\
Total femto-BS transmit power & $P_{3}$ & $23 \mathrm{dBm}$ \\
Path-loss exponent & $\alpha$ & 3.7 \\
BS and UE antenna gain & - & $0 \mathrm{dBi}$ \\
Time domain traffic model & - & Full buffer \\
Antenna model & - & Omni-directional \\
UE antenna height & $-5 \mathrm{~m}$ \\
Fading model & - & -1 igh fading \\
\hline
\end{tabular}


introduce advanced schemes for UIL which take into account the user rates based on BS load information, backhaul capacity, and SE in the utility function.

In this chapter, we assume that the UEs arrive in the network sequentially. Therefore, the new location is suggested to the newly arrived UEs one by one as time passes. As a result, an optimization framework for the entire network in one shot is not considered. Optimization solutions are suggested as interesting future extensions of this work.

It must be noted that consecutive suggestions to the users to move might be annoying and unacceptable. Therefore, in this thesis, we assume that every user is offered a new location only at the arrival to the network (at the beginning of a session). In an optimization paradigm for UIL, the time and the frequency of performing UIL optimization is an important parameter. If the optimization is done frequently, the users might not accept it and deny to move. On the other hand, if the optimization is done infrequently, many users might complete a full session in an interval without being considered for UIL optimization.

In the UIL scheme, the objective function must be defined in a way that maximizes the rate of a user by suggesting a wise movement to the user so that with minimum possible movement, the user can achieve a considerable higher rate. This might result in a re-association to a new BS. First, we introduce an advanced UIL scheme (AdvUIL-load) whereby the utility function includes the BS loads to make sure that the network load is balanced among BSs. Furthermore, an improved UIL scheme (AdvUIL-load-backhaul) can be considered whereby the utility function is constructed based on the BS load data as well as the BS backhaul capacity.

In Adv-UIL-load scheme, for a UE $u$, originally located at $x_{u}^{0}$, the utility function is defined as

$$
U_{u}^{x}=\frac{W \eta_{x} p_{x}}{N_{x}}
$$

where $N_{x}$ is the number of UEs associated with the BS serving the point $x, W$ is the total available bandwidth, $\eta_{x}$ is the spectral efficiency at point $x$, and $p_{x}$ is the probability of the user $u$ moving to point $x$. This scheme takes into account the BS load, by incorporating $W / N_{x}$ into the utility formula, as well as the channel quality (i.e., the spectral efficiency). The location $x_{u}^{*}$, providing the highest utility, is suggested to the UE $u$ to move to.

In the Adv-UIL-load-backhaul scheme, the utility function for a UE $u$, originally 

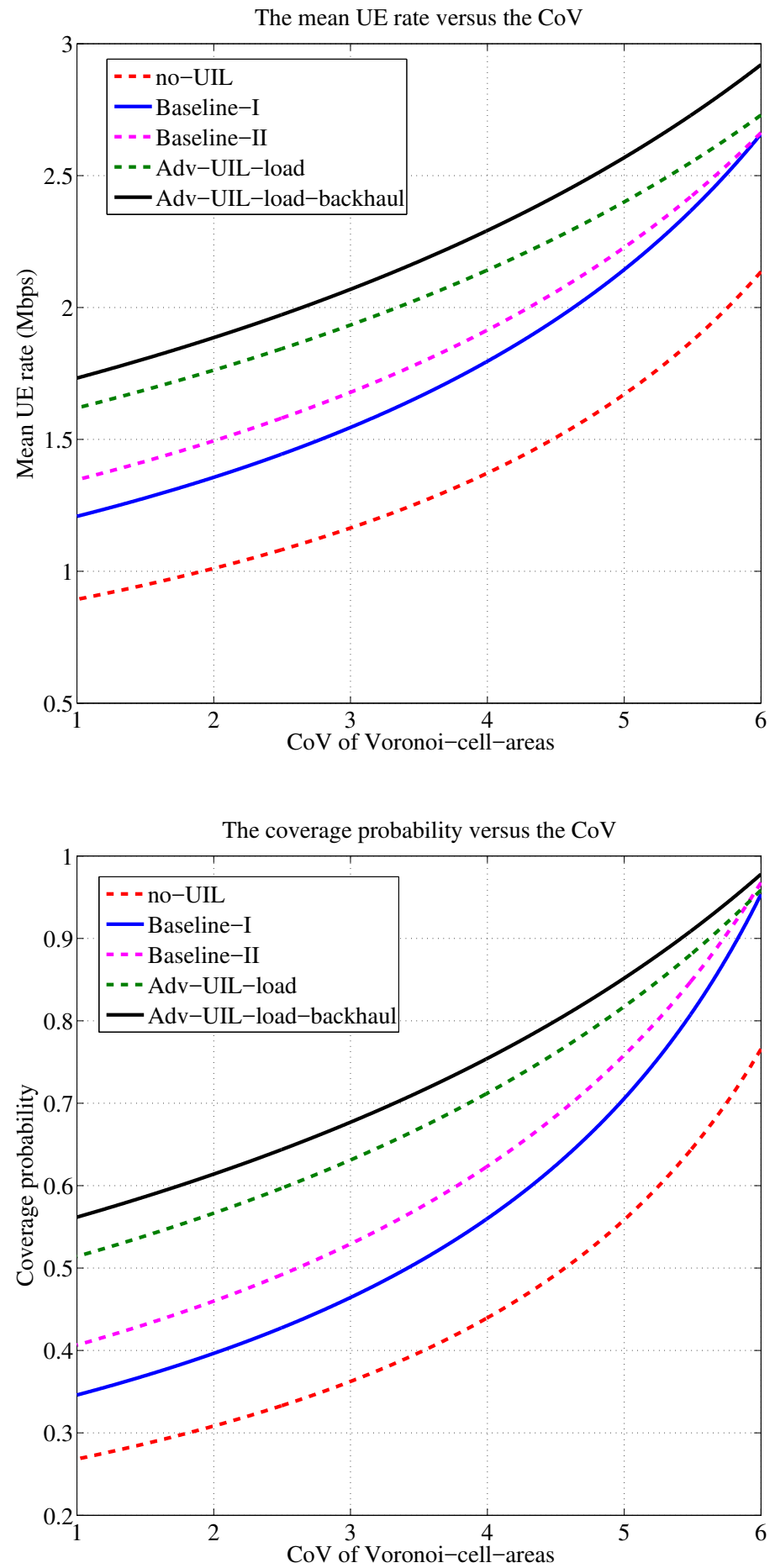

Figure 26: The mean user rate averaged over the entire network (left) and the coverage probability with minimum rate of $1 \mathrm{Mbps}$ (right) are shown in this figure (y-axis). The $\mathrm{x}$-axis shows the $\mathrm{CoV}$ values. Nonlinear least-squares fitting is used to show the curve trends. 
located at $x_{u}^{0}$, is defined as

$$
U_{u}^{x}=\min \left(1, \frac{C_{a_{x}}}{L_{a_{x}}}\right) \times \frac{W \eta_{x} p_{x}}{N_{x}}
$$

where $a_{x}$ is the BS associated with the point $x, C_{a_{x}}$ is the backhaul capacity of $a_{x}$, and $L_{a_{x}}$ is the aggregate load offered on $a_{x}$. In a network with finite backhaul capacity, for every point in the network, one of the following cases might happen:

1. The backhaul capacity is more than the wireless access link capacity. In this case, the backhaul capacity is not a bottleneck and every user receives the exact rate that is offered by the wireless access connection, i.e., $W \eta_{x} p_{x} / N_{x}$.

2. The backhaul capacity is less than the wireless access capacity. In this case, the backhaul capacity limits the user rates and every user receives just a certain portion of the available access rate. In other words, the wireless access rate for each point is decreased by a factor of $C_{a_{x}} / L_{a_{x}}$.

In the future HetHetNets where the backhaul limitation is predicted to be a real issue, the proposed UIL schemes can have considerable impact. In Section 4.5, we present the simulation results.

\subsection{Simulation Results}

Based on the HetHetNet modeling proposed in Section 4.2 and the proposed UIL schemes in Section 4.4, in this section, we present the simulation parameters and the experimental results.

We used a MATLAB-based simulation environment. UEs with mean overall density of $\lambda_{u}=1000 / \mathrm{km}^{2}$ were dropped in a $1 \mathrm{~km} \times 1 \mathrm{~km}$ network area. A 3-tier HetNet is simulated consisting of macro-BSs with mean density of $\lambda_{1}=5 / \mathrm{km}^{2}$, pico-BSs with mean density of $\lambda_{2}=25 / \mathrm{km}^{2}$, and femto-BSs with mean density of $\lambda_{3}=25 / \mathrm{km}^{2}$. The macro-BSs, pico-BSs, and femto-BSs are assumed to be connected to the backhaul network with capacities of $C_{1}=1 \mathrm{Gbps}, C_{2}=100 \mathrm{Mbps}$, and $C_{3}=10 \mathrm{Mbps}$, respectively, all with omni-directional antennas. We repeated the experiment for 1000 drops and gathered the results for each drop. The UEs are presumed to have fullbuffer traffic model in the time domain. As suggested by 3rd Generation Partnership Project (3GPP) technical specification group for Evolved Universal Terrestrial Radio 
Access (E-UTRA), the transmit power for macro, pico, and femto BSs are set to be $P_{1}=46 \mathrm{dBm}, P_{2}=30 \mathrm{dBm}$, and $P_{3}=23 \mathrm{dBm}$, respectively. Table 5 summarizes the simulation parameters used in this chapter.

The network mean user rates versus the $\mathrm{CoV}$ of Voronoi-cell-areas is illustrated in Fig. 26. As shown in Fig. 26, with decrease in $\mathrm{CoV}$ (with increase in $p$, i.e., the ratio of BS-independent UEs), the network mean user rate is decreased as expected. This is because the number of users which are near BSs and have high quality channel status is decreased. Moreover, the new proposed UIL schemes, namely Adv-UILload and Adv-UIL-load-backhaul, show superior performance compared to the basic schemes. This is due to the fact that in the advanced proposed schemes, if the backhaul connection of a BS does not have enough capacity to support new traffic, even if the access connection is in good quality, the UE is not guided to this BS. However, in the basic UIL schemes, the backhaul capacity is not taken into account.

The next metric which is investigated is the coverage probability. Figure 26 illustrates the coverage probabilities versus the CoV of Voronoi-cell-areas. The minimum rate for coverage can be a simulation parameter. In this chapter, we set the minimum rate threshold to be $1 \mathrm{Mbps}$.

It can be seen again that the coverage probability is increased by the UIL schemes and the new proposed schemes improve the coverage probability compared to the basic UIL schemes. For highly uncorrelated traffic to the base stations, the coverage probability is enhanced from less than $30 \%$ to about $60 \%$ (more than doubled). However, note that the realistic distribution of traffic is not represented by this uniform case $(p=1)$.

\subsection{Conclusion}

We proposed an adjustable spatial traffic model for HetHetNets with limited backhaul connection capacity. With the novel network model, we introduced improved UIL schemes whereby the BS loads and the BS backhaul connections are taken into account. The simulation results confirm that the new UIL scheme improves such important network performance metrics as the mean user rate and the coverage probabilities in the future $5 \mathrm{G}$ networks. 


\section{Chapter 5}

\section{VN Admission Control in HetHetNets}

\section{$5.1 \quad$ Introduction}

In future software-defined networks (SDNs), wireless access service is envisioned to be requested not only for individual users but also for groups of users. The user groups are assigned with virtual slices of the network called virtual networks (VNs). VN customers request service from infrastructure providers who operate substrate networks (SNs). Examples of SNs include future $5 \mathrm{G} / 5 \mathrm{G}+$ cellular networks and future Wi-Fi networks, and examples of VN customers include taxi operators, Police, bus operators, post companies, second-level service providers, and many other private and public organizations. Figure 27 shows an example of network structure in a SDN.

Upon arrival of VN service requests, which might have heterogeneous traffic profiles and different quality-of-experience (QoE) requirements, a central network admission control entity, which can be considered as a network function in the network operating system (NOS), must decide to whether admit or reject the customer service request. Admission control is performed based on a large number of parameters including the customer traffic profile (user equipment (UE) locations), available bandwidth at base stations $(\mathrm{BSs})^{1}$, and customer QoE requirements.

The virtual network admission control in a wireless network is different from (and more complicated than) the regular single-session admission control. In the singlesession admission control, decision is made based on a (deterministic) snapshot of

\footnotetext{
${ }^{1}$ Since the network discussed in this chapter is general and there is no assumption on network technology, the general term BS is used to refer to any serving point. Therefore, BS might refer to access points (APs) in Wi-Fi, evolved Node BSs (eNodeBs) in LTE, or serving nodes in other technologies.
} 


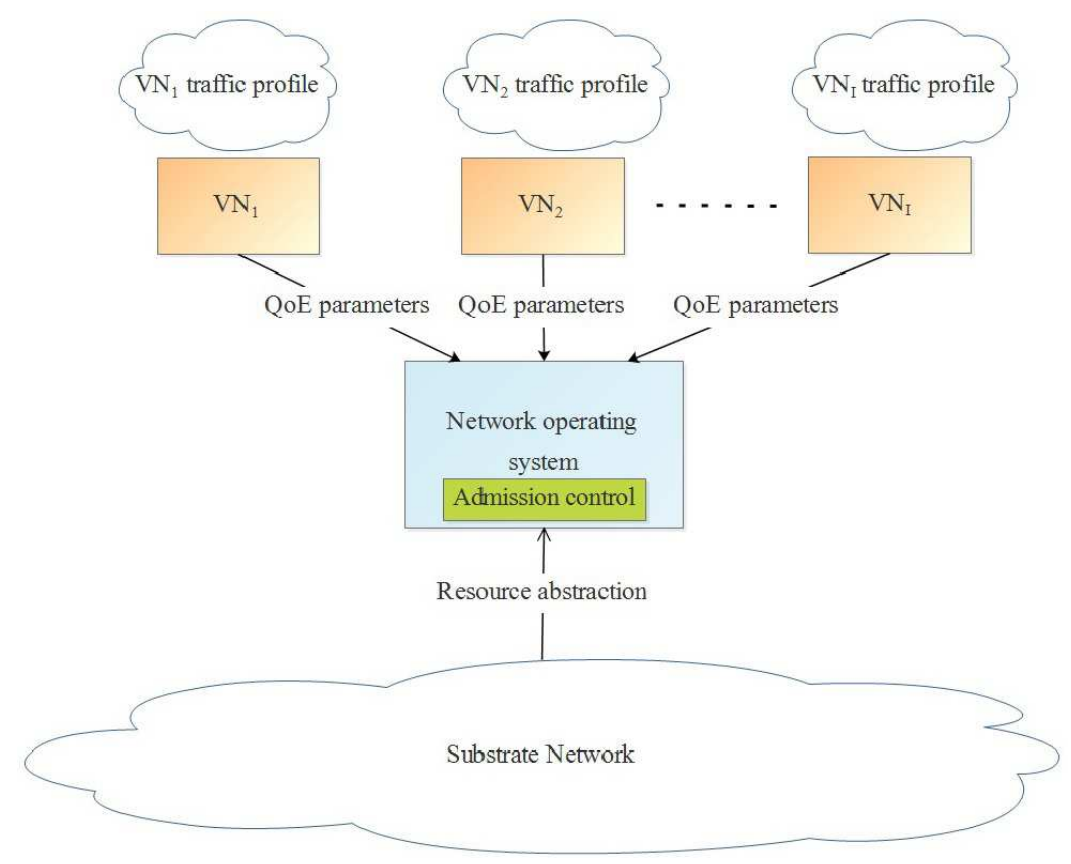

Figure 27: Network structure: admission control, as a function in NOS, decides on admission or rejection of $\mathrm{VN}$ requests based on the customer traffic profiles, required QoE, and resource availability in SNs.

already existing UE locations and the new UE location; while in the VN admission control, the UE locations and traffic demand distribution information are provided statistically by the VN customers. For instance, VN customers might indicate the expected distribution of traffic in various areas of the network. Therefore, the VN requests must be admitted if their QoE can be satisfied statistically. After (longterm) admission of a VN, (short-term) single-sessions of the VN are admitted only if they follow the restrictions and limitations of the $\mathrm{VN}$ admission contract. Illustration of the VN admission control concept and its relation to the regular single-session admission control is given in Figure 28.

In future $5 \mathrm{G} / 5 \mathrm{G}+$ networks, an envisioned characteristic of the networks is heterogeneity. It is expected that the network infrastructure will be heterogeneous in $5 \mathrm{G} / 5 \mathrm{G}+$ networks including different types of access points and base stations such as macro-BSs, pico-BSs, and femto-BSs. On the other hand, due to the emergence of various applications with different rate requirements, the traffic distribution is becoming highly heterogeneous (non-uniform) ${ }^{1}$ both in the time domain and the space

\footnotetext{
${ }^{1}$ In this chapter, we use the terms "heterogeneous" and "non-uniform" interchangeably. Similarly,
} 


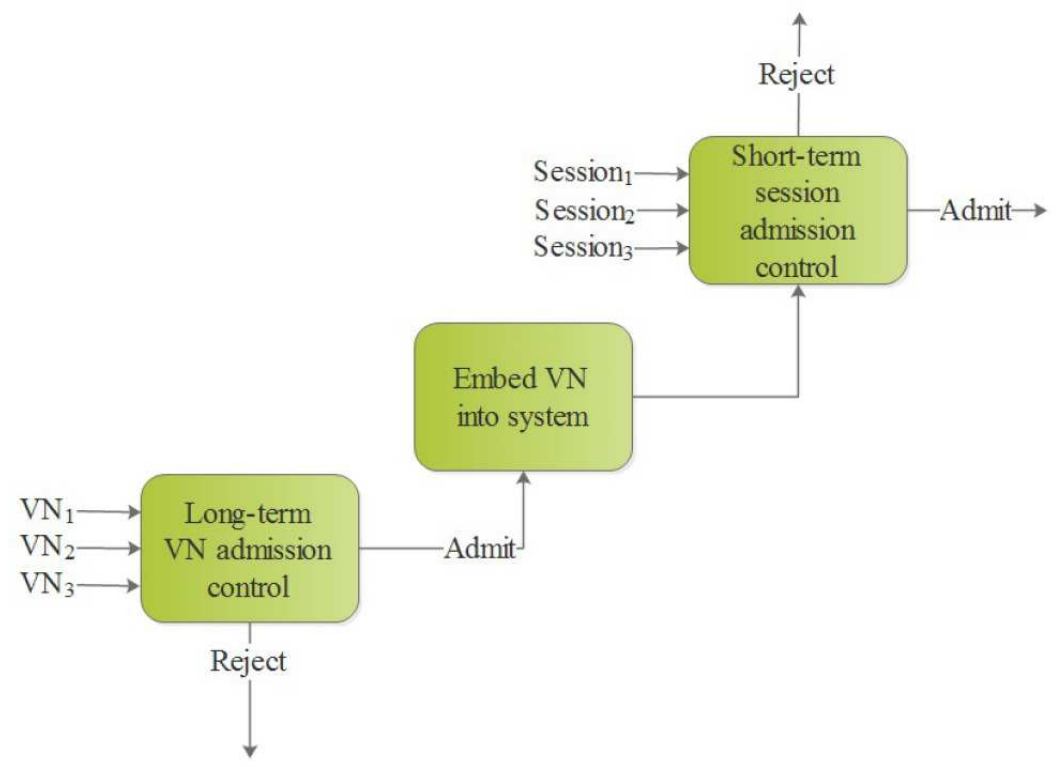

Figure 28: Admission control is performed in two levels: in the long-term level, VNs are admitted and embedded into the system statistically based on resource availabilities, and in the short-term level, single-sessions are admitted based on VN customer contracts.

domain. Therefore, a desirable admission control mechanism must be able to work in heterogeneous wireless networks with heterogeneous traffic distributions (HetHetNets).

In this chapter, a novel mechanism for admission control of VN requests is proposed which is also applicable to HetHetNets. The VN admission control construct is formulated as a convex problem which is tractable and efficient to solve.

The main contributions of this chapter are summarized as follows:

- We propose a wireless VN admission control mechanism which incorporates feedback information to the customer to update its traffic profile according to the real traffic measurements conducted by the VN service provider. This results in more accurate traffic specification and better QoE. The proposed methodology is applicable to any traffic profile including heterogeneous scenarios and HetHetNets.

- We formulate VN admission control as a convex optimization problem which is computationally tractable and efficient. We also present a number of extensions we use the terms "homogeneous" and "uniform" interchangeably. 
which incorporate network cost minimization and network backhaul limitations in the problem.

- Our proposed VN admission control method allows general multiple association between UEs and BSs. The simulation results verify that a higher number of $\mathrm{VN}$ requests can be admitted to the network using the proposed technique compared to the existing methods.

\subsection{System Model and Problem Definition}

In this section, we describe the system model and parameters (Section 5.2.1), and define the wireless VN admission control problem in specific terms (Section 5.2.2).

\subsubsection{System Model}

We consider a software-defined radio access network (SD-RAN) with limited backhaul capacities and general access parts. Target systems could be, but are not limited to, WiMAX2, Wi-Fi, 4G LTE, 5G/5G+ HetNets or a combination of them. We focus on downlink in this chapter but our method is also applicable to uplink. In particular, in this chapter we focus on admission or rejection of VNs according to the downlink requirements. However, the uplink requirements, or a combination of downlink and uplink requirements, can also be considered.

We consider a geographical region $L \subset \mathbb{R}^{2}$ (i.e., two dimensional plane) as the network layout that is served by a set of BSs $B$. Each BS is characterized by its transmit power, backhaul capacity $H_{k}, k \in K=\{1, \ldots,|B|\}$, and its available bandwidth $W_{k}$, as shown in Figure 29. In this chapter, we always use $k$ as the index for BSs.

Let $l \in L$ denote a location on the network layout. We assume that demand arrivals for the $i$ th $\mathrm{VN}, v_{i} \in V$, at location $l$ and at time $t, d_{i l}^{t}$, are random variables that follow arbitrary distributions with mean $\lambda_{i l}^{t}$, where $V$ is the set of VNs. This provides flexibility for inhomogeneous, i.e., heterogeneous or non-uniform, traffic characterization. We always use $i$ for indexing VNs. Detailed investigation of heterogeneous traffic modeling is performed in [26-28]. To avoid notation complexity, we drop the time index $t$ in this chapter. It can be shown that our results can be easily extended to span time-varying traffic statistics by profiling traffic for specific times of day, week, or year. Moreover, to avoid unnecessary technical difficulties, in this 


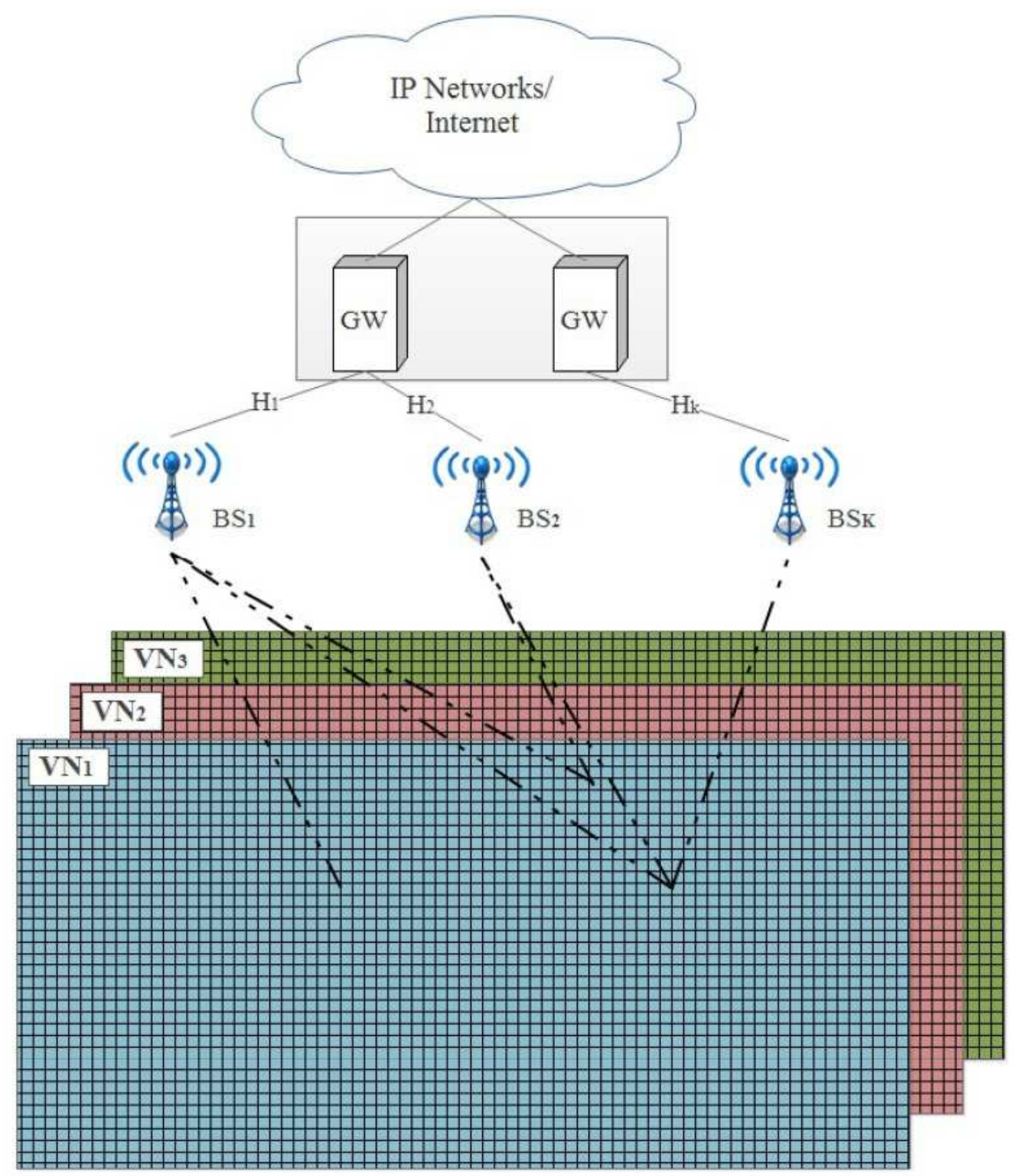

Figure 29: System model: VNs with heterogeneous traffic profiles to be served by a general SN with backhaul capacity limits. Every bin can be associated to one, two, or multiple BSs. 
chapter, we assume that the region $L$ is divided into a set of small (e.g., $5 \mathrm{~m} \times 5 \mathrm{~m}$ ) bins (areas) $a \in A$, and the demand for each bin is defined as

$$
d_{i j}=\int_{l \in a_{j}} d_{i l}, j \in J, i \in I,
$$

where $j$ is used for indexing bins, $J=\{1, \ldots,|A|\}$ is the set of bin indices, and $I=\{1, \ldots,|V|\}$ is the set of $\mathrm{VN}$ indices.

In this chapter, we assume that the BSs transmit with constant (not necessarily equal) power; we leave power control extension as a future work. The signal-tointerference-plus-noise ratio (SINR) for the center of bin $a_{j} \in A$ if it is connected to BS $b_{k} \in B$ is defined as

$$
\gamma_{j k}=\frac{P_{j k}}{P_{N}+\sum_{j^{\prime} \in J \backslash j} P_{j^{\prime} k}},
$$

where $P_{j k}$ is the effective received power from BS $b_{k}, k \in K$ at bin $a_{j}$, and $P_{N}$ is the noise power.

The received power can be calculated based on path-loss exponent and channel models as well as the other environmental parameters. In practical cases, it also can be collected from field measurements. Spectral efficiency (SE) can be any arbitrary function of SINR in this method. In our simulations, we assume that it follows the Shannon's formula as follows:

$$
\eta_{j k}=\log _{2}\left(1+\gamma_{j k}\right)
$$

For the sake of a better readability and an easier understanding, a list of parameters used in this chapter with associated symbols are summarized in Table 6.

\subsubsection{Problem Definition}

In this chapter, we assume that QoE expectations are specified by each customer and each $\mathrm{VN}, v_{i} \in V$, by three main parameters:

1. the required traffic demand which is specified by the traffic demand matrix $d$, or more specifically, by the distribution parameters of traffic in each bin,

2. the required maximum outage $O_{i}$ (or equivalently, the required minimum coverage probability $C_{i}$ ), 
Table 6: List of Symbols

\begin{tabular}{|c|c|}
\hline Symbol & Definition \\
\hline$L$ & Network region \\
\hline$B$ & Set of BSs \\
\hline$b$ & A typical BS \\
\hline$l$ & A typical location \\
\hline$d$ & Traffic demand matrix \\
\hline$\lambda$ & Traffic demand mean matrix \\
\hline$t$ & Time index \\
\hline$A$ & Set of bins \\
\hline$a$ & A typical bin \\
\hline$V$ & Set of VNs \\
\hline$P$ & Received power matrix \\
\hline$P_{N}$ & Noise power \\
\hline$\gamma$ & SINR matrix \\
\hline$\eta$ & SE matrix \\
\hline$U$ & BS maximum utilization array \\
\hline$O$ & Outage requirement array \\
\hline$C$ & Coverage requirement array \\
\hline$D$ & Delay requirement array \\
\hline$H$ & BS backhaul capacity array \\
\hline$W$ & BS available bandwidth array \\
\hline$x$ & Rate association matrix \\
\hline M & BS loads array \\
\hline$r$ & Total received rate matrix \\
\hline$\Delta$ & Average of experienced delay matrix \\
\hline$\Theta$ & Experienced delay matrix \\
\hline$\xi$ & Experienced coverage matrix \\
\hline$\chi$ & Network cost \\
\hline
\end{tabular}




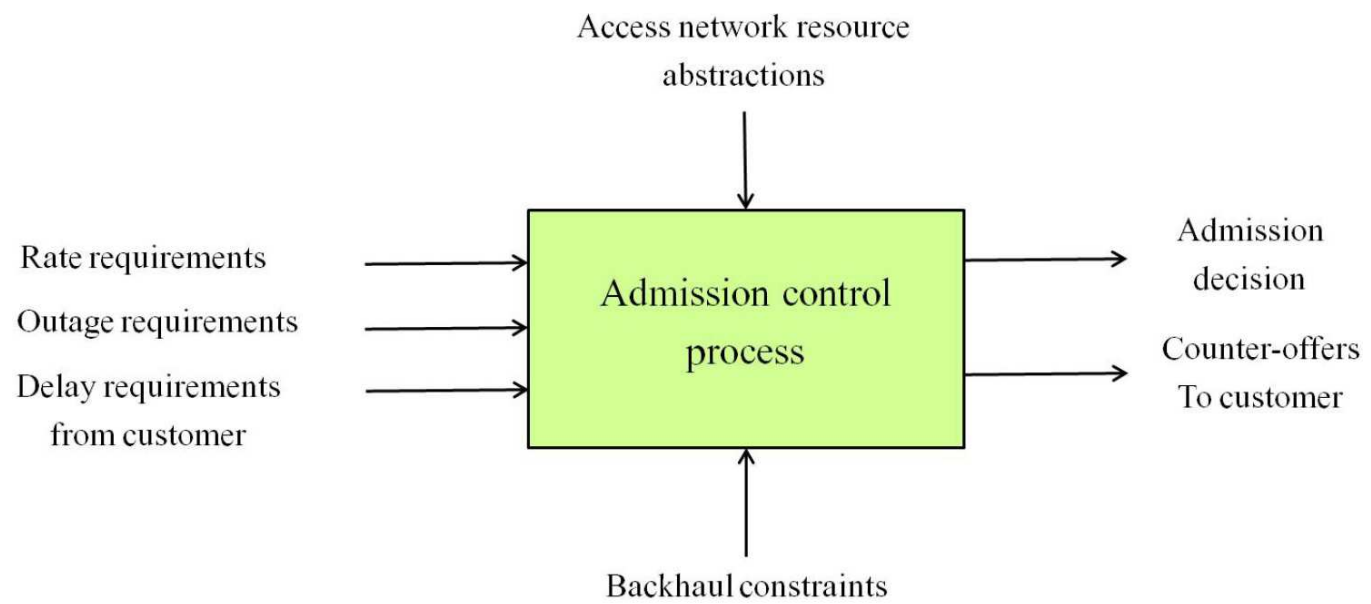

Figure 30: Specific input parameters and outputs of the admission control process are illustrated.

3. and the required maximum delay $D_{i}$, or the delay thresholds with associated probabilities.

In the heterogeneous wireless network system described in Section 5.2.1, VN requests arrive with specific traffic profiles and QoE requirements. Upon the arrival of each new VN request, the VN admission control problem must be executed and solved to decide whether or not the newly arrived VN request can be admitted and incorporated in the system, with all of its QoE requirements satisfied, and without affecting the already existing VNs' services.

Figure 30 shows the input parameters and the side information which is entered in the admission control module along with the possible outputs driven from solving the admission control problem.

\subsection{Admission Control for Virtual Networks}

In this section, the admission control process and method for virtual networks is described. Section 5.3.1 describes the broad view of the VN admission control process. The multiple association optimization method for $\mathrm{VN}$ admission control is explained in Section 5.3.2, and proved to be convex in Section 5.3.3. Two extensions are added to the optimization framework in Section 5.3.4. 


\subsubsection{Admission Control Process}

The broad view of the proposed VN admission control process is illustrated in detail in Figure 31. Figure 31 depicts a comprehensive procedure for admission control of VNs which includes the interface to the customers and network operation feedback. The various steps of the process are explained from upper-left corner as follows:

- The VN customers provide their QoE expectations including the demand statistics, outage requirements, and delay requirements.

- The demand statistics might be provided by the customer in a resolution different than the network. Therefore, they must be converted to the network bin resolution. The customer may specify the overall mean rate only, he can specify some hotspots, or he can specify very detailed demand information.

- The VN admission control problem is executed and solved using convex optimization method explained later in Section 5.3.2. This part is the main problem solving element of the $\mathrm{VN}$ admission procedure.

- If a $\mathrm{VN}$ is admitted to the system, after embedding the VN into system, the scheduling is done opportunistically, even though the admission was done statistically. This means that a better QoE can be achieved in comparison to what admission control is based on. On the other hand, the network operator might be aware of some inefficiency which cannot be quantified in the admission control problem. Therefore, the network operator may decide to do an aggressive or conservative admission.

- If the final decision is to reject the new VN request, there are some alternative actions including: deployment of new access points (e.g., femto or pico at hotspots), negotiating the QoE parameters with customer, borrowing some resources from the other infrastructure providers, and guiding customers to move to better locations if possible (e.g., user-in-the-loop [9]).

- If the final decision is to admit the new VN request, then the new VN is embedded into the system (resources are reserved and setup is done). In the operation phase, scheduling can be performed with power control and inter-cell interference coordination (ICIC) [81]. 
- A very important part of the VN admission control process is monitoring. Monitoring the network includes measurement of VN traffic and network utilization. Monitoring provides three main advantages:

1. Network resource utilization can be measured. This information can be fed back to the admission control function. For instance, if the utilization is low, then an aggressive admission can be performed and if the utilization is very high, then admission must be conducted conservatively. Presume that always less than $U_{k} \%$ of the resources in $\mathrm{BS} b_{k}$ are used during the network operation. In this case, the effective bandwidth for this BS that can be used for future admission control can be $W_{k} / U_{k}$.

2. Traffic demands for VNs must be measured. This assures that the customers are following the contracts. For instance, the mean and the standard deviation of the traffic for each $\mathrm{VN}$ at each bin can be calculated to make sure that it follows the promised statistics.

3. The actual measured traffic statistics can be fed back to customers (for a particular fee) to correct their initial estimations. Many VN customers don't have the measurement facilities and can benefit from this information and correct their traffic profiles.

The explained process is a comprehensive reference procedure that might be implemented partially or fully by a network operator to consider different aspects of VN admission control. In this chapter we focus on the main admission problem which is cast as a convex optimization problem in Section 5.3.2.

\subsubsection{Admission Control Method}

While the simplest (and mostly used) UE-BS association is based on maximum received power and maximum SINR (max-SINR), technology advances in future wireless networks allow for general multiple association in which a UE (or a bin) can be associated to multiple BSs, or to a BS with low SINR but high available bandwidth.

It is shown in the recent literature [82-84] that general association can result in significant improvement in network key parameter indicators, specially in future HetNets where small cells have limited coverage areas hence lower load compared to 


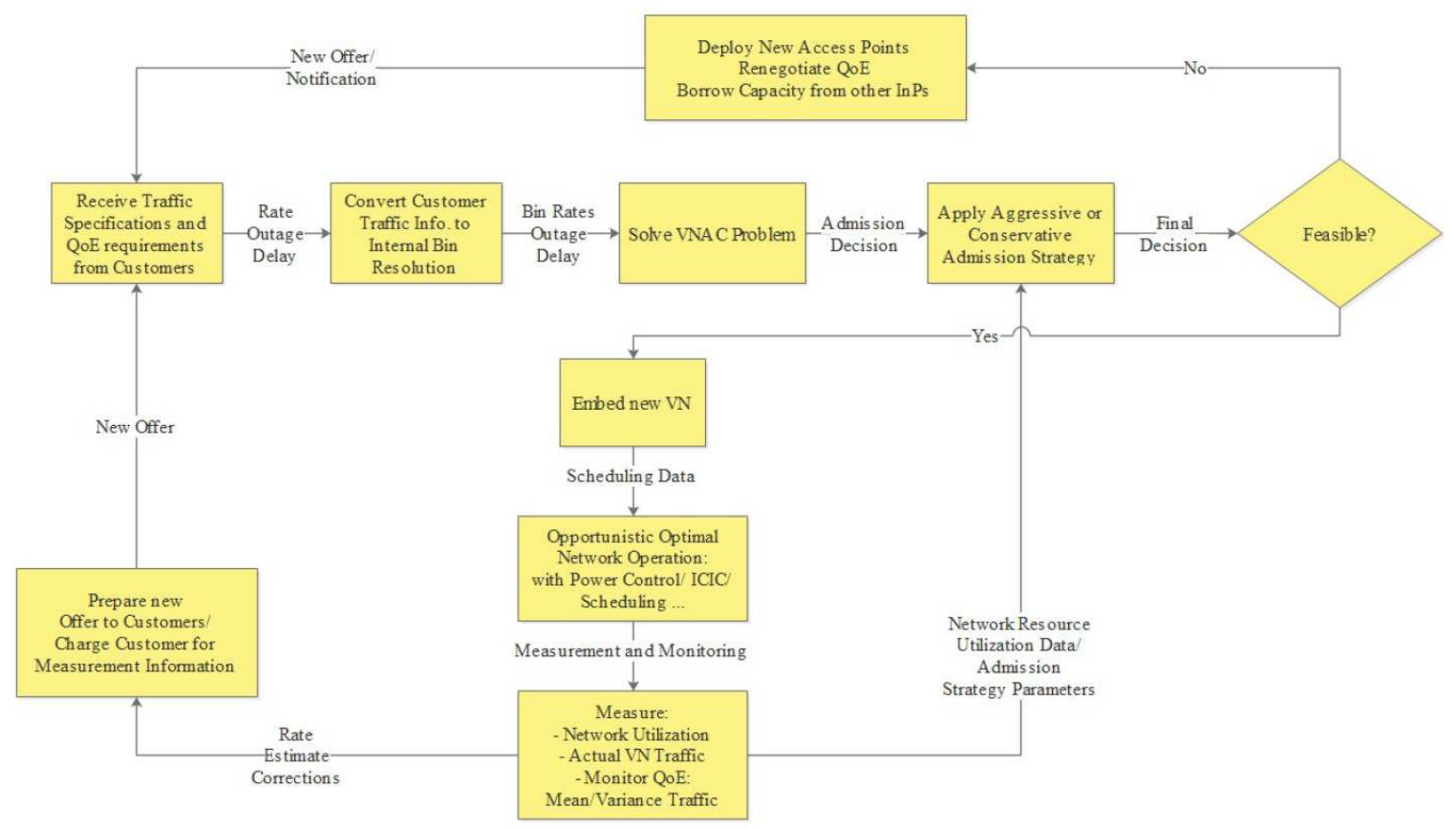

Figure 31: VN admission control process: network-wide traffic measurement provides feedback information for the customers, traffic monitoring for QoE assurance, and possibility for an aggressive admission. 


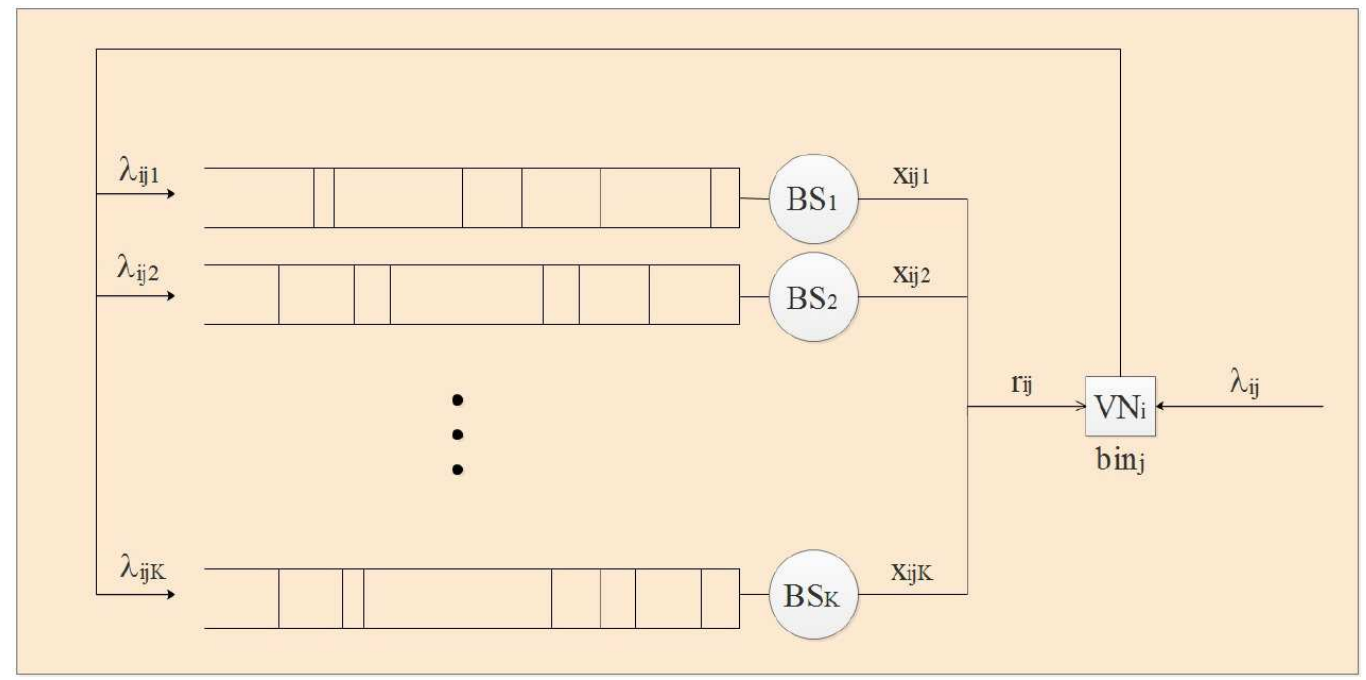

Figure 32: The downlink queues in BSs: every bin is served by multiple BSs with downlink queues which have different arrival rates and service rates which can be modeled by separate parallel $\mathrm{M} / \mathrm{M} / 1$ queues.

macro cells. In this section, a VN admission control method with general association is proposed.

First, note that VN admission control problem is a feasibility problem in which we are not trying to improve an objective function as the ultimate goal is to determine if some admission control constraints can be satisfied or not. Let $x_{i j k}$ denote the amount of rate received from $\mathrm{BS} b_{k} \in B$ at bin $a_{j} \in A$ by $\mathrm{VN} v_{i} \in V$. The objective of the optimization is to find a solution $x^{*}$ that satisfies optimization constraints.

The first constraint is that the sum of the resources allocated by each BS to all bins cannot exceed its available resources:

$$
\mathrm{C} 1: \quad M_{k}=\sum_{i \in I} \sum_{j \in J} \frac{x_{i j k}}{\eta_{j k}} \leqslant W_{k}, \forall k \in K
$$

where $M_{k}$ is the amount of used resources from BS $k, W_{k}$ is the available resources, and $\eta_{j k}$ is the effective spectral efficiency experienced from BS $k$ at bin $j$.

Assuming that session inter-arrival time in the time domain is exponentially distributed and is independent at all bins for all VNs, and the session sizes are distributed independently and exponentially, the downlink queues in BSs can be modeled by parallel M/M/1 queues as illustrated in Figure 32.

We know that the total arrival rate for $\mathrm{VN} v_{i} \in V$ at bin $a_{j} \in A$ is $\lambda_{i j}$. Now 
assume that the arrived session requests are sent to BS $b_{k} \in B$ with probability $p_{i j k}$. Then, the effective arrival rate for this $\mathrm{BS}$ is $\lambda_{i j k}=p_{i j k} \lambda_{i j}$ where

$$
\sum_{k \in K} p_{i j k}=1, \quad \forall i \in I, \forall j \in J
$$

Therefore, the second constraint is as follows:

$$
\mathrm{C} 2: \quad \sum_{k \in K} \lambda_{i j k}=\lambda_{i j}, \quad \forall i \in I, \forall j \in J
$$

Based on Burke's theorem on parallel queues [85], the average service delay for each bin $a_{j} \in A$ for each $\mathrm{VN} v_{i} \in V$ from BS $b_{k} \in B$ is defined as

$$
\Delta_{i j k}=\frac{1}{x_{i j k}-\lambda_{i j k}}
$$

The third constraint is that each VN at each bin cannot experience a delay higher than its required maximum delay (note that delay requirement is defined only for time sensitive services). The maximum experienced delay by each $\mathrm{VN}$ at each bin is the maximum experienced delay in all parallel queues. Therefore,

$$
\max _{k \in K} \Delta_{i j k} \leqslant D_{i}, \forall i \in I, \forall j \in J
$$

Delay requirement can also be defined by customers for every single bin. In that case, $D_{i j}$, the delay requirement matrix will replace $D_{i}$, the delay requirement array. However, this is not a common case and is not considered in this thesis.

In (53), the maximum delay for all parallel queues must be less than or equal to the delay threshold. This means that all delays for all parallel queues must be less than or equal to the delay threshold (third constraint):

$$
\text { C3: } \quad \Delta_{i j k} \leqslant D_{i}, \forall i \in I, \forall j \in J, \forall k \in K \text {. }
$$

The total delay experienced by UEs is the sum of all delays from the moment that the service request is submitted to the wireless system until the moment when the UE receives the service. For instance, backhaul transmission might add considerable delay in service. Processing delay (in BS as well as in UE) and propagation delay also must be added to the total delay. In this chapter, we only considered the queuing 
and wireless service delay for downlink part, assuming that other delays sum up to zero.

For all the queues in the BSs to be stable, it is also necessary that all arrival rates in all queues be less than service rates:

$$
\text { C4: } \quad \lambda_{i j k} \leqslant x_{i j k}, \forall i \in I, \forall j \in J, \forall k \in K \text {. }
$$

Also note that in (54) we are limiting the maximum of the average experienced delays $\Delta_{i j k}$ to be less than tolerance threshold $D_{i}$. However, this can be extended to any percentile of delays. To be more specific, assume that we want the experienced delays $\Theta_{i j k}$ to be less than a threshold $D_{i}$ with a probability of $q$ :

$$
\operatorname{Pr}\left\{\Theta_{i j k} \leqslant D_{i}\right\} \geqslant q, \forall i \in I, \forall j \in J, \forall k \in K
$$

This means that we want the cumulative distribution function (CDF) of delays to be greater than $q$ at $D_{i}$ :

$$
F_{\Theta_{i j k}}\left(D_{i}\right) \geqslant q, \forall i \in I, \forall j \in J, \forall k \in K
$$

From M/M/1 queue analysis, it is known that the delay has an exponential distribution with parameter $x_{i j k}-\lambda_{i j k}$ [86]. Therefore, (57) can be written as

$$
1-\exp \left(-D_{i}\left(x_{i j k}-\lambda_{i j k}\right)\right) \geqslant q, \forall i \in I, \forall j \in J, \forall k \in K,
$$

which can be in turn simplified to

$$
x_{i j k}-\lambda_{i j k} \geqslant \frac{-\log (1-q)}{D_{i}}, \forall i \in I, \forall j \in J, \forall k \in K
$$

Finally, the last constraint is that coverage probability for all customers must be higher than the minimum required coverage. The coverage probability for a VN $v_{i} \in V$ for one bin $a_{j} \in A$ is equal to the probability that the demand for this $\mathrm{VN}$ in this bin is less than its received rate from all BSs:

$$
\xi_{i j}=\operatorname{Pr}\left\{d_{i j} \leqslant r_{i j}\right\},
$$


where $r_{i j}$ is the total rate received by $\operatorname{VN} v_{i} \in V$ at bin $a_{j} \in A$ :

$$
\text { C5: } \quad r_{i j}=\sum_{k \in K} x_{i j k}, \forall i \in I, \forall j \in J .
$$

To calculate the coverage probability for a VN over the entire network, one way is to take the normal average. However, since different bins have different traffic demands, a better measure is a weighted average:

$$
\xi_{i}=\frac{1}{\lambda_{i}}\left(\sum_{j \in J} \lambda_{i j} \cdot \xi_{i j}\right),
$$

where $\lambda_{i}=\sum_{j \in J} \lambda_{i j}$ is the total demand for $\mathrm{VN} v_{i} \in V$. Therefore, the last constraint is

$$
\text { C6: } \quad \xi_{i} \geqslant C_{i}, \forall i \in I
$$

In summary, the VN admission control problem can be cast as the following problem:

$$
\text { find }_{x, r, \lambda} x \text { subject to: C1-C6. }
$$

\subsubsection{Convexity of the Problem}

A mathematical optimization problem can be solved efficiently and methodically if it can be cast as a convex problem [87]. A problem is convex if the objective function and the constraints are convex functions. In this section, we show that the problem (64) is convex and can be solved efficiently with well-known convex optimization tools and optimization methods such as interior point methods. Interior point methods are a certain class of algorithms that solve linear and nonlinear convex optimization problems [87].

In (64), the constraints C1-C5 are convex w.r.t. $x, \lambda$, and $r$. Therefore, the problem is convex if the last constraint (C6) is convex. First, note that C6 is convex if $\xi_{i}$ is a concave function of $r$. Also note that $\xi_{i}$ is a summation of $\xi_{i j}$. Therefore, it is concave if $\xi_{i j}$ is a concave function w.r.t. $r$. From (60) it is clear that $\xi_{i j}$ is the CDF of $d_{i j}$ at $r_{i j}$. Therefore, (64) is a convex optimization problem if $d$ has a concave CDF. It can be easily shown that the exponential distribution and uniform distribution have concave CDFs. The CDF of the exponential distribution is defined 
as

$$
F_{X}(x)=1-\exp (-\Lambda x)
$$

for parameter $\Lambda$, which has the following second derivative:

$$
\frac{d^{2} F}{d x^{2}}=-\Lambda^{2} \exp (-\Lambda x)
$$

which is always negative.

The CDF of uniform distribution is defined as

$$
F_{X}(x)=\frac{x-a}{b-a}
$$

for parameters $a$ and $b$, which is a linear function.

It can also be shown that Gaussian distribution has a concave CDF for $r \geqslant \lambda$. The CDF of the Gaussian distribution is defined as

$$
F_{X}(x)=\frac{1}{2}\left[1+\operatorname{erf}\left(\frac{x-\mu}{\sqrt{2} \sigma}\right)\right],
$$

for parameters $\mu$ and $\sigma$, where $\operatorname{erf}($.$) is the error function defined as$

$$
\operatorname{erf}(x)=E(x)=\frac{2}{\sqrt{\pi}} \int_{0}^{x} \exp \left(-t^{2}\right) d t
$$

which has the following second derivative:

$$
\frac{d^{2} E}{d x^{2}}=\frac{-4 x}{\sqrt{\pi}} \exp \left(-x^{2}\right)
$$

which is negative for positive $x$. Poisson distribution can be approximated by Gaussian distribution with very high accuracy for $\lambda>1000$ [88].

\subsubsection{Extensions}

Solving (64) returns a $x$ value that satisfies the constraints C1-C6. However, there is no guarantee that the result is the best result. For instance, there might be other $x$ values which satisfy the constraints and also result in lower energy consumption or network cost. 
The first proposed extension to problem (64) is to minimize the network cost while trying to find a solution which satisfies all constraints:

$$
\operatorname{minimize}_{x, r, \lambda} \chi \text { subject to: C1-C6, }
$$

where the network cost $\chi$ can be any convex function of the variables $x$. One common way of defining the network cost is an exponential function of BS load:

$$
\chi=\sum_{k \in K} e^{M_{k}}
$$

This definition results in a degree of load balancing among BSs. Another definition that results in min-max load balancing is the following:

$$
\chi=\max _{k \in K} M_{k} .
$$

In the formulation of $\mathrm{VN}$ admission control problem in (64), the backhaul capacities are not considered. In other words, there might be situations where the access part of a BS has enough capacity to serve high number of UEs while the backhaul capacity is limited. In particular, in the future $5 \mathrm{G} / 5 \mathrm{G}+$ HetNets, whereby femto BSs will be deployed by customers and operators will face deployment restrictions (or cost considerations) to lay fiber in many areas, it is not realistic to presume that backhaul links have unlimited capacities.

In a wireless network with ideal backhaul connections, the lack of capacity is mainly due to the access connection limitation, that is, the wireless access problem and low channel quality between UEs and BSs. However, in the envisioned 5G/5G+ networks with small cells deployed in residential and office buildings, the assumption of ideal backhaul is more optimistic than realistic, especially considering the fact that a main portion of the small cells (i.e., femto-cells) will be deployed by customers rather than service providers. Recently, there has been increasing interest in the literature in the study of limited backhaul capacities in future HetHetNets [76].

The next proposed extension for problem (64) is to take backhaul constraints,

$$
\text { C7: } \quad \sum_{i \in I} \sum_{j \in J} x_{i j k} \leqslant H_{k}, \forall k \in K
$$


into account and reformulate (64) as

$$
\text { minimize }_{x, r, \lambda} \chi \text { subject to: C1-C7. }
$$

\subsection{Simulation Results}

Considering the system explained in Section 5.2, in this section, the experimental results are presented that show the efficiency of the VN admission control methodology proposed in Section 5.3. The simulation parameters and setup are described in Section 5.4.1, and the simulation results are presented in Section 5.4.2.

\subsubsection{Simulation Setup and Parameters}

We use a MATLAB based simulation to verify our analytical results which are drawn in Section 5.3. The network is comprised of 19 macro-cells on the X-Y plane with three-sector antennas (57 sectors in total) and 57 pico-cells randomly and uniformly deployed in the plane with omni-directional antennas. The inter-site distance among macro-BSs is 150 meters. The antenna height is considered 32 meters for macro-BSs and 10 meters for pico-BSs.

The entire network is divided into $5 \mathrm{~m} \times 5 \mathrm{~m}$ bins and the spectral efficiency for the bin centers represents each bin. The path-loss exponent and the channel model is based on 3GPP Technical Report on further advancements for E-UTRA physical layer aspects (3GPP TR 36.814) [89]. The shadowing model is also from [89]. BS transmit power is $46 \mathrm{dBm}$ for macro-BSs and $30 \mathrm{dBm}$ for pico-BSs and fading is not considered (assumed to be averaged over time). The noise power is $P_{N}=-174$ $[\mathrm{dBm} / \mathrm{Hz}]$ and each BS has $10 \mathrm{MHz}$ bandwidth for downlink. Table 7 summarizes the simulation parameters used in this chapter.

\subsubsection{Simulation Results}

In the first experiment, we generate VNs with spatially uniform traffic, i.e., homogeneous Poisson point process (PPP). Each VN has mean traffic demand density of 0.2 Mbps at every bin. We increase the number of VNs and measure the maximum coverage probability (we assume that coverage requirement for all VNs is equal). Basically, 
Table 7: Simulation parameters for Chapter 5

\begin{tabular}{l|l}
\hline Parameter & Value \\
\hline Number of macro-BSs & 19 \\
Number of pico-BSs & 57 \\
Number of sectors for each macro-BS & 3 \\
Inter-site distance for macro-BSs & $150 \mathrm{~m}$ \\
Pico-cell deployment & uniform PPP \\
Macro-BS antenna height & $32 \mathrm{~m}$ \\
Pico-BS antenna height & $10 \mathrm{~m}$ \\
Bin size & $5 \mathrm{~m} \times 5 \mathrm{~m}$ \\
Noise power & $-174 \mathrm{dBm} / \mathrm{Hz}$ \\
Bandwidth for each BS & $10 \mathrm{MHz}$ \\
Macro-BS transmit power & $46 \mathrm{dBm}$ \\
Pico-BS transmit power & $30 \mathrm{dBm}$ \\
Shadowing & specified in $[89]$ \\
Macro-BS antenna gain & $14 \mathrm{~dB}$ \\
Pico-BS antenna gain & $5 \mathrm{~dB}$ \\
UE antenna gain & $0 \mathrm{~dB}$ \\
\hline
\end{tabular}




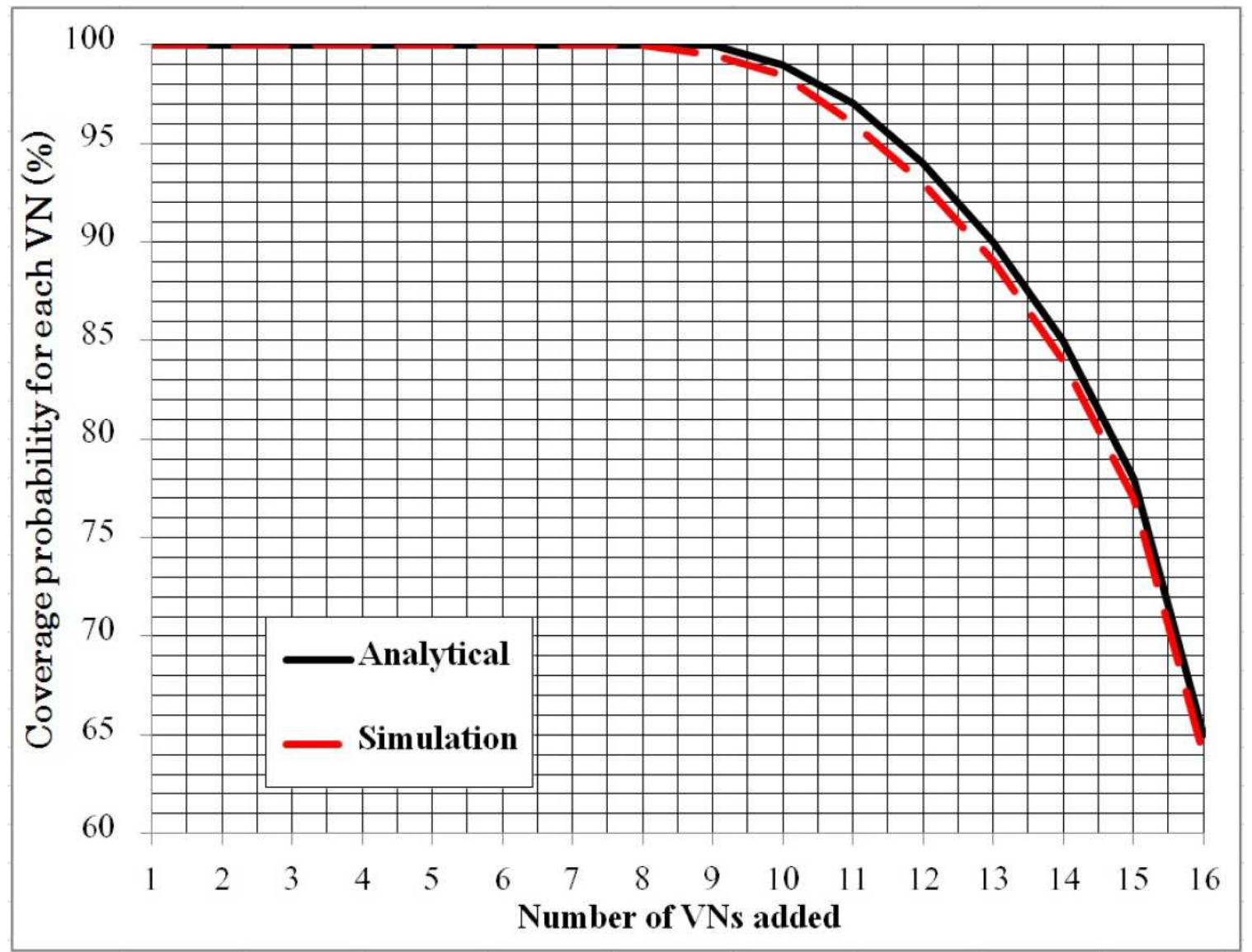

Figure 33: Analytical results versus simulation results: the small difference between the simulation and analytical results is in part due to the limited number of drops and shows the accuracy of the optimization results.

the Figure 33 illustrates the achievable coverages (customer satisfactions) versus the number of VNs. Obviously, with an increasing number of VNs, the coverage decreases.

We compare our analytical method (optimization) with simulation results obtained via simulation of 1000 drops of traffic demands. Here we assume that the rate given to a $\mathrm{VN}$ at a bin by each $\mathrm{BS}$ is fixed during network operation because the admission decision is made at the time of VN arrival. As presented in Figure 33, the simulation results verify the analytic method results.

In our $\mathrm{VN}$ admission control method, we use general association for bins, meaning that every bin can be served by multiple BSs. In Figure 34, we compare a VN 


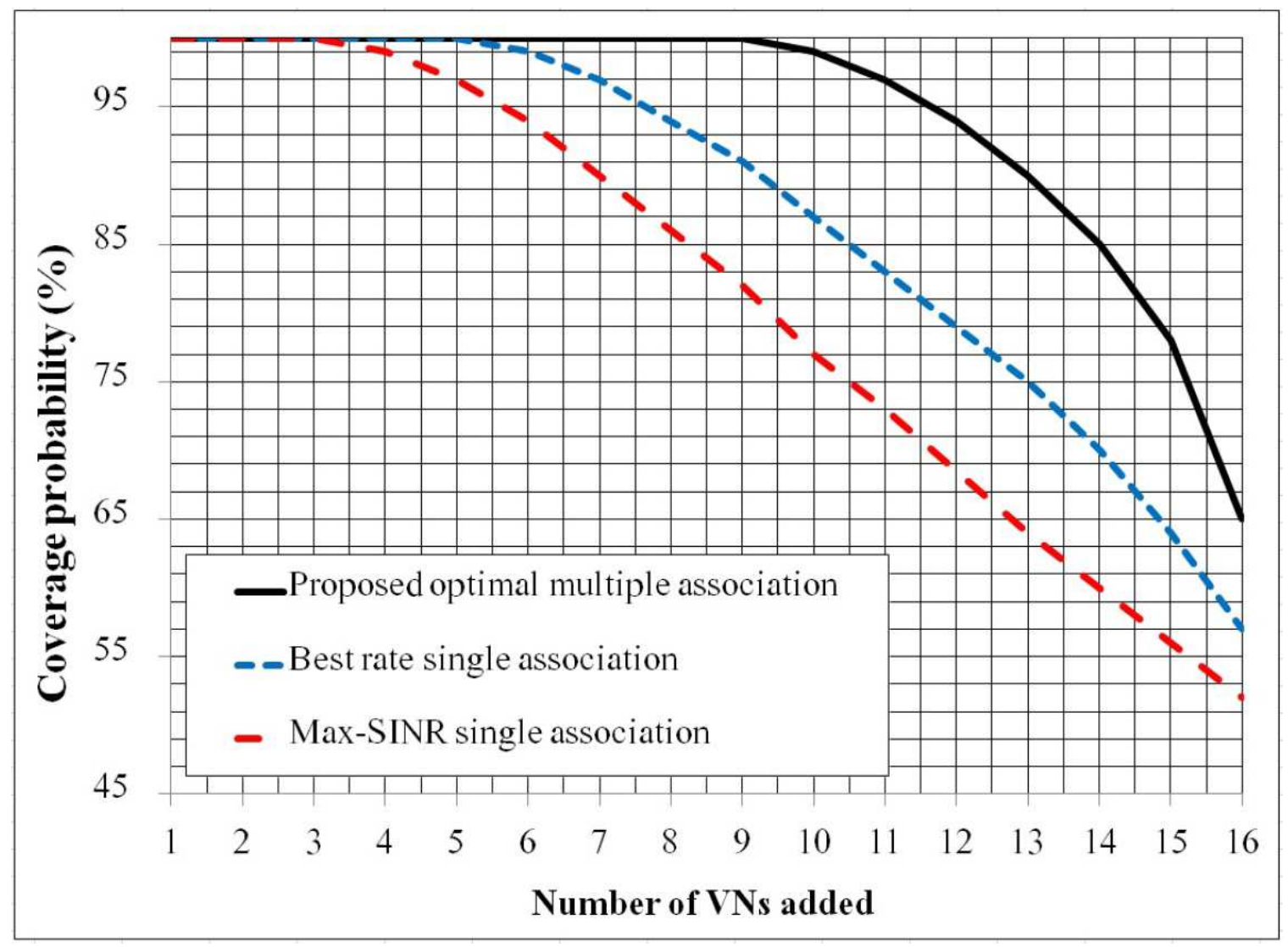

Figure 34: A VN admission control method with multiple association is compared to single association and max-SINR association methods. Multiple association leads to significant increase in network performance hence VN admission control results.

admission control method with multiple association to a VN admission control method without multiple association. The most popular alternative is max-SINR association in which each UE connects to the BS with most strong SINR. Another alternative (single association) is to run our optimization and find the rate values $x^{*}$; Then associate each $\mathrm{VN}$ at each bin to the BS with maximum rate, i.e.,

$$
m_{i j}=\arg \max _{k} x_{i j k} .
$$

Figure 34 shows that multiple association results in significant increase in network performance and subsequently number of admitted VNs with high QoE requirements.

Table 8 compares the number of VNs admitted with different QoE requirements 
Table 8: Multiple association versus max-SINR association

\begin{tabular}{|l|l|l|}
\hline QoE requirement & max-SINR & multiple association \\
\hline $100 \%$ coverage & $3 \mathrm{VNs}$ & $9 \mathrm{VNs}$ \\
$98 \%$ coverage & $4 \mathrm{VNs}$ & $11 \mathrm{VNs}$ \\
$88 \%$ coverage & $6 \mathrm{VNs}$ & $14 \mathrm{VNs}$ \\
\hline
\end{tabular}

Table 9: The impact of traffic spatial heterogeneity

\begin{tabular}{|l|l|l|l|}
\hline Traffic Type & $\mathbf{1 0 0 \%}$ coverage & $\mathbf{9 8 \%}$ coverage & $\mathbf{8 8 \%}$ coverage \\
\hline PPP & $9 \mathrm{VNs}$ & $11 \mathrm{VNs}$ & $14 \mathrm{VNs}$ \\
Matern & 4 to $12 \mathrm{VNs}$ & 5 to $14 \mathrm{VNs}$ & 6 to $16 \mathrm{VNs}$ \\
\hline
\end{tabular}

for multiple association versus max-SINR association.

One of the advantages of our proposed VN admission control method is that the customer traffic specification is not limited and can be any distribution with concave CDF. As a main result, the customer traffic profiles can be heterogeneous. In this section, we investigate the impact of traffic heterogeneity on the VN admission control results.

We generate two different traffic profiles. The first profile is a homogeneous PPP with constant density all over the network while the second profile is a clustered distribution with the same total density. In the clustered distribution, a Matern-like distribution [90] is applied. First, we select a number of cluster center locations which are uniformly distributed in the network. Then, the bins around the cluster centers (with certain radius) have higher density while the other bins have low or zero density.

Table 9 summarizes the number of VNs with certain coverage requirements for homogeneous PPP versus Matern heterogeneous PPP with 10 cluster centers. As expected, the number of admitted VNs in a network with heterogeneous traffic depends on the cluster locations and varies in a range. If the clusters shape near BSs, then the network performance increases and if the clusters shape far from BSs (at cell edges), then the network performance decreases.

Two main parameters can be changed to illustrate the impact of traffic heterogeneity on the admission control process: 
Table 10: The impact of traffic correlation with BSs $(\mathrm{CoV}=2)$

\begin{tabular}{|l|l|l|l|}
\hline$\rho$ & $\mathbf{1 0 0 \%}$ coverage & $\mathbf{9 8 \%}$ coverage & $\mathbf{8 8 \%}$ coverage \\
\hline-0.5 & $7 \mathrm{VNs}$ & $9 \mathrm{VNs}$ & $11 \mathrm{VNs}$ \\
0 & $9 \mathrm{VNS}$ & $11 \mathrm{VNs}$ & $14 \mathrm{VNs}$ \\
+0.5 & $10 \mathrm{VNs}$ & $13 \mathrm{VNs}$ & $15 \mathrm{VNs}$ \\
\hline
\end{tabular}

Table 11: The impact of traffic correlation with BSs $(\mathrm{CoV}=3)$

\begin{tabular}{|l|l|l|l|}
\hline$\rho$ & $\mathbf{1 0 0 \%}$ coverage & $\mathbf{9 8 \%}$ coverage & $\mathbf{8 8 \%}$ coverage \\
\hline-0.5 & $5 \mathrm{VNs}$ & $8 \mathrm{VNs}$ & $10 \mathrm{VNs}$ \\
0 & $9 \mathrm{VNs}$ & $11 \mathrm{VNs}$ & $14 \mathrm{VNs}$ \\
+0.5 & $11 \mathrm{VNs}$ & $14 \mathrm{VNs}$ & $16 \mathrm{VNs}$ \\
\hline
\end{tabular}

- The first parameter is the level of heterogeneity of traffic. This can be captured by many metrics including coefficient of variation (CoV) introduced and explained in [26] and [28]. CoV value of 0 indicates complete homogeneity and $\mathrm{CoV}$ value of 1 indicates complete randomness. The higher $\mathrm{CoV}$ value shows more heterogeneity.

- The second parameter is the correlation between UE traffic and BSs $(\rho)$ which ranges from -1 (where all UEs are at the cell edges) to +1 (where all UEs are at the cell centers) [28].

In the next experiment, the UE clusters are changed to show the effect of traffic correlation to BSs. Tables 10, 11, and 12 show the maximum number of admitted $\mathrm{VNs}$ with various coverage requirements and correlation between traffic and BSs for different $\mathrm{CoV}$ values.

Finally, the last experiment is to capture the effect of backhaul limits on the performance and the number of VNs which can be admitted with certain coverage requirements. For this purpose, we decrease the backhaul limits of the pico-BSs and leave the backhaul limits of macro-BSs to be unlimited. As expected, the number of admitted VNs goes down with decreasing the backhaul limits. Figure 35 illustrates the maximum number of admitted VNs versus the capacity of the backhaul links of 
Table 12: The impact of traffic correlation with BSs $(\mathrm{CoV}=4)$

\begin{tabular}{|l|l|l|l|}
\hline$\rho$ & $\mathbf{1 0 0 \%}$ coverage & $\mathbf{9 8 \%}$ coverage & $\mathbf{8 8 \%}$ coverage \\
\hline-0.5 & $4 \mathrm{VNs}$ & $7 \mathrm{VNs}$ & $8 \mathrm{VNs}$ \\
0 & $9 \mathrm{VNs}$ & $11 \mathrm{VNs}$ & $14 \mathrm{VNs}$ \\
+0.5 & $12 \mathrm{VNs}$ & $15 \mathrm{VNs}$ & $17 \mathrm{VNs}$ \\
\hline
\end{tabular}

the pico-BSs.

\subsection{Conclusion}

We proposed an analytical solution for virtual network admission control problem in future software-defined wireless networks. In particular, we presented an admission control procedure which includes a feedback mechanism to correct customer traffic information. We also proposed an optimization framework for virtual network admission control which allows various options and flexibility for specification of customer traffic and considers all important QoE parameters including rate, delay, and outage. We also proposed two extensions to consider network cost and backhaul limitations in the problem.

The simulation results revel a number of interesting observations:

- first, the simulation results and the expected analytical results are closely matched. This shows that the optimization method provides accurate solutions and can be used to insure customer QoE as well as high network utilization.

- secondly, the multiple association method out-performs the best rate single association method and the max-SINR association method.

- finally, the heterogeneity in VN demand profiles results in a wide range of admission scenarios. In the cases where the UE clusters are close to the BSs, a higher number of VNs can be admitted; while in the cases where the UE clusters emerge in cell edges, less VNs with the same requirements can be admitted into the system.

This work can be extended in many directions. First, the power control is not considered in our method and we assumed that transmit power is constant. However, 


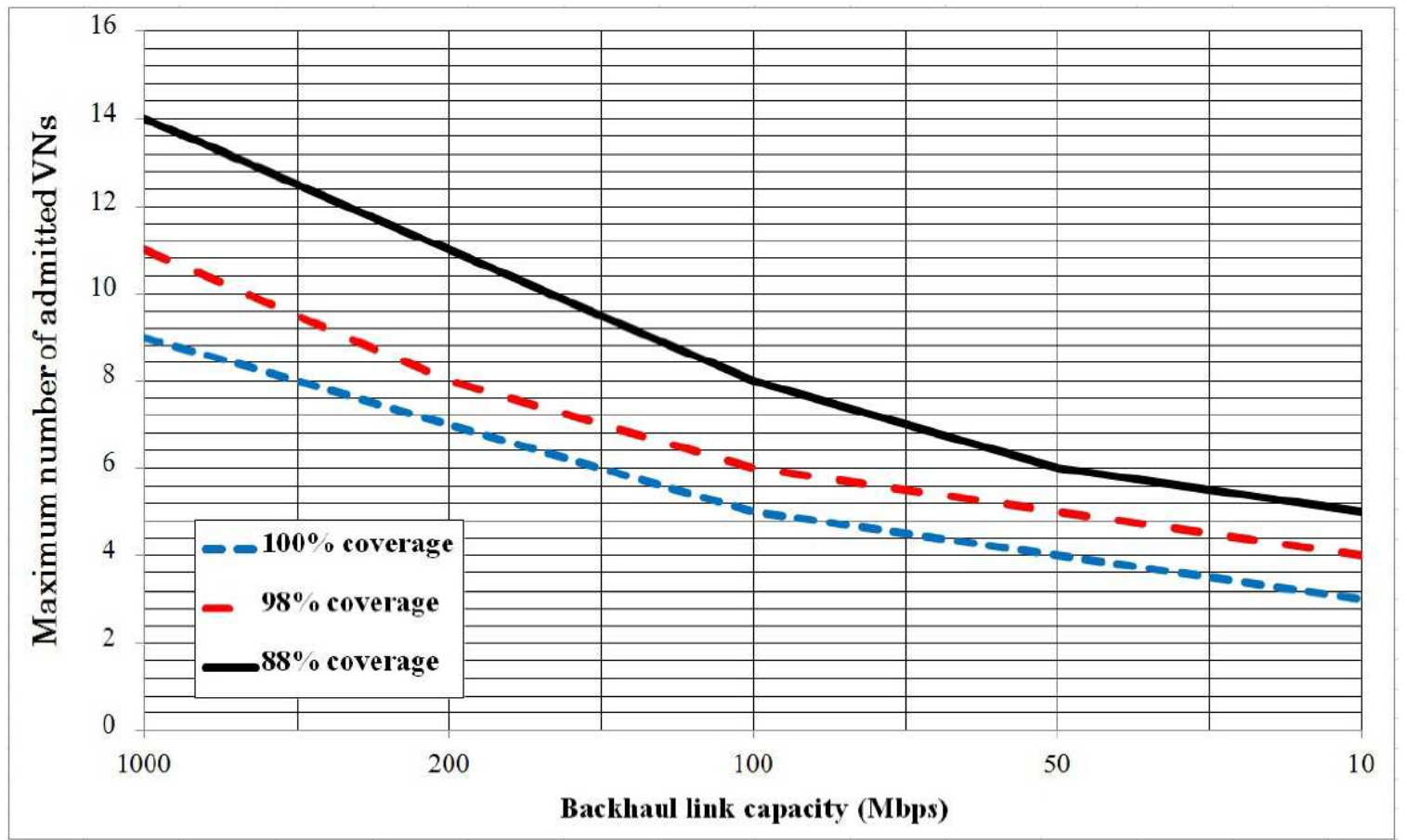

Figure 35: The figure shows the number of admitted VNs in backhaul limited scenarios. As expected, the number of admitted VNs goes down with decreasing the backhaul limits. 
power control can increase the network performance, hence the number of admitted VNs. Secondly, we assumed that all BSs transmit on all channels and there is no interference control. Adding inter-cell interference coordination (ICIC) improves the network performance and can be considered as an extension of this work. 


\section{Chapter 6}

\section{VNAC with Joint Backhaul and Access Optimization}

\subsection{Introduction}

The future $5 \mathrm{G} / 5 \mathrm{G}+$ multi-service wireless networks must incorporate not only shortterm single-session requests by individual users, but also long-term virtual network (VN) service requests by groups of users. Indeed, user groups with statistical distribution of traffic demands and specific quality-of-service (QoS) requirements submit requests for $\mathrm{VN}$ services to the service provider, and the service provider, considering its limited resources and capabilities, must decide whether to accept or reject the requests. This is performed in the admission control module of the service provider's network operating system.

Admission control is performed based on a large number of parameters including the customer traffic profile (user equipment (UE) locations), available bandwidth at base stations (BSs), and customer QoS requirements. The virtual network admission control in a wireless network is different from (and more complicated than) the singlesession admission control. In the single-session admission control, decision is made based on a (deterministic) snapshot of already existing UE locations and the new UE location; while in the VN admission control, the UE locations and traffic demand distribution information are provided statistically by the VN customers. For instance, VN customers might indicate the expected distribution of traffic in various areas of the network. Therefore, the VN requests must be admitted if their QoS can be satisfied statistically. After (long-term) admission of a VN, (short-term) single-sessions of the 


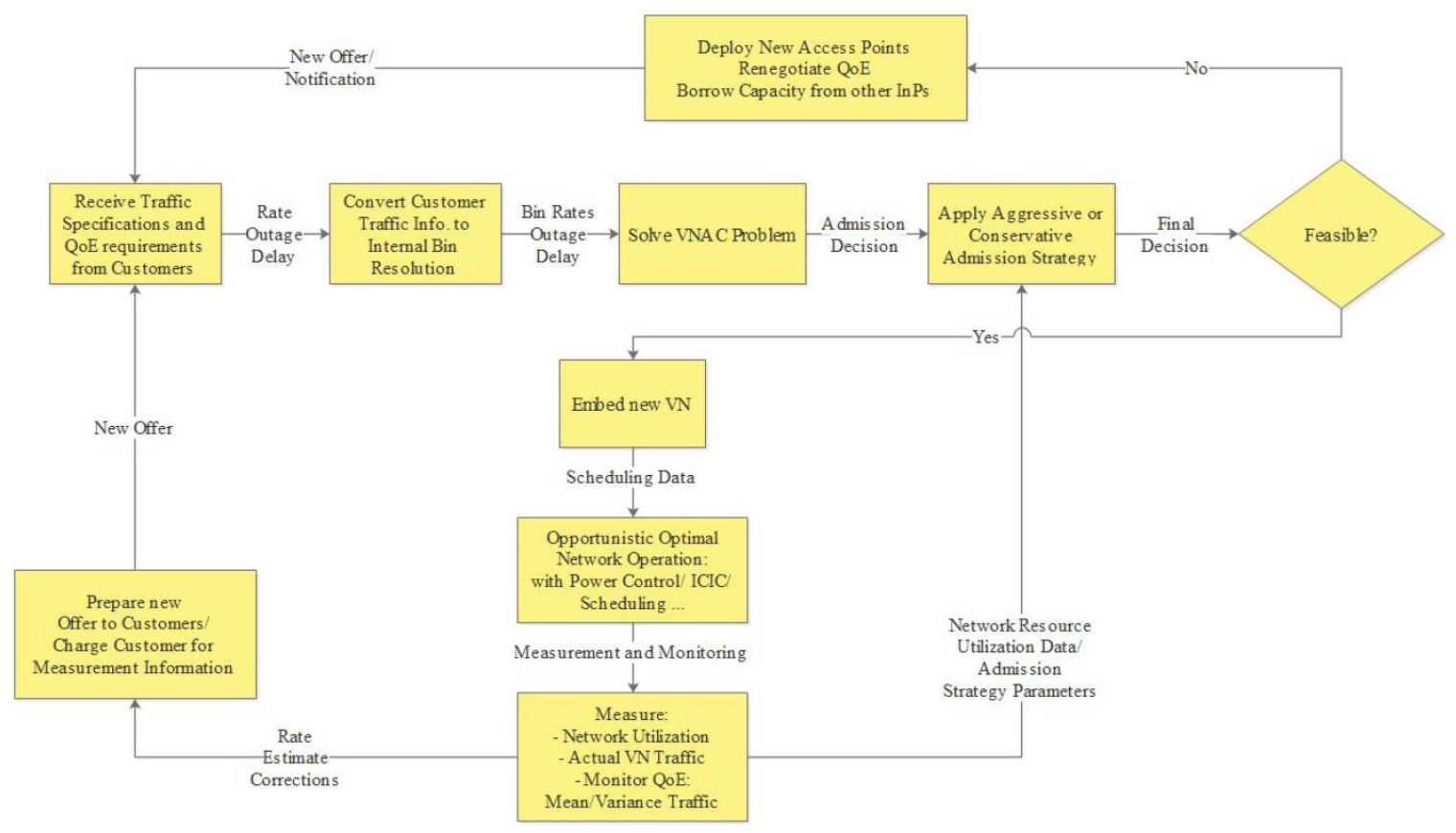

Figure 36: Admission control process: admission decision is based on VN service requests and available resources.

VN users are admitted only if they follow the restrictions and limitations of the VN admission contract.

In service-based networks, in addition to QoS requirements, services may require processing at certain nodes in a specific order. Such requirements are referred to as service function chain (SFC) requirements. Traffic aggregation, caching, encryption, and video trans-coding are examples of service functions. Different services may have different SFC requirements. However, a service function can be common to different services, e.g., the contents of web services and video services may all be cached at the same node. A network node where a service function is instantiated at is called a service function node ( $\mathrm{SFN})$.

The most common approach to solve the network problems with traversal constraints is to parse flows into flow segments according to their traversal constraints and apply the traditional multi-commodity flow (MCF) problem formulation [91] with flow segments as commodities. Such approach does not scale with increasing number of flows/flow segments both in terms of admission control algorithm complexity and the memory requirements. 
On the other hand, in a wireless cellular network with ideal backhaul connections, the lack of capacity is mainly due to the access connection limitation, that is, the wireless access problem and low channel quality between UEs and BSs. However, in the envisioned 5G networks with small cells deployed in residential and office buildings, the assumption of ideal backhaul is more optimistic than realistic, especially considering the fact that a main portion of the small cells (i.e., femtocells) will be deployed by customers rather than service providers. Recently, there has been increasing interest in the literature in the study of limited backhaul capacities in future $5 \mathrm{G}$ HetNets. Figure 36 illustrates the admission control process and its main input parameters.

In this chapter, we propose an efficient, tractable, and scalable service-segmentbased admission control method for wireless VNs which incorporates network access limitations, network backhaul limitations, and service function limitations. The main contributions of this chapter are summarized as follows:

- A novel wireless VN admission control method is proposed which incorporates both the access limitations and backhaul limitations in admission decision for general network topologies.

- This problem is formulated as a joint convex optimization problem which is computationally tractable.

- Service segments and SFC constraints are included in the problem both in the sense of service function orders and the resource requirements at each service function node.

- A service-segment-based approach is put forth which is significantly more scalable compared to its flow-segment-based counterpart.

Admission control is a very well investigated concept and there is a great volume of literature focusing on various aspects of admission control [30-33]. However, since the idea of network virtualization is relatively new [34-36], admission control for VN requests, also referred to as $\mathrm{VN}$ embedding, has been a hot research topic recently as in articles [37-40]. Indeed, admission control for single users and single sessions is studied very well but the admission control of groups of users and VN requests is relatively recent. 


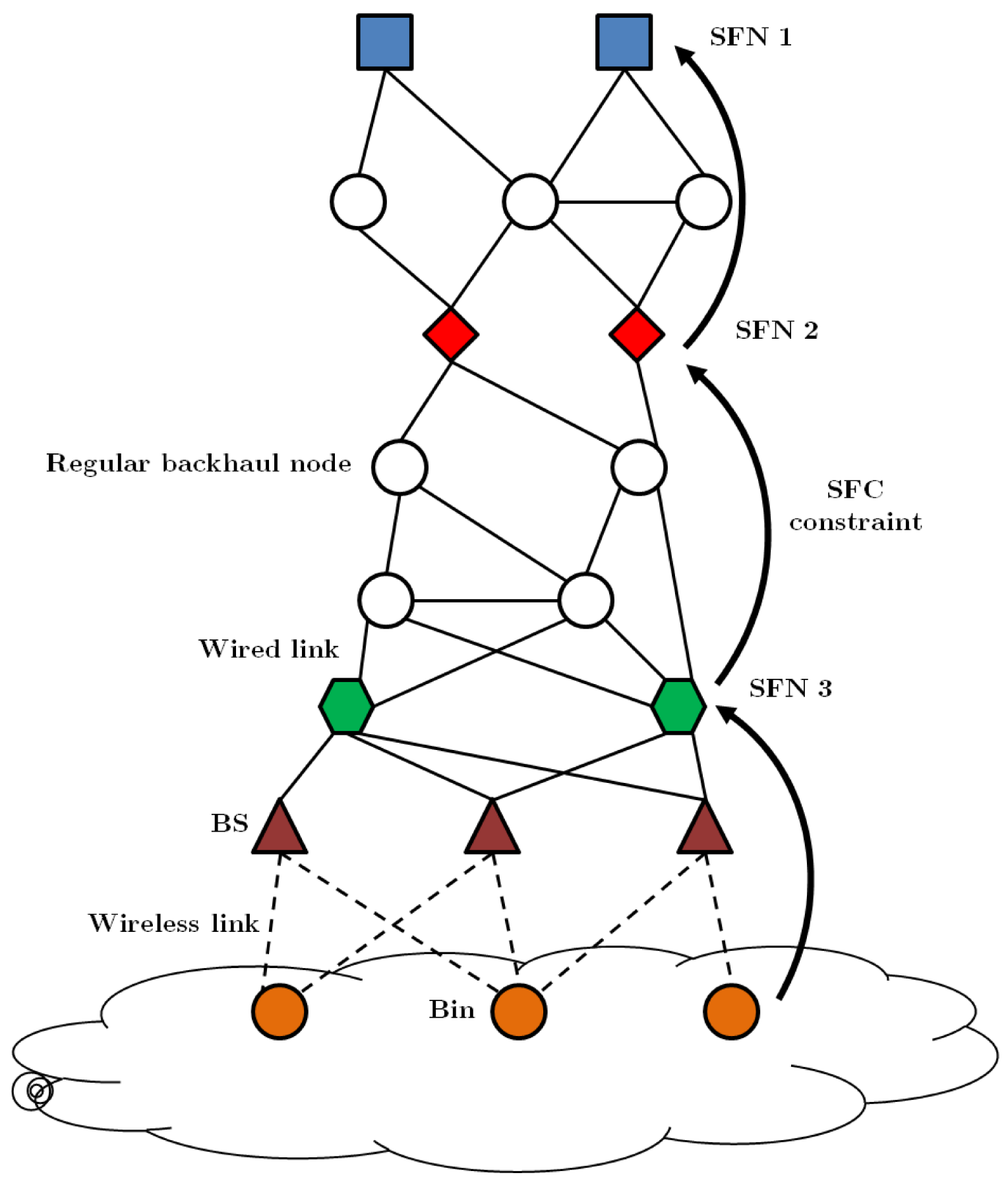

Figure 37: We consider a wireless cellular network with general access and backhaul topologies and SFC constraints. 
Although there is a rich literature on VN admission control in wired networks, there are only few works on wireless VN admission control [41-46]. In articles [41] and [42], fixed snapshots of UEs are considered, but the statistical specifications of traffic demand are not incorporated. In [43-46], the authors consider statistical arrival of VN requests with statistical demands. However, the demand and the resources are not specified in rate or delay, but rather in number of channels. To be more accurate, these papers assume that customers require a specific number of wireless channels, and the network allocates a number of channels to each UE upon availability, i.e., the spectral efficiency is not included in the allocation scheme and rate satisfaction is not the ultimate objective in these works.

The remaining of this chapter is organized as follows. In Section 6.2, the system model is described and the $\mathrm{VN}$ admission control problem with joint optimization is introduced. Sections 6.3 and 6.4, present the proposed flow-based and segment-based methods for VN admission control, respectively. The simulation results are presented in Section 6.5. The chapter is concluded in Section 6.6.

\subsection{System Model and Problem Definition}

We consider the downlink of a cellular wireless network with general access and backhaul topologies. A geographical region $Q \subset \mathbb{R}^{2}$ (i.e., two dimensional plane) is assumed as the network layout that is served by a set of BSs $B$. Each BS is characterized by its available bandwidth $W_{k}, k \in K=\{1, \ldots,|B|\}$. Let $q \in Q$ denote a location on the network layout. We assume that demand arrivals for the $i$ th $\mathrm{VN}$ service, $v_{i} \in V$, at location $q, d_{i q}$, are random variables that follow arbitrary distributions with mean $\lambda_{i q}$, where $V$ is the set of $\mathrm{VN}$ services. This provides flexibility for inhomogeneous, i.e., heterogeneous or non-uniform, traffic characterization. To avoid unnecessary technical difficulties, we assume that the region $Q$ is divided into a set of small (e.g., $5 \mathrm{~m} \times 5 \mathrm{~m}$ ) bins (areas) $a \in A$, and the demand for each bin is defined as

$$
d_{i j}=\int_{q \in a_{j}} d_{i q}, j \in J, i \in I
$$

where $J=\{1, \ldots,|A|\}$ and $I=\{1, \ldots,|V|\}$.

We assume that the BSs transmit with constant (not necessarily equal) power; we leave power control extension as a future work. The signal-to-interference-plus-noise 
ratio (SINR) for the center of bin $a_{j} \in A$ if it is connected to BS $b_{k} \in B$ is defined as

$$
\gamma_{j k}=\frac{P_{j k}}{P_{N}+\sum_{j^{\prime} \in J j} P_{j^{\prime} k}},
$$

where $P_{j k}$ is the effective received power from BS $b_{k}, k \in K$ at bin $a_{j}$, and $P_{N}$ is the noise power.

The received power can be calculated based on path-loss exponent and channel models as well as the other environmental parameters. In practical cases, it also can be collected from field measurements. Spectral efficiency (SE) can be any arbitrary function of SINR in this method. In our simulations, we assume that it follows the Shannon's formula as follows:

$$
\eta_{j k}=\log _{2}\left(1+\gamma_{j k}\right)
$$

While the simplest (and mostly used) UE-BS association is based on maximum received power and maximum SINR (max-SINR), technology advances in future wireless networks allow for general multiple association in which a UE can be associated to multiple BSs, or to a BS with low SINR but high available bandwidth. It is shown in the recent literature [82-84] that general association can result in significant improvement in network key parameter indicators, specially in future HetNets where small cells have limited coverage areas hence lower load compared to macro cells. Therefore, the $\mathrm{VN}$ admission control method proposed in this chapter allows for general UE-BS association.

We assume that QoS expectations are specified by each VN customer by two main parameters: the required traffic demand which is specified by the traffic demand matrix $d$, or more specifically, by the distribution parameters of traffic in each bin, and the required maximum outage $O_{i}$. In addition, the SFC order and service function resource requirements are specified by each VN service customer. Figure 37 shows a sample network with access, backhaul, and SFNs.

The backhaul network is modeled as a directional graph $G(N, L)$, where $N$ and $L$ are the sets of nodes and links in the network, respectively. Let $L_{\text {in }}(n)$ and $L_{\text {out }}(n)$ be the sets of links terminating and originating at node $n \in N$, respectively. There are multiple services present in the network. There is a service function chain associated with each service. A service function chain is an ordered sequence of (service function) nodes in G. We consider a service as a set of flows with some common SFC 


\section{Original flow}

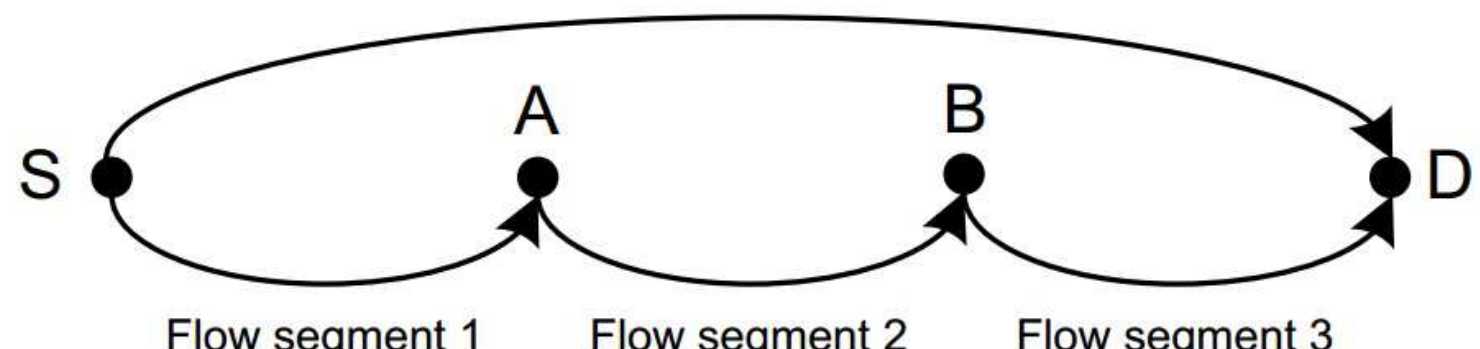

Figure 38: Flow segmentation: the original flow is parsed into smaller flow segments based on the SFC constraints.

requirements, i.e., all flows within a service have some common traversal constraints. In the case of multiple deployment/instantiation of the same service function at network nodes, we assume fixed association of service flows to one of the multiple service function nodes.

We denote a flow with traversal constraint with $S \rightarrow N_{1} \rightarrow \cdots \rightarrow N_{m} \rightarrow D$ where $S$ and $D$ are the flow source and destination, respectively, and $N_{1}, \ldots, N_{m}$ are intermediate nodes that the flow must visit in the specified order before reaching its destination. Accordingly, a service is denoted by $S \rightarrow N_{1} \rightarrow \cdots \rightarrow N_{m} \rightarrow D$, where $S$ is the set of source nodes of flows within the service and $D$ is the set of destinations of flows within the service.

\subsection{Flow-Based VN Admission Control}

In this section, a flow-segment-based admission control approach is described.

\subsubsection{Flow Segmentation}

In order to address the flow traversal constraints, each flow is parsed according to its traversal constraints into multiple flow segments. This approach is illustrated in Figure 38 for a flow which must go through service function nodes $A$ and $B$ before reaching its destination. 


\subsubsection{Flow-Segment-Based VN Admission Control}

A flow-based admission control method for $\mathrm{VN}$ requests can be applied to address the flow traversal constraints by using flow-segments as commodities. In other words, in the flow-segment-based admission control, the flow commodities are flow segments rather than the original flows. The demand of each flow segment is equal to the corresponding original flow.

For a flow-segment-based admission control method, first, note that VN service admission control problem is a feasibility problem in which we are not trying to improve an objective function as the ultimate goal is to determine if some admission control constraints can be satisfied or not. Let $x_{i j k}$ denote the amount of rate received from BS $b_{k} \in B$ at bin $a_{j} \in A$ by VN $v_{i} \in V$. We call this flow $f_{i j}$. Also let $z_{i j l}$ denote the amount of traffic for flow $f_{i j}$ passing through link $l \in L$. The objective of the optimization is to find a solution $x^{*}$ and $z^{*}$ that satisfies optimization constraints.

The optimization constraints can be divided into two categories: the access constraints, and the backhaul constraints. First, the access constraints are presented and then the backhaul constraints follow.

The first constraint is that the sum of the resources allocated by each BS to all bins cannot exceed its available resources:

$$
\mathrm{C} 1: \quad M_{k}=\sum_{i \in I} \sum_{j \in J} \frac{x_{i j k}}{\eta_{j k}} \leqslant W_{k}, \forall k \in K
$$

where $M_{k}$ is the amount of used resources from BS $k, W_{k}$ is the available resources, and $\eta_{j k}$ is the effective spectral efficiency experienced from BS $k$ at bin $j$.

Secondly, the outage constraint is that outage probability for all customers must be less than the maximum allowed outage. The outage probability for a $\operatorname{VN} v_{i} \in V$ for one bin $a_{j} \in A$ is equal to the probability that the demand for this $\mathrm{VN}$ in this bin is higher than its received rate from all BSs:

$$
\xi_{i j}=\operatorname{Pr}\left\{d_{i j} \geqslant r_{i j}\right\}
$$

where $r_{i j}$ is the total rate received by $\operatorname{VN} v_{i} \in V$ at bin $a_{j} \in A$ :

$$
\mathrm{C} 2: \quad r_{i j}=\sum_{k \in K} x_{i j k}, \forall i \in I, \forall j \in J
$$


To calculate the outage probability for a VN over the entire network, one way is to take the normal average. However, since different bins have different traffic demands, a better measure is a weighted average:

$$
\xi_{i}=\frac{1}{\lambda_{i}}\left(\sum_{j \in J} \lambda_{i j} \cdot \xi_{i j}\right)
$$

where $\lambda_{i}=\sum_{j \in J} \lambda_{i j}$ is the total demand for $\mathrm{VN} v_{i} \in V$. Therefore, the outage constraint is

$$
\text { C3: } \quad \xi_{i} \leqslant O_{i}, \forall i \in I
$$

The service function requirements at each SFN can also be considered in the optimization problem. Let $H$ be the set of all SFNs in the network. Also assume that each VN service $v_{i}$ requires $u_{i h}$ resource usage at $\mathrm{SFN} h \in H$. The SFN resource constraint can be stated as

$$
\text { C4: } \quad \sum_{i \in I} u_{i h} \leqslant U_{h}, \forall h \in H
$$

where $U_{h}$ is the total resources available at $\operatorname{SFN} h \in H$.

The first backhaul constraint is that the flow conservation law must hold at all network nodes:

$$
\text { C5: } \quad \sum_{l \in L_{\text {in }}(n)} z_{i j l}=\sum_{l \in L_{\text {out }}(n)} z_{i j l}, \forall n \in N, \forall i \in I, \forall j \in J .
$$

For the BSs, since they are at the border between backhaul and access, the flow conservation law is stated as follows:

$$
\text { C6: } \quad \sum_{l \in L_{i n}(k)} z_{i j l}=x_{i j k}, \forall k \in K, \forall i \in I, \forall j \in J \text {. }
$$

Finally, the last backhaul constraint is that the total traffic passing through each link cannot exceed the link capacity:

$$
\mathrm{C} 7: \quad \sum_{i \in I} \sum_{j \in J} z_{i j l} \leqslant C_{l}, \forall l \in L
$$

where $C$ is the link capacity matrix. 
In summary, the flow-segment-based VN admission control problem can be cast as the following problem:

$$
\text { find }_{x, z} x, z \text { subject to: } \mathrm{C} 1-\mathrm{C} 7 \text {. }
$$

\subsection{Service-Based VN Admission Control}

The flow-based approach described in Section 6.3 does not scale with increasing number of flows/flow segments in terms of admission control algorithm complexity. In this section, we propose a low-complexity admission control approach by taking advantage of the property that all flows within a service have the same traversal constraints.

\subsubsection{Service Segmentation}

We parse flows according to their SFC requirements into flow segments as described in Section 6.3.1. A service segment is defined as a set of flow segments with common source node. Alternative definitions of service segment may be considered in different problems. For instance, in a network problem which is involved in uplink streams, a service segment can be defined to be a set of flows with common destination. However, in our problem, since we are considering downlink, defining service segments based on common source nodes helps to reduce the problem size. Figure 39 illustrates the bundling of flow segments with common source nodes under service segments.

We assume that all of the traffic for each VN service goes through the same service chain. In other words, each VN service has a specific SFC. Therefore, the set of service segments matches the VN service segments.

\subsubsection{Service-Segment-Based VN Admission Control}

The service-segment-based admission control optimization formulation is presented in this section. The access constraints remain the same as flow-segment-based method due to the fact that the QoS requirements of $\mathrm{VN}$ services must be satisfied in a per bin basis. However, the backhaul constraints for the service-segment-based admission control are highly efficient and more scalable than the flow-segment-based method. Indeed, the number of optimization variables is decreased considerably. 


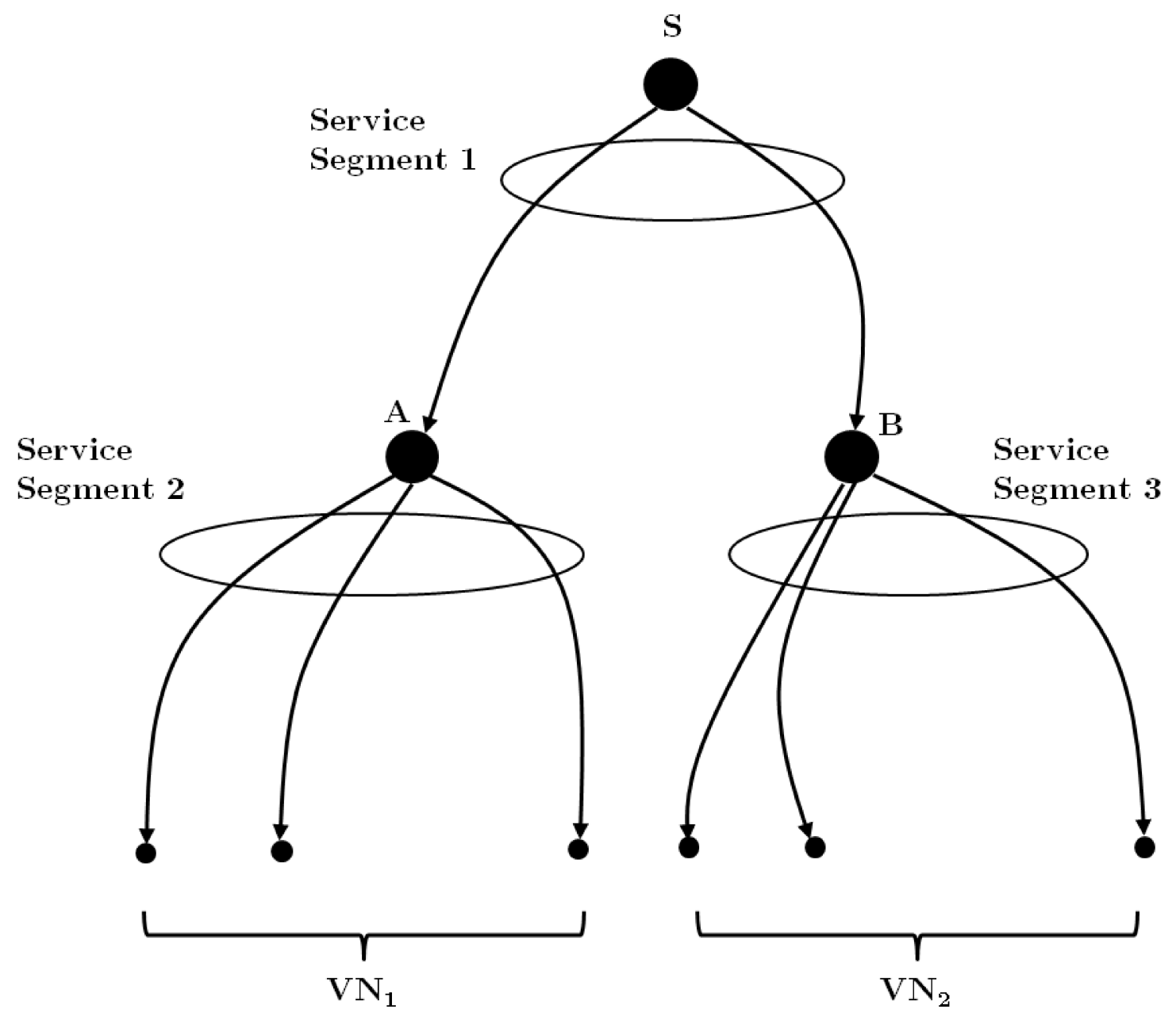

Figure 39: Flow segments with common source node are categorized under a service segment. 
Table 13: Comparison between flow-based and service-based methods in terms of number of variables and constraints.

\begin{tabular}{l|l|l}
\hline Criteria & Flow-based & Service-based \\
\hline Var. & $|I| \times|J| \times(|K|+|L|)$ & $|I| \times|J| \times|K|+|L|$ \\
\hline Cons. & $\alpha+|I| \times|J| \times|N|$ & $\alpha+|N|$ \\
\hline
\end{tabular}

First, note that the constraint (86) will change as follows:

$$
\text { C8: } \quad \sum_{l \in L_{\text {in }}(n)} y_{l}=\sum_{l \in L_{\text {out }}(n)} y_{l}, \forall n \in N
$$

where $y_{l}$ represents the total amount of traffic passing through link $l$. It is clear that the size of variable $y$, compared to $z$ in flow-based method, is reduced by a $|I| \times|J|$ magnitude.

Secondly, the constraint (87) can be stated in a summarized form as follows:

$$
\text { C9: } \quad \sum_{l \in L_{i n}(k)} y_{l}=x_{i j k}, \forall k \in K, \forall i \in I, \forall j \in J
$$

Finally, the constraint (88) is transformed in a very simple inequality as

$$
\text { C10: } \quad y_{l} \leqslant C_{l}, \forall l \in L
$$

Therefore, the service-segment-based admission control optimization problem can be stated as follows:

$$
\text { find }_{x, y} x, y \text { subject to: } \mathrm{C} 1-\mathrm{C} 4, \mathrm{C} 8-\mathrm{C} 10 \text {. }
$$

It can be shown easily that (93) has a significantly lower number of optimization variables and optimization constraints compared to (89). Table 13 shows the number of optimization variables and constraints in both flow-based and service-based admission control methods. In Table 13, $\alpha$ is defined as

$$
\alpha=|K|+|I| \times|J|+|I|+|I| \times|J| \times|K|+|L| .
$$




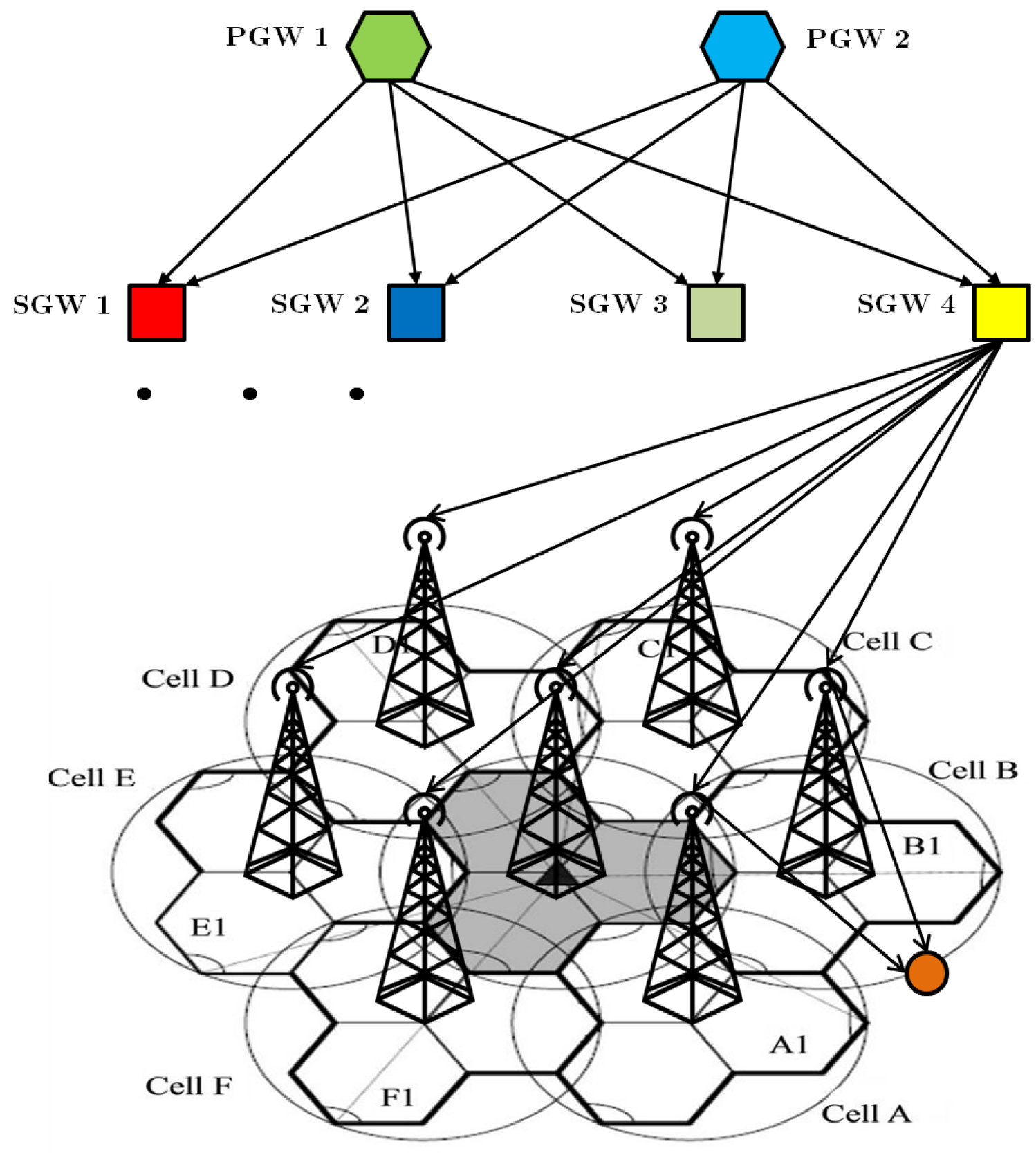

Figure 40: We consider a network with 2 PGWs, 4 SGWs, 7 BSs, and 21 sectors. 


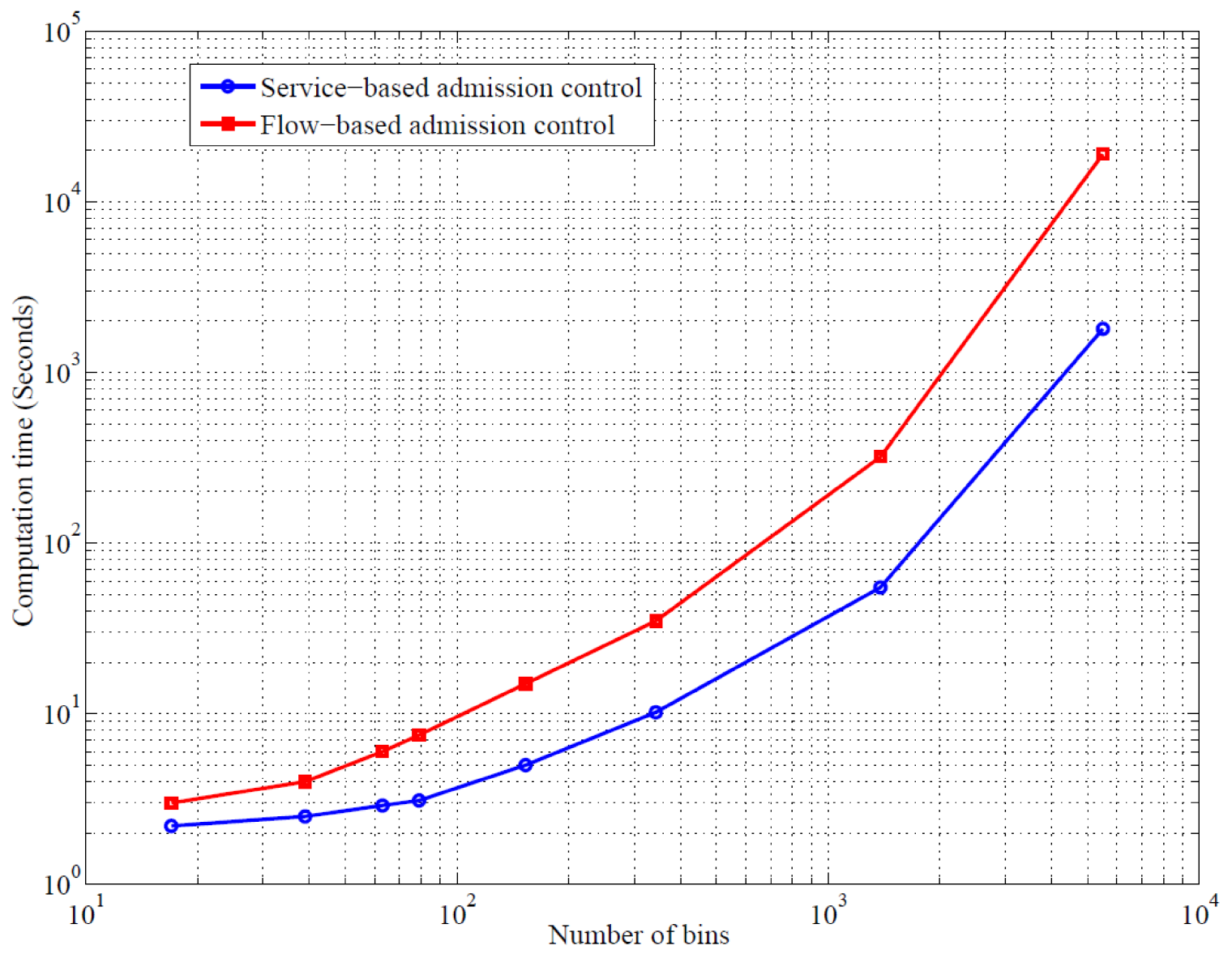

Figure 41: Computation time: the vertical axis shows the computation times for flowbased and service-based admission control algorithms. The horizontal axis shows the number of flows. 


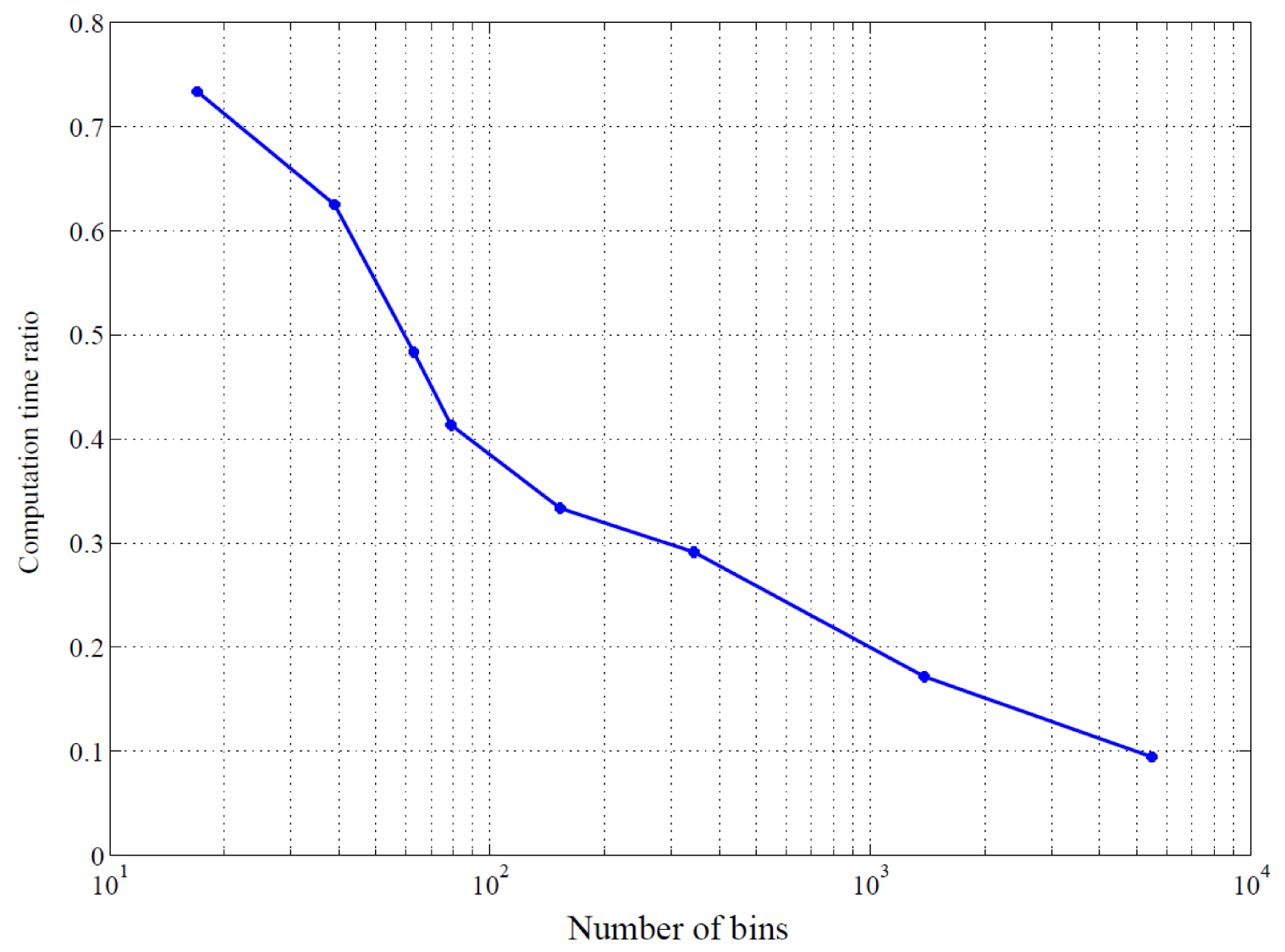

Figure 42: Computation time ratio: the ratio of computation time of flow-based admission control algorithm to the computation time of service-based algorithm is illustrated versus the number of flows.

\subsection{Simulation Results}

In this section, we compare admission control algorithm computation times as a measure of complexity for service-based and flow-based formulations. We also verify through simulation that service-based approach incurs no loss in optimality.

\subsubsection{Simulation Setup}

The proposed methods in this chapter are general and can be applied to general access network and backhaul network topologies. In our simulation we consider the network topology illustrated in Figure 40. We assume that there are $4 \mathrm{VN}$ service requests each to be served by a service gateway (SGW) and a packet gateway (PGW). The 
SGWs perform a first level of aggregation on the VN data and the PGWs perform a second level of aggregation on top of that.

There are 4 SGWs and 2 PGWs in the network backhaul. Each VN has a dedicated SGW. VNs $v_{1}$ and $v_{2}$ are served by PGW1 and VNs $v_{3}$ and $v_{4}$ are served by PGW2. The access network comprises 7 BSs each having 3 sectors, 21 sectors in total. All BSs have full connection with all SGWs and all SGWs have two connections to the PGWs. All BSs have $10 \mathrm{MHz}$ bandwidth available for serving VN requests. We assume that the outage requirement for all VNs is 2 percent. Noise power is assumed to be -174 dB.

\subsubsection{Complexity Comparison}

We use the admission control algorithm computation times as a measure of computational complexity. Figures 41 and 42 compare the running times of service-based and flow-based algorithms as a function of the number of flows in the network. Figure 41 shows the actual running times, in log scale, for both schemes as a function of the number of flows. Figure 42 shows the ratio of service-based running time to flow-based running time as a function of the number of flows. According to Figures 41 and 42, with more than 5000 flows in the network, the service-based algorithm is about 11 times faster than the flow-based algorithm. The problem complexity increases polynomially with the number of flows. However, the complexity of service-based does not increase significantly as the number of flows increases. This is due to the fact that the number of service segments is fixed in the network backhaul and does not increase as the number of flows increases. Furthermore, the number of backhaul constraints in the service-based admission control problem does not change as the number of flows increases.

\subsection{Conclusion}

In this chapter, we considered the problem of admission control of VN service requests in a multi-service network. We formulated the problem as a joint optimization of access and backhaul which is also convex and tractable. We showed that a service-based formulation can reduce the problem size which leads to reduction of computation time and number of variables and constraints. 


\section{Chapter 7}

\section{Conclusions and Future Work}

\subsection{Conclusions and Contributions}

The work described in this thesis has been concerned with traffic modeling in wireless networks. Considering the fact that in the envisioned $5 \mathrm{G}$ wireless cellular networks traffic is expected to be heterogeneous in both supply and demand while an enormous majority of the existing literature on traffic modeling in wireless cellular networks consider only homogeneous traffic scenarios, in this thesis, we proposed an accurate, realistic, simple, and adjustable modeling for the future heterogeneous wireless cellular networks with heterogeneous traffic distributions.

In Chapter II, we proposed a traffic modeling process describing a systematic approach to traffic modeling. A new statistical approach for modeling the spatial traffic in heterogeneous cellular networks was introduced. A number of novel distance-based traffic measures in space were proposed which can be considered as the equivalents of the $i a t$ in the time domain. Only two statistical parameters were used to regulate the heterogeneity and bias of traffic towards the BSs. Stochastic geometry was used to generate realistic and adjustable traffic. The effects of the realistic traffic modeling on the performance of heterogeneous wireless cellular networks was illustrated. According to the proposed process, we introduced a traffic modeling in which the heterogeneity of the UE distribution as well as the correlation between UEs and BSs are adjustable.

In Chapter III, we put forth an analytical modeling for SIR, coverage and other metrics in HetHetNets showing the impact of the traffic heterogeneity and the UE-BS correlation on the performance of HetHetNets. In this chapter, we derived closed-form 
analytical models for SIR and coverage in wireless cellular networks with heterogeneous traffic.

Chapter IV demonstrated how the UIL technique can take heterogeneity into account for finding the best location to suggest to the user for moving. We introduced improved UIL schemes whereby the BS loads and the BS backhaul connections are taken into account. The simulation results confirm that the new UIL scheme improves such important network performance metrics as the mean user rate and the coverage probabilities in the future $5 \mathrm{G}$ networks.

In Chapters V and VI, we presented algorithms and applications in admission control of VNs in wireless networks which can exploit this realistic traffic modeling to enhance the network performance. We proposed an analytical solution for virtual network admission control problem in future software-defined wireless networks. In particular, we presented an admission control procedure which includes a feedback mechanism to correct customer traffic information. We also proposed an optimization framework for virtual network admission control which allows various options and flexibility for specification of customer traffic and considers all important QoE parameters including rate, delay, and outage. We also proposed two extensions to consider network cost and backhaul limitations in the problem. We considered the problem of admission control of VN service requests in a multi-service network. We formulated the problem as a joint optimization of access and backhaul which is also convex and tractable. We showed that a service-based formulation can reduce the problem size which leads to reduction of computation time and number of variables and constraints.

\subsection{Future Works}

The traffic modeling method presented in Chapter II is general enough to be applied in other wireless networks as well. For instance, the relaying and routing problems in ad hoc and Wi-Fi networks are closely related, and are dependent on the distribution of the network terminals. In wireless networks, the cell switch-off framework is also highly dependent on the spatial distribution of the traffic.

With the emergence of HetNets, antenna height might also be different for various types of base station. While Micro-BSs might be installed on top of skyscrapers, Pico-cells are generally installed in lower heights and femto-cells are installed by 
customers inside buildings and in many different heights. As such, a 3D point pattern representing BSs and users can help in a more accurate modeling of a future wireless network. This is a very promising and important extension of this $\mathrm{PhD}$ work.

It is interesting to note that various characteristics of the user distributions in wireless networks can be observed in other phenomena as well. For instance, the similar problems of heterogeneity of distribution or self-similarity arise in micro-cosmic level investigations in chemistry and particle physics and in macro-cosmic level studies in astrophysics.

Chapter III also can be extended in many directions. First, other modeling approaches might be presented which result in more accurate expressions. Moreover, closed-form expressions for other metrics such as UE rates could be derived.

VNAC presented in Chapters V and IV can be extended in many directions. First, the power control is not considered in our method and we assumed that transmit power is constant. However, power control can increase the network performance, hence the number of admitted VNs. Secondly, we assumed that all BSs transmit on all channels and there is no interference control. Adding inter-cell interference coordination improves the network performance and can be considered as an extension of this work. 


\section{Bibliography}

[1] J. G. Andrews, S. Buzzi, W. Choi, S. V. Hanly, A. Lozano, A. C. Soong, and J. C. Zhang, "What will 5G be?," IEEE Journal on Selected Areas in Communications, vol. 32, pp. 1065-1082, June 2014.

[2] L. Atzori, A. Iera, and G. Morabito, "The Internet of things: A survey," Computer Networks, vol. 54, pp. 2787-2805, October 2010.

[3] ITU-R., "ITU-R M.2135: Guidelines for Evaluation of Radio Interface Technologies for IMT-Advanced," tech. rep., ITU, 2008.

[4] J. G. Andrews, R. K. Ganti, M. Haenggi, N. Jindal, and S. Weber, "A primer on spatial modeling and analysis in wireless networks," IEEE Communications Magazine, vol. 48, pp. 156-163, November 2010.

[5] A. Damnjanovic, J. Montojo, Y. Wei, T. Ji, T. Luo, M. Vajapeyam, T. Yoo, O. Song, and D. Malladi, "A survey on 3GPP heterogeneous networks," IEEE Wireless Communications, vol. 18, pp. 10-21, June 2011.

[6] Z. Hasan, H. Boostanimehr, and V. K. Bhargava, "Green cellular networks: A survey, some research issues and challenges," IEEE Communications Surveys and Tutorials, vol. 13, pp. 524-540, November 2011.

[7] Y. Chen, S. Zhang, S. Xu, and G. Y. Li, "Fundamental trade-offs on green wireless networks," IEEE Communications Magazine, vol. 49, pp. 30-37, June 2011.

[8] D. Gonzalez G., H. Yanikomeroglu, M. Garcia-Lozano, and S. Ruiz Boque, "A novel multi-objective framework for cell switch-off in dense cellular networks," IEEE International Conference on Communications (ICC), pp. 2641-2647, June 2014. 
[9] R. Schoenen and H. Yanikomeroglu, "User-in-the-loop: Spatial and temporal demand shaping for sustainable wireless networks," IEEE Communications Magazine, vol. 52, pp. 196-203, February 2014.

[10] V. Paxson and S. Floyd, "Wide area traffic: the failure of Poisson modeling," IEEE/ACM Transactions on Networking (ToN), vol. 3, pp. 226-244, June 1995.

[11] W. Fischer and K. Meier-Hellstern, "The Markov-Modulated Poisson Process (MMPP) cookbook," Performance Evaluation, vol. 18, no. 2, pp. 149-171, 1993.

[12] H. Heffes and D. Lucantoni, "A Markov modulated characterization of packetized voice and data traffic and related statistical multiplexer performance," IEEE Journal on Selected Areas in Communications, vol. 4, pp. 856-868, September 1986.

[13] A. Klemm, C. Lindemann, and M. Lohmann, "Modeling IP traffic using the batch Markovian arrival process," Performance Evaluation, vol. 54, pp. 149-173, October 2003.

[14] L. Muscariello, M. Mellia, M. Meo, M. Ajmone Marsan, and R. Lo Cigno, "Markov models of Internet traffic and a new hierarchical MMPP model," Computer Communications, vol. 28, pp. 1835-1851, October 2005.

[15] A. Dainotti, A. Pescapé, P. S. Rossi, F. Palmieri, and G. Ventre, "Internet traffic modeling by means of hidden Markov models," Computer Networks, vol. 52, pp. 2645-2662, October 2008.

[16] S. Maheshwari, K. Vasu, S. Mahapatra, and C. Kumar, "A joint-parametric realistic traffic model for wireless Internet using hidden Markov model," IEEE Cross Strait Quad-Regional Radio Science and Wireless Technology Conference (CSQRWC), pp. 806-811, July 2011.

[17] Y. Xie, J. Hu, Y. Xiang, S. Yu, S. Tang, and Y. Wang, "Modeling oscillation behavior of network traffic by nested hidden Markov model with variable state-duration," IEEE Transactions on Parallel and Distributed Systems, vol. 24, pp. 1807-1817, September 2013.

[18] H. ElSawy, E. Hossain, and M. Haenggi, "Stochastic geometry for modeling, analysis, and design of multi-tier and cognitive cellular wireless networks: A 
survey," IEEE Communications Surveys \& Tutorials, vol. 15, pp. 996-1019, July 2013.

[19] C. Bettstetter, M. Gyarmati, and U. Schilcher, "An inhomogeneous spatial node distribution and its stochastic properties," ACM Symposium on Modeling, Analysis, and Simulation of Wireless and Mobile Systems, pp. 400-404, June 2007.

[20] C. Qvarfordt and P. Legg, "Evaluation of LTE HetNet deployments with realistic traffic models," IEEE International Workshop on Computer Aided Modeling and Design of Communication Links and Networks (CAMAD), pp. 307-311, September 2012 .

[21] "3GPP TR 36.814: Further advancements for E-UTRA physical layer," tech. rep., 3GPP, 2010.

[22] H. Dhillon, R. Ganti, and J. Andrews, "Modeling non-uniform UE distributions in downlink cellular networks," IEEE Wireless Communications Letters, vol. 2, pp. 339-342, June 2013.

[23] M. Taranetz and M. Rupp, "Performance of femtocell access point deployments in user hot-spot scenarios," IEEE Telecommunication Networks and Applications Conference (ATNAC), November 2012.

[24] D. Lee, S. Zhou, X. Zhong, Z. Niu, X. Zhou, and H. Zhang, "Spatial modeling of the traffic density in cellular networks," IEEE Wireless Communications Magazine, vol. 21, pp. 80-88, February 2014.

[25] Z. Wang, R. Schoenen, H. Yanikomeroglu, and M. St-Hilaire, "Load balancing in cellular networks with user-in-the-loop: A spatial traffic shaping approach," IEEE International Conference on Communications (ICC), June 2015.

[26] M. Mirahsan, Z. Wang, R. Schoenen, H. Yanikomeroglu, and M. St-Hilaire, "Unified and non-parameterized statistical modeling of temporal and spatial traffic heterogeneity in wireless cellular networks," IEEE International Conference on Communications (ICC) Workshops, pp. 55-60, June 2014. 
[27] M. Mirahsan, R. Schoenen, and H. Yanikomeroglu, "Statistical modeling of spatial traffic distribution with adjustable heterogeneity and bs-correlation in wireless cellular networks," IEEE Global Communications Conference (Globecom), pp. 3647-3652, December 2014.

[28] M. Mirahsan, R. Schoenen, and H. Yanikomeroglu, "HetHetNets: Heterogeneous traffic distribution in heterogeneous wireless cellular networks," IEEE Journal on Selected Areas in Communications (JSAC) Special Issue on Heterogeneous Networks, vol. 33, pp. 2252 - 2265, October 2015.

[29] F. Yu and V. Leung, "Mobility-based predictive call admission control and bandwidth reservation in wireless cellular networks," Computer Networks, vol. 38, pp. 577-589, April 2002.

[30] E. W. Knightly and N. B. Shroff, "Admission control for statistical QoS: theory and practice," IEEE Network, vol. 13, pp. 20-29, March/April 1999.

[31] M. H. Ahmed, "Call admission control in wireless networks: A comprehensive survey," IEEE Communications Surveys and Tutorials, vol. 7, pp. 50-69, March 2005.

[32] M. Ghaderi and R. Boutaba, "Call admission control in mobile cellular networks: a comprehensive survey," Wireless Communications and Mobile Computing, vol. 6, pp. 69-93, August 2006.

[33] Y. Fang and Y. Zhang, "Call admission control schemes and performance analysis in wireless mobile networks," IEEE Transactions on Vehicular Technology, vol. 51, pp. 371-382, March 2002.

[34] N. M. K. Chowdhury and R. Boutaba, "Network virtualization: state of the art and research challenges," IEEE Communications Magazine, vol. 47, pp. 20-26, July 2009.

[35] N. M. K. Chowdhury and R. Boutaba, "A survey of network virtualization," Computer Networks, vol. 54, pp. 862-876, April 2010.

[36] R. Jain and S. Paul, "Network virtualization and software defined networking for cloud computing: a survey," IEEE Communications Magazine, vol. 51, no. 11, pp. $24-31,2013$. 
[37] N. M. K. Chowdhury, M. R. Rahman, and R. Boutaba, "Virtual network embedding with coordinated node and link mapping," IEEE INFOCOM 2009, pp. 783791, 2009 .

[38] M. Chowdhury, M. R. Rahman, and R. Boutaba, "Vineyard: Virtual network embedding algorithms with coordinated node and link mapping," IEEE/ACM Transactions on Networking (TON), vol. 20, pp. 206-219, February 2012.

[39] M. Yu, Y. Yi, J. Rexford, and M. Chiang, "Rethinking virtual network embedding: substrate support for path splitting and migration," ACM SIGCOMM Computer Communication Review, vol. 38, pp. 17-29, April 2008.

[40] X. Cheng, S. Su, Z. Zhang, H. Wang, F. Yang, Y. Luo, and J. Wang, "Virtual network embedding through topology-aware node ranking," ACM SIGCOMM Computer Communication Review, vol. 41, pp. 38-47, April 2011.

[41] K.-M. Park and C.-K. Kim, "A framework for virtual network embedding in wireless networks," ACM Future Internet Technologies, pp. 5-7, June 2009.

[42] D. Yun and Y. Yi, "Virtual network embedding in wireless multihop networks," ACM Future Internet Technologies, pp. 30-33, June 2011.

[43] M. Yang, Y. Li, L. Zeng, D. Jin, and L. Su, "Karnaugh-map like online embedding algorithm of wireless virtualization," 15th International Symposium on Wireless Personal Multimedia Communications (WPMC) 2012, pp. 594-598, 2012.

[44] M. Yang, Y. Li, D. Jin, J. Yuan, L. Su, and L. Zeng, "Opportunistic spectrum sharing based resource allocation for wireless virtualization," Seventh International Conference on Innovative Mobile and Internet Services in Ubiquitous Computing (IMIS) 2013, pp. 51-58, 2013.

[45] M. Yang, Y. Li, J. Liu, D. Jin, J. Yuan, and L. Zeng, "Opportunistic spectrum sharing for wireless virtualization," IEEE Wireless Communications and Networking Conference (WCNC) 2014, pp. 1803-1808, 2014.

[46] J. van de Belt, H. Ahmadi, and L. E. Doyle, "A dynamic embedding algorithm for wireless network virtualization," IEEE 80th Vehicular Technology Conference (VTC Fall) 2014, pp. 1-6, 2014. 
[47] M. Mirahsan, R. Schoenen, H. Yanikomeroglu, G. Senarath, and N. Dung-Dao, "User-in-the-loop for hethetnets with backhaul capacity constraints," IEEE Wireless Communications, vol. 22, pp. 50-57, October 2015.

[48] M. Mirahsan, H. Yanikomeroglu, G. Senarath, and N. Dao, "Analytic modeling of sir in cellular networks with heterogeneous traffic," IEEE Communications Letters, vol. 20, pp. 1627-1630, May 2016.

[49] M. Mirahsan, H. Farmanbar, and H. Yanikomeroglu, "Joint backhaul and access optimization for service-segment-based vn admission control," IEEE International Conference on Communications (ICC), vol. 5, no. 1, pp. 97-101, 2017.

[50] M. Mirahsan, R. Schoenen, S. Szyszkowicz, and H. Yanikomeroglu, "Spatial heterogeneity of users in wireless cellular networks based on open urban maps," IEEE International Conference on Communications (ICC), June 2015.

[51] M. Mirahsan, N. Senarath, N. Dao, and H. Farmanbar, "Method and apparatus for admission control of virtual networks in a backhaul-limited communication network," Apr. 13 2017. US Patent App. 15/181,913.

[52] N. Senarath, N. DAO, P. Leroux, and M. Mirahsan, "Methods and systems for provisioning a virtual network in software defined networks," Apr. 14 2016. US Patent App. 14/877,307.

[53] A. T. Andersen and B. F. Nielsen, "A Markovian approach for modeling packet traffic with long-range dependence," IEEE Journal on Selected Areas in Communications, vol. 16, pp. 719-732, August 1998.

[54] G. Casale, E. Z. Zhang, and E. Smirni, Interarrival Times Characterization and Fitting for Markovian Traffic Analysis. Internat. Begegnungs-und Forschungszentrum für Informatik, 2008.

[55] "OpenStreetMap." http: //www. openstreetmap.org/.

[56] J. Rataj, I. Saxl, and K. Pelikán, "Convergence of randomly oscillating point patterns to the Poisson point process," Applications of Mathematics, vol. 38, no. 3, pp. 221-235, 1993. 
[57] V. Lucarini, "From symmetry breaking to Poisson point process in 2d Voronoi tessellations: The generic nature of hexagons," Journal of Statistical Physics, vol. 130, pp. 1047-1062, March 2008.

[58] B. Błaszczyszyn and D. Yogeshwaran, "On comparison of clustering properties of point processes," Advances in Applied Probability, vol. 46, pp. 1-20, January 2014.

[59] P. J. Clark and F. C. Evans, "Distance to nearest neighbor as a measure of spatial relationships in populations," Ecology, vol. 35, no. 4, pp. 445-453, 1954.

[60] C. D. Barr, Applications of Voronoi Tessellations in Point Pattern Analysis. ProQuest, 2008.

[61] J. Møller and D. Stoyan, "Stochastic geometry and random tessellations," tech. rep., Department of Mathematical Sciences, Aalborg University, 2007.

[62] L. Sirovich, F. Cavallini, F. Pettenati, and M. Bobbio, "Natural-neighbor isoseismals," Bulletin of the Seismological Society of America, vol. 92, no. 5, pp. 19331940, 2002.

[63] J. Møller and R. P. Waagepetersen, "Modern statistics for spatial point processes," Scandinavian Journal of Statistics, vol. 34, pp. 643-684, December 2007.

[64] E. Gilbert, "Random subdivisions of space into crystals," The Annals of Mathematical Statistics, vol. 33, no. 3, pp. 958-972, 1962.

[65] K. Borovkov and D. Odell, "Simulation studies of some voronoi point processes," Acta Applicandae Mathematicae, vol. 96, pp. 87-97, May 2007.

[66] M. Tanemura, "Statistical distributions of Poisson Voronoi cells in two and three dimensions," Forma-Tokyo, vol. 18, no. 4, pp. 221-247, 2003.

[67] L. Muche, "Distributional properties of the three-dimensional Poisson Delaunay cell," Journal of Statistical Physics, vol. 84, pp. 147-167, July 1996.

[68] P. Rathie, "On the volume distribution of the typical Poisson-Delaunay cell," Journal of Applied Probability, pp. 740-744, 1992. 
[69] R. Miles, "The random division of space," Advances in Applied Probability, pp. 243-266, 1972.

[70] A. Leon-Garcia, Probability and Random Processes for Electrical Engineering. Reading, Massachusetts: Addison-Wesley Publishing Company, Inc, 1994.

[71] J. Monserrat, P. Sroka, G. Auer, J. Cabrejas, D. Martin, A. Mihovska, R. Rossi, A. Saul, and R. Schoenen, "Advanced radio resource management for IMTAdvanced in WINNER+ (II)," ICT-MobileSummit, June 2010.

[72] D. Bültmann, T. Andre, and R. Schoenen, "Analysis of 3GPP LTE-Advanced cell spectral efficiency," Personal, Indoor, and Mobile Radio Communications (PIMRC) 2010, September 2010.

[73] R. Schoenen, A. Otyakmaz, and Z. Xu, "Resource allocation and scheduling in FDD multihop cellular systems," International Workshop on Multiple Access Communications (MACOM) at IEEE ICC 2009, June 2009.

[74] Wikipedia, "Exponentially modified Gaussian distribution — wikipedia, the free encyclopedia," 2015. [Online; accessed 6-June-2015].

[75] D. Moltchanov, "Distance distributions in random networks," Elsevier Ad Hoc Networks, vol. 10, pp. 1146-1166, August 2012.

[76] X. Ge, H. Cheng, M. Guizani, and H. Tao, "5G wireless backhaul networks: challenges and research advances," IEEE Network, vol. 28, pp. 6-11, November 2014.

[77] T. Bai and R. W. Heath, "Location-specific coverage in heterogeneous networks," IEEE Signal Processing Letters, vol. 20, no. 9, pp. 873-876, 2013.

[78] H. Tabassum, Z. Dawy, E. Hossain, and M.-S. Alouini, "Interference statistics and capacity analysis for uplink transmission in two-tier small cell networks: A geometric probability approach," IEEE Transactions on Wireless Communications, vol. 13, no. 7, pp. 3837-3852, 2014.

[79] R. Schoenen, G. Bulu, A. Mirtaheri, T. Beitelmal, and H. Yanikomeroglu, "First survey results of quantified user behavior in user-in-the-loop scenarios for sustainable wireless networks," IEEE VTC Fall-2012, pp. 1-5, September 2012. 
[80] R. Schoenen, G. Bulu, A. Mirtaheri, T. Beitelmal, and H. Yanikomeroglu, "Quantified user behavior in user-in-the-loop spatially and demand controlled cellular systems," European Wireless Conference, pp. 1-8, April 2012.

[81] M. Rahman and H. Yanikomeroglu, "Enhancing cell-edge performance: a downlink dynamic interference avoidance scheme with inter-cell coordination," IEEE Transactions on Wireless Communications, vol. 9, pp. 1414-1425, April 2010.

[82] H. Kim, G. De Veciana, X. Yang, and M. Venkatachalam, "Distributed-optimal user association and cell load balancing in wireless networks," IEEE/ACM Transactions on Networking, vol. 20, pp. 177-190, February 2012.

[83] Q. Ye, B. Rong, Y. Chen, M. Al-Shalash, C. Caramanis, and J. G. Andrews, "User association for load balancing in heterogeneous cellular networks," IEEE Transactions on Wireless Communications, vol. 12, pp. 2706-2716, June 2013.

[84] J. G. Andrews, S. Singh, Q. Ye, X. Lin, and H. S. Dhillon, "An overview of load balancing in hetnets: Old myths and open problems," IEEE Wireless Communications, vol. 21, pp. 18-25, April 2014.

[85] P. J. Burke, "The output of a queuing system," Operations Research, vol. 4, no. 6, pp. 699-704, 1956.

[86] D. Gross and C. Harris, Fundamentals of Queueing Theory. John Wiley and Sons, 1998.

[87] S. Boyd and L. Vandenberghe, Convex Optimization. Cambridge university press, 2004.

[88] Wikipedia, "Poisson distribution — wikipedia, the free encyclopedia," 2015. [Online; accessed 8-June-2015].

[89] 3rd Generation Partnership Project; Technical Specification Group Radio Access Network, "Evolved Universal Terrestrial Radio Access (E-UTRA); Further advancements for E-UTRA physical layer aspects (Release 9)," tech. rep., 3GPP TR 36.814, 2010.

[90] B. Błaszczyszyn and D. Yogeshwaran, "On comparison of clustering properties of point processes," Advances in Applied Probability, vol. 46, no. 1, pp. 1-20, 2014. 
[91] L. R. Ford Jr and D. R. Fulkerson, "A suggested computation for maximal multicommodity network flows," Management Science, vol. 5, no. 1, pp. 97-101, 1958. 


\section{Appendix A}

\section{Proof of the Simplest Polynomial Potential Function}

First, note that every BS Voronoi cell can be divided into sub-triangles which are each comprised of the BS as a vertex and the lines connecting the BS to the edge. An example is shown in Fig. 43. Increasing the number of sub-triangles to large numbers assures that the whole area is covered.

Also note that for macro-BSs, some parts of the Voronoi cell might be covered by pico-cells. However, since the sum of potential value inside each pico-cell is 0 and the pico-BSs are distributed uniformly, it does not affect the sum potential values in macro-cells.

If the rules of potential function hold inside each sub-triangle, we can infer that they hold for entire Voronoi cell because the potential value of each cell is simply the sum of potential values of sub-triangles. Therefore, the required condition for potential function is as follows:

- $P(x, y)=+1$ for cell center points,

- $P(x, y)=-1$ for Voronoi cell edge points,

- $\iint_{\Delta_{i}} P(x, y) d x d y=0, \quad \forall i$,

where $\Delta_{i}$ are the sub-triangles.

Since the potential value is assumed to be constant on each line parallel to the cell edges (as shown in Fig. 44), it means that the last condition can be written as follows:

$$
\int_{x=0}^{X} \int_{y=0}^{c x} P(x, y) d x d y=\int_{x=0}^{X} c x P(x) d x
$$




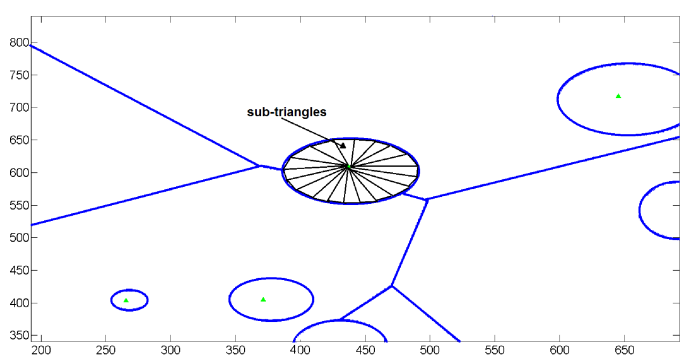

Figure 43: Every BS Voronoi cell can be divided into sub-triangles which are each comprised of the BS as a vertex and the lines connecting the BS to the edge.

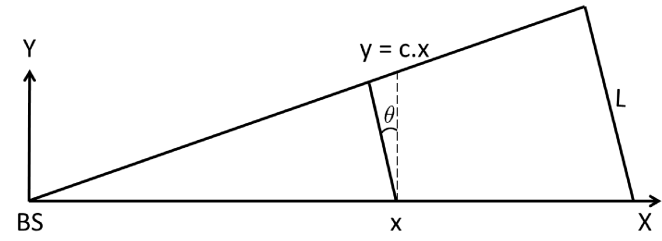

Figure 44: The potential value is assumed to be constant on each line parallel to the cell edges. So, the $\mathrm{P}$ value is only a function of $x$.

where constant $c$ is

$$
c=\frac{L}{X \cos \theta},
$$

and $L$ is the length of Voronoi cell edge and $\theta$ is the angle associated with the BS vertex. Increasing the number of sub-triangles, $\theta$ tends to 0 and $\cos \theta$ tends to 1 . So,

$$
c \simeq \frac{L}{X}
$$

Now, assume that $\mathrm{P}$ is a polynomial function. With substitution of $\mathrm{P}$ function in the conditions, we can see that $\mathrm{P}$ cannot have a degree of one. So, the minimum degree for $\mathrm{P}$ is two. With substitution of a second-degree polynomial function in the potential function conditions, the $\mathrm{P}$ function is calculated as

$$
\frac{-2 x^{2}}{X^{2}}+1
$$

which can be generalized to any arbitrary point $(x, y)$. This proves that the simplest polynomial $\mathrm{P}$ function is

$$
P(x, y)=\frac{-2(d(x, y))^{2}}{(D(x, y))^{2}}+1
$$




\section{Appendix B}

\section{The Movement of the UEs to the New Locations}

All parameters in Figure 18 except $R_{1}^{\prime}, R_{2}^{\prime}$ and $O_{2}$ are known. We define

$$
A=\left(\frac{R_{1}}{R_{2}}\right)^{n}
$$

Then, equation (21) can be stated as

$$
R_{1}^{\prime}=A R_{2}^{\prime}
$$

In Fig. 18, $D$ can be calculated as follows:

$$
D=R_{1}^{\prime} \cos \left(O_{1}\right)+R_{2}^{\prime} \cos \left(O_{2}\right)
$$

On the other hand, based on the "Law of Sines", we have

$$
\frac{\sin \left(O_{1}\right)}{\sin \left(O_{2}\right)}=\frac{R_{2}^{\prime}}{R_{1}^{\prime}}
$$

Therefore, combining (102) and (103), D is stated as

$$
D=R_{1}^{\prime} \cos \left(O_{1}\right)+R_{2}^{\prime} \sqrt{1-\sin ^{2}\left(O_{2}\right)}=R_{1}^{\prime} \cos \left(O_{1}\right)+R_{2}^{\prime} \sqrt{1-\left(\frac{R_{1}^{\prime}}{R_{2}^{\prime}} \sin \left(O_{1}\right)\right)^{2}}
$$


From (101) and (104), we obtain the following:

$$
R_{1}^{\prime}=\frac{D}{\cos \left(O_{1}\right)+\sqrt{A^{-2}-\sin ^{2}\left(O_{1}\right)}} .
$$




\section{Appendix $\mathrm{C}$}

\section{Proof of Lemma 2}

The joint pdf of $R_{1}$ and $R_{2}$ is given in [75] (30) as follows:

$$
f_{R_{1}, R_{2}}\left(r_{1}, r_{2}\right)=e^{-\lambda \pi r_{2}^{2}}(2 \lambda \pi)^{2} r_{1} r_{2}
$$

Using this density, the CDF of $R$ can be expressed as

$$
F_{R}(r)=p(R \leqslant r)=p\left(\frac{R_{1}}{R_{2}} \leqslant r\right)=p\left(\frac{R_{2}}{R_{1}} \geqslant r^{-1}\right)
$$

which can be calculated as

$$
F_{R}(r)=\int_{r_{2}=0}^{\infty} \int_{r_{1}=0}^{r r_{2}} f_{R_{1}, R_{2}}\left(r_{1}, r_{2}\right) d r_{1} d r_{2}=r^{2}
$$

this completes the proof 